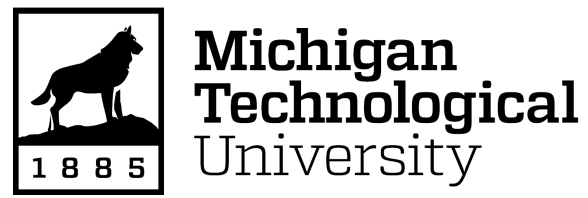

Michigan Technological University Digital Commons @ Michigan Tech

Dissertations, Master's Theses and Master's Reports

2017

COMPUTATIONAL ULTRASOUND ELASTOGRAPHY: A FEASIBILITY STUDY

Yu Wang

Michigan Technological University, ywang27@mtu.edu

Copyright 2017 Yu Wang

Recommended Citation

Wang, Yu, "COMPUTATIONAL ULTRASOUND ELASTOGRAPHY: A FEASIBILITY STUDY", Open Access

Dissertation, Michigan Technological University, 2017.

https://doi.org/10.37099/mtu.dc.etdr/389

Follow this and additional works at: https://digitalcommons.mtu.edu/etdr

Part of the Bioimaging and Biomedical Optics Commons 


\title{
COMPUTATIONAL ULTRASOUND
}

\section{ELASTOGRAPHY: A FEASIBILITY STUDY}

\author{
By \\ Yu Wang \\ A DISSERTATION \\ Submitted in partial fulfillment of the requirements for the degree of \\ DOCTOR OF PHILOSOPHY \\ In Biomedical Engineering
}

MICHIGAN TECHNOLOGICAL UNIVERSITY

2017

(C) $2017 \mathrm{Yu}$ Wang 
This dissertation has been approved in partial fulfillment of the requirements for the Degree of DOCTOR OF PHILOSOPHY in Biomedical Engineering.

Department of Biomedical Engineering

Dissertation Advisor: Jingfeng Jiang

Committee Member: Sean J. Kirkpatrick

Committee Member: Jinshan Tang

Committee Member: Min Wang

Department Chair: Sean J. Kirkpatrick 


\section{Content}

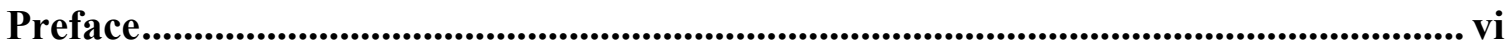

Acknowledgements .................................................................................................................. viii

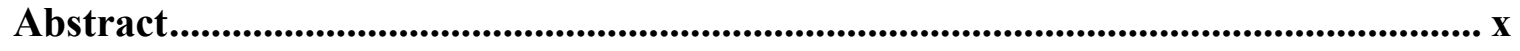

Chapter 1. Introduction ................................................................................. 1

1.1 Background of Ultrasound Elastography ....................................................... 1

1.2 Applications and Existing Problems .................................................................. 2

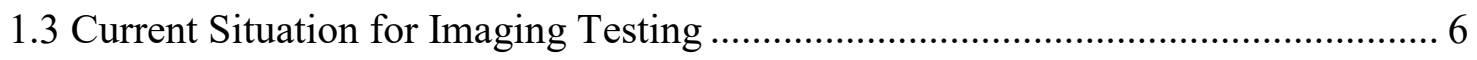

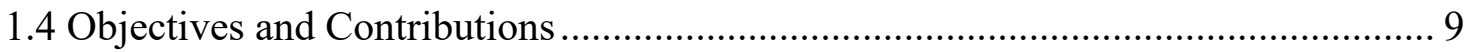

Chapter 2. Virtual Simulation Platform for Quasi-static Breast Ultrasound

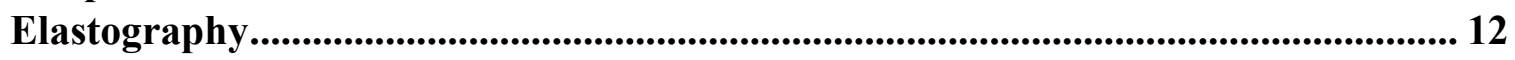

2.1 Workflow of The Proposed Simulation Platform ................................................ 12

2.2 Simulated B-mode Images Based On Complex Patterns In Heterogeneous Media 23

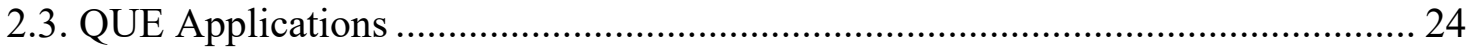

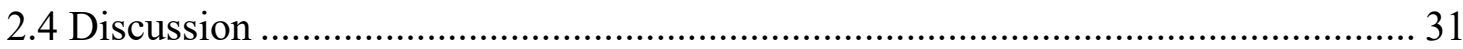

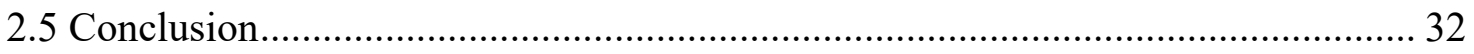

Chapter 3. Virtual Simulation Platform for Acoustic Radiation Force-based Breast Elastography ............................................................................................................. 34

3.1 Workflow of Virtual Simulation Platform for Acoustic Radiation Force-based UE

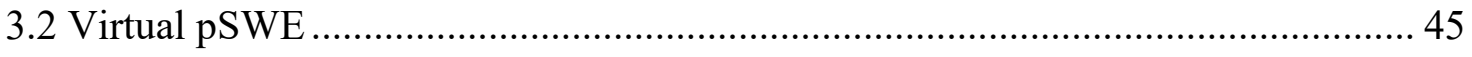

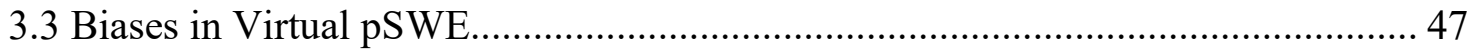

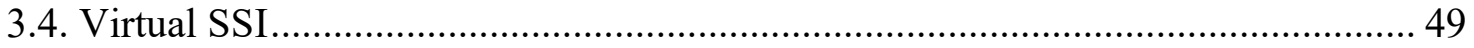

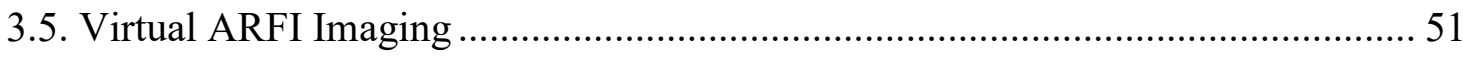

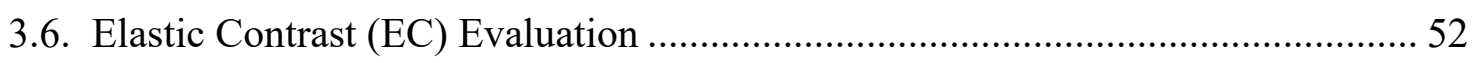

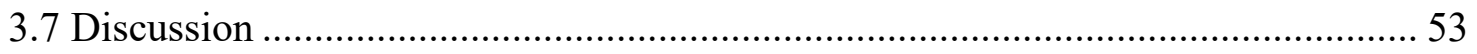

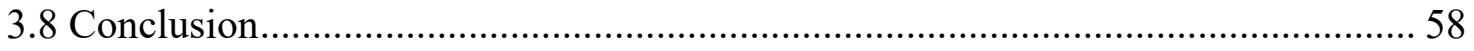

Chapter 4. Statistical analysis of SWE measurements in random two-phase media 59

4.1 Derivation of Probability Density Function (PDF) .......................................... 59

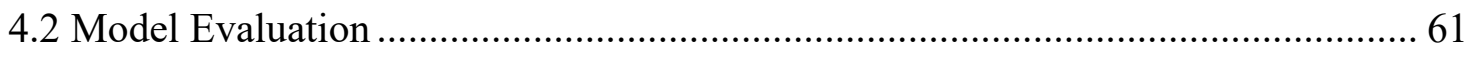

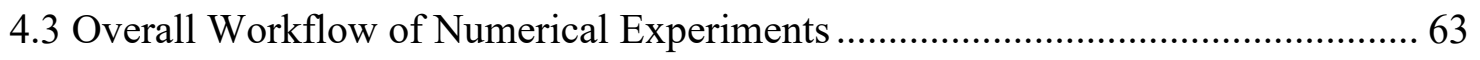




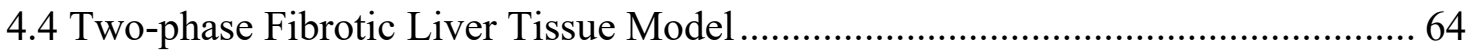

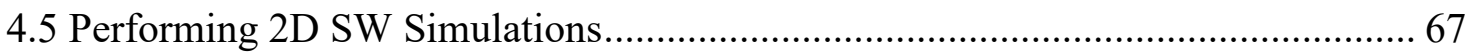

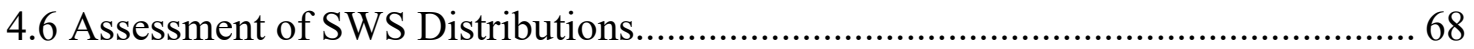

4.7 Performance of The Derived PDF.................................................................... 70

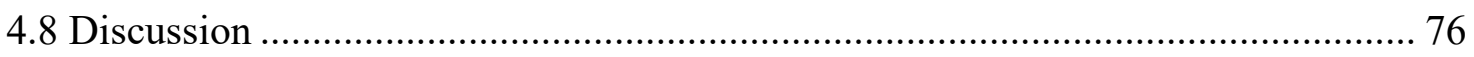

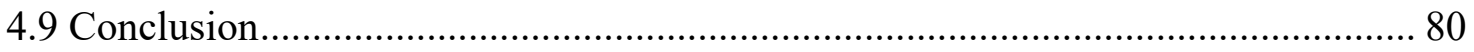

Chapter 5. Influence of Tissue Microstructures on Shear Wave Speed Measurements in Transient Elastography............................................................... 81

5.1 2D Random Two-phase Models of Fibrotic Liver Tissues .................................. 81

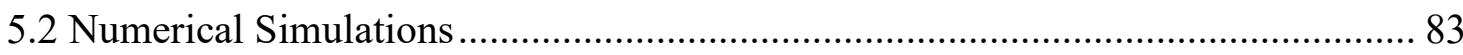

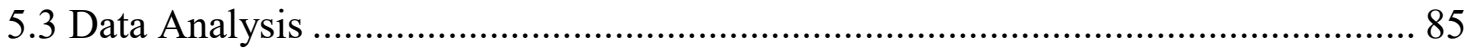

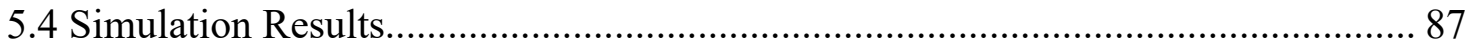

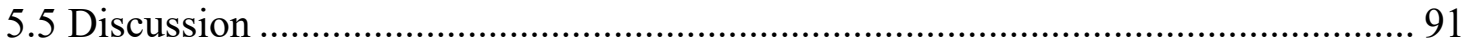

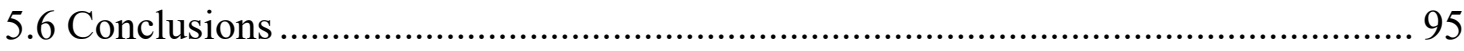

Chapter 6. A Two-dimensional (2D) Systems Biology-based Micromechanical Liver Tissue Model: A Simulation Study with Implications for Ultrasound Elastography of Liver Fibrosis ................................................................................................... 96

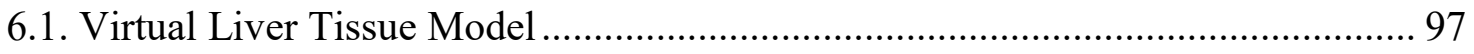

6.2. Virtual Tissue Model for Simulations of Ultrasound Compressional/Shear Waves

6.3. Validations Using Available Clinical Data ..................................................... 104

6.4. Parameters Involving in Systems Biology Simulations ................................... 105

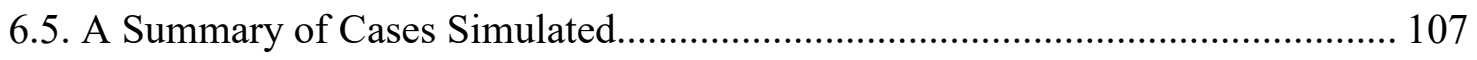

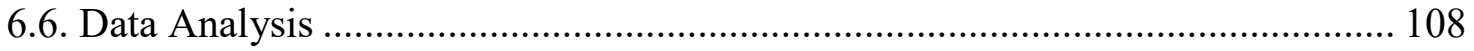

6.7. Qualitative Comparisons to Histological Characteristics (Cases 1-4) ................ 108

6.8. Parameters Analysis Using Stepwise Regression (Cases 5-6) ............................ 110

6.9. Comparisons to Wave Speed Measurements (Cases 7-9) .................................. 112

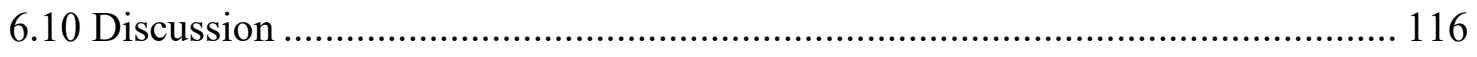

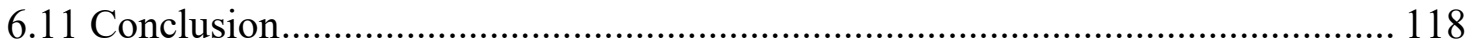

Chapter 7. Summary and Future Work ......................................................................... 119

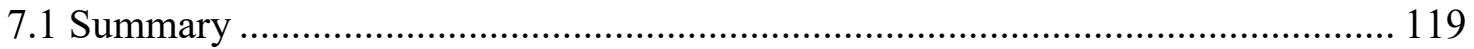

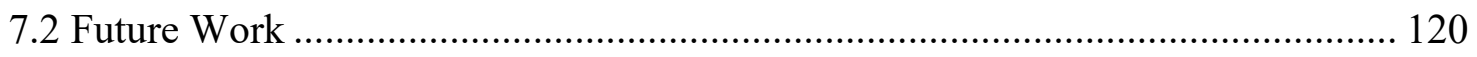




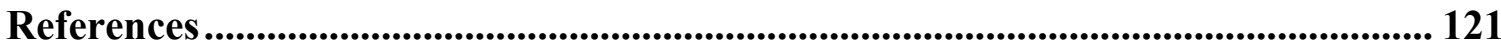

Appendix A: Geometry Creation ............................................................................... 133

Appendix B: Tissue Mechanical Models Used for QUE Simulations ........................ 136

Appendix C: Derivation of PDF for SWS Distribution in Random Multiphase Media 138

Appendix D: A Brief Description of K-Wave Simulations............................................. 142

Appendix E: A Description of Systems Biology Simulations ....................................... 145

Appendix F: Copyright Permission of Content in Chapter 2 ..................................... 148

Appendix G: Copyright Permission of Content in Chapter 3 and 4 .......................... 149 


\section{Preface}

This dissertation presents my research work in pursuing the Ph.D. degree in Biomedical Engineering at Michigan Tech University. This dissertation includes previously published or submitted papers in Chapters 2-6.

Chapter 2 contains one paper published in Medical Physics (2015 Sep; 42(9): 545366) with Emily Helminen and Dr. Jingfeng Jiang. Refer Appendix F for granted permission to be republished. As the first author, I mainly contributed on performing simulations, data analysis, and manuscript revision. Ms. Emily Helminen was an undergraduate research assistant who performed image segmentation. Dr. Jingfeng Jiang was instrumental in the idea construction and participated in the model generation and data interpretation. All authors involved in the manuscript preparation, editing and proof-reading.

Chapter 3 contains one paper published in Physics in Medicine and Biology (2017 Feb; 62(5): 1949-1969) with Dr. Bo Peng and Dr. Jingfeng Jiang. Refer Appendix G for granted permission to be republished. As the first author, I mainly contributed on performing simulations and data analysis. Dr. Peng involved in motion tracking for displacement estimations of ultrasound data. Dr. Jingfeng Jiang participated in study design and data interpretation. All authors involved in the manuscript preparation, editing and proof-reading.

Chapter 4 contains one paper published in Physics in Medicine and Biology (2017 Jan; 62(3): 1149-1171) with Dr. Min Wang and Dr. Jingfeng Jiang. Refer Appendix G for granted permission to be republished. As the first author of the paper published in Physics in Medicine and Biology, I did theoretically derivation, performed simulation and data 
analysis. Dr. Wang contributed to derivation and statistical analysis. Dr. Jingfeng Jiang oversaw the study design and participated in data interpretation. All authors involved in the manuscript preparation, editing and proof-reading.

Chapter 5 contains one paper submitted to Ultrasonic Imaging with Dr. Jingfeng Jiang. In this manuscript (under review for Ultrasonic Imaging), I performed simulations and data analysis and Dr. Jingfeng Jiang constructed the study design and performed data analysis. Both authors involved in manuscript preparation, editing and proof-reading.

Chapter 6 contains a manuscript that will be submitted to Ultrasound in Medicine and Biology. As the first author, I performed simulations, programming in SPARK, and data analysis and Dr. Jingfeng Jiang constructed the study design and performed data analysis. Both authors involved in the manuscript preparation, editing and proof-reading. 


\section{Acknowledgements}

My sincere acknowledgment should first be given to my advisor, Dr. Jingfeng Jiang, who serves not only as the academic supervisor for my Ph.D. thesis but also as a mentor for my future career. During the valuable time, I spent with Dr. Jingfeng Jiang in the past four years, he enlightened me to the interesting and mystical world of biomedical engineering, taught me essential knowledge and tools which will also play an important part in my future work, and most importantly, encouraged me for independent and critical thinking. Impacted by his persistence and inspiration, instead of easily giving up, I learned to insist on finding solutions when I encountered challenging problems. Such a great experience is invaluable for my life.

I would like to give my deep gratitude to Dr. Bo Peng, who spared no pains to assist me with complex code development and selflessly share every single detail of his experience in coding. Apart from the technique supports and fruitful discussions offered by Dr. Bo Peng, encouragement from his acted as healing walked through my hard times and made me optimistic when feeling depressed.

I would like to thank Dr. Sean J Kirkpatrick, Dr. Jinshan Tang, Dr. Min Wang for serving as my committee members and for helpful suggestions on my research.

Happy times with all my lab mates and friends in the beautiful town Houghton is always memorable. They are Kevin Sunderland, David Rosen, Jie Zhou, Liwei Geng, Weilue He, Fengde Ma, Zhiyong Yin and others not fully listed here. 
Most importantly, I would like to express my deepest gratitude to my parents Mr. Dongping Wang and Mrs. Xiantao Zhou for their love, support, and understanding. I know they did lots of things for me more than I could imagine, and such a strong family bond is my motivation to move on. Lastly, I would like to give my special thanks to my wife, Xueling Li, for her unconditional support, constant companion, and meticulous care in those years.

My research was supported in part by a research grant from the National Institutes of Health (R15CA179409), the FINISHING fellowship from the Graduate School of Michigan Technological University and the start-up fund from the Biomedical Engineering Department of Michigan Technological University. The Superior HPC provided computing time that enabled a part of this research and excellent technical supports from Dr. Gowtham are also highly appreciated. 


\begin{abstract}
Ultrasound Elastography (UE) is an emerging set of imaging modalities used to assess the biomechanical properties of soft tissues. UE has been applied to numerous clinical applications. Particularly, results from clinical trials of UE in breast lesion differentiation and staging liver fibrosis indicated that there was a lack of confidence in UE measurements or image interpretation. Confidence on UE measurements interpretation is critically important for improving the clinical utility of UE. The primary objective of my thesis is to develop a computational simulation platform based on open-source software packages including Field II, VTK, FEBio and Tetgen. The proposed virtual simulation platform can be used to simulate SE and acoustic radiation force based SWE simulations, including pSWE, SSI and ARFI.
\end{abstract}

To demonstrate its usefulness, in this thesis, examples for breast cancer detections were provided. The simulated results can reproduce what has been reported in the literature.

To statistically analyze the intrinsic variations of shear wave speed (SWS) in the fibrotic liver tissues, a probability density function (PDF) of the SWS distribution in conjunction with a lossless stochastic tissue model was derived using the principle of Maximum Entropy (ME). The performance of the proposed PDF was evaluated using Monte-Carlo (MC) simulated shear wave data and against three other commonly used PDFs. We theoretically demonstrated that SWS measurements follow a non-Gaussian distribution for the first time. One advantage of the proposed PDF is its physically meaningful parameters. Also, we conducted a case study of the relationship between shear 
wave measurements and the microstructure of fibrotic liver tissues. Three different virtual tissue models were used to represent underlying microstructures of fibrotic liver tissues.

Furthermore, another innovation of this thesis is the inclusion of "biologicallyrelevant" fibrotic liver tissue models for simulation of shear wave elastography. To link tissue structure, composition and architecture to the ultrasound measurements directly, a “biologically relevant” tissue model was established using Systems Biology. Our initial results demonstrated that the simulated virtual liver tissues qualitatively could reproduce histological results and wave speed measurements.

In conclusions, these computational tools and theoretical analysis can improve the confidence on UE image/measurements interpretation. 


\section{Chapter 1. Introduction}

\subsection{Background of Ultrasound Elastography}

Ultrasound Elastography (UE) is an emerging set of imaging modalities used to assess the biomechanical properties of soft tissues. UE can be used to measure strain, relaxation time constants, Young's modulus and shear wave speed (SWS). To the World Federation of Ultrasound in Medicine and Biology (WFUMB) [1], UE includes both strain elastography (SE) and shear wave elastography (SWE). The former is to measure quasistatic tissue response, while the latter is to measure SWS from dynamic responses of the tissue being imaged (e.g. propagation of shear wave fronts). SE [2] is a method for measuring the tissue deformation generated by applying pressure with an ultrasound transducer on the body surface. Then, the deformation can be measured (see Fig. 1.1a). The hard region strains less than the surrounding soft region. Acoustic radiation forcebased elastography [3] mainly includes two modalities: (1) monitoring the tissue response within the radiation force region of excitation (ROE) and generating images of relative differences in tissue stiffness (e.g. Acoustic Radiation Force Impulse [ARFI] imaging); and (2) monitoring SWS in the region outside ROE to quantify tissue stiffness (e.g. point shear wave elastography [pSWE], supersonic shear imaging [SSI]). In ARFI [2], the acoustic radiation force can create a localized displacement of a few microns along the ultrasound axial direction, which decays in a few milliseconds. The hard region is displaced less than the surrounding soft region (see Fig. 1.1b). In pSWE and SSI [2], the focused acoustic beam is used to generate shear waves through an acoustic radiation force impulse. Then, the ultrasonic imaging is used to monitor the resulting shear wave propagation away from 
the radiation force pushing location. The shear wave in the hard region propagates faster than that in the soft region (see Fig. 1.1c).

Soft Tissue $) \cdot($ Acoustic Radiation Force $/ \sim$ Plane Shear Wave Propagation

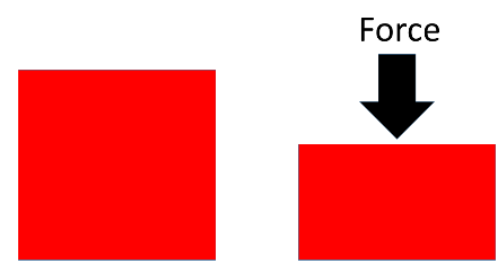

(a)

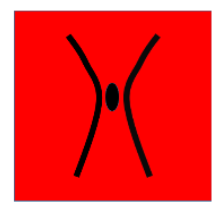

(b)

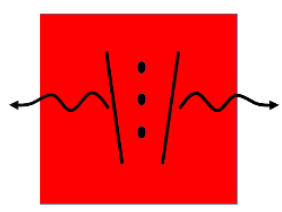

(c)

Figure 1.1 (a) Strain elastography (SE) is established by calculating the deformations caused by the ultrasound transducer. (b) The acoustic radiation force can be used to deform the tissue at the focus. In the displacement profile, the peak displacement and relaxation time can be used to obtain information about the tissue properties. (c) The acoustic radiation force is successively projected to different depths to create supersonic source. Then, the plane shear wave can be generated and propagate through the tissue [4].

\subsection{Applications and Existing Problems}

UE can be used for the investigation of many disease conditions in human organs, as disease conditions may alter underlying tissue properties. For example, UE can be used for detection and diagnosis of breast, liver, thyroid and prostate cancers [5]. To show the capability and the limitations of UE, two examples (i.e. breast cancer and liver disease) are demonstrated below.

\subsubsection{Breast Cancer Detection}

Breast cancer is known as the second leading cause of death in the female population. The American Cancer Society's (ACS) estimates that there are about 246,660 
new cases of invasive breast cancer among women in the United States in 2016 [6]. A recent comprehensive report by the ACS also confirmed that, in general, early detection is the key for decreased mortality [7]. Annual mammographic screening is often used for early detection of breast cancer, reducing mortality and morbidity, particularly in patients with tumors in fatty breast tissue [8]. However, the sensitivity and specificity are not ideal for mammography as described in the literature [9]. Breast biopsy remains the gold standard for definitive diagnosis of suspicious breast lesions. However, it is painful, exposes the patient to a risk of infection [10].

The conventional B-mode ultrasound has emerged as a useful and cost-effective modality in the workup of patients with suspected breast masses. Its traditional role has been for differentiating between solid and cystic masses and guiding biopsy procedures. However, the uncertainties in the conventional B-mode ultrasound have led to a high rate of biopsy procedures being performed [10]. Recently, ultrasound-based quasi-static elastography [11-13] (UQE) has been successfully applied to non-invasive differentiation of breast lesions $[12,14]$. However, the success of UQE is mixed. In a recent meta-analysis [15] of 22 clinical studies (up to Feb. 2011; 4713 lesions in 4,266 patients), sensitivity and specificity of the quasi-static elastography [11-13] greatly varied from $40 \%$ to $95 \%$, as compared to biopsy results. In recent years, acoustic radiation force-based elastography [16] has been successfully applied to non-invasive differentiation of breast lesions. The data in a recent meta-analysis [17] (33 clinical studies; up to Nov. 2014; 5838 lesions in 5397 patients) clearly showed that both ARFI imaging and SSI have excellent sensitivity and specificity $(85 \% \sim 90 \%)$ in terms of breast lesion characterization. Because of those 
successes, acoustic radiation force-based elastography (i.e. ARFI, pSWE, and SSI) is increasingly being employed to characterize breast masses in conjunction with the standard B-mode ultrasound. However, there are still concerns about uncertainties in acoustic radiation force-based elastography measurements. These biases were experimentally investigated [18]. In tissue mimicking materials, Zhao et al. [18] found that "SWS measurements can be transducer, depth, and lateral tracking range dependent". Another recent tissue-mimicking phantom study concluded that there was a statistically significant difference in the SWS estimation among systems and with depth into the same tissuemimicking phantom [19]. The above-referred study was authored by the Quantitative Imaging Biomarker Alliance, a research consortium under the Radiological Society of North America.

In short, early results demonstrated that there is a lack of confidence in image details of UE in the clinical community.

\subsubsection{Liver Disease Staging}

Liver fibrosis refers to a clinical condition where excessive connective tissue builds up in the liver [20]. Early stage liver fibrosis most often goes completely unnoticed, whereas late stage liver fibrosis (i.e. cirrhosis) are often fatal. The severe consequences of the progression of liver fibrosis, the common occurrence of liver fibrosis in the adult population (1-2\%), the lack of reliable methods to screen patients at high risk, and the benefit to clinical outcomes from early detection, combine to motivate the continued search for a non-invasive method which would clinically assess the stages of liver fibrosis [21]. It is worth noting that laboratory blood tests are not ready to serve as a screening tool to 
differentiate at least four liver states: fatty liver, normal liver, fibrotic liver and mixed fatty/fibrotic liver [22-25]. Measuring SWS using ultrasound-based shear wave elastography [26-28] (SWE) or transient elastography [29] (TE), has emerged as a potential clinical metric for staging liver fibrosis [30]. This is because fibrotic liver tissues are progressively hardened and the local tissue "stiffness" is often correlated to liver fibrosis stages. In the framework of ultrasound SWE, an acoustic pulse is first transmitted to produce shear waves $(\mathrm{SW})$, which propagate laterally from the direction of the acoustic pushing pulse, whereas TE uses an external mechanical vibrator to induce shear waves. Then, the shear waves can be tracked with a regular ultrasound transducer to estimate SWS, which is related to the tissue shear modulus [2]. Initial clinical successes of SWE and TE for staging liver fibrosis have been well documented [31]. The apparent potential for SWE and TE has resulted in several major vendors (e.g., General Electric, Siemens, Supersonic Imagine, Echosens, etc.) releasing commercially available packages that utilize ultrasound to measure SWS.

However, some potential problems of SWE and TE have been identified while being used for staging liver fibrosis. A study of SWE [32] found that the rate of unreliable results was high (up to 17\%). Results were determined as "unreliable" by the authors [32] if one of the following three conditions met: (1) there were fewer than 10 valid shots, (2) an interquartile range (IQR) among all results was greater than $30 \%$ and (3) a success rate was less than $60 \%$. Another study [33] reported that there was a $10 \%$ unsuccessful rate when TE was used to measure liver stiffness in children younger than 6 . Other results also 
showed that SWE may not be able to differentiate fibrosis from significant steatosis [34, 35]. Furthermore, SWS measurements could also be system-dependent [36].

In short, it is our belief that those early results indicated that more work is needed to gain confidence in clinical interpretations of SWE results.

\subsection{Current Situation for Imaging Testing}

The confidence for UE measurements or image interpretation can be gained through rigorous imaging tests with complex, heterogeneous but known media. Rigorous imaging tests are often done using human trials, tissue-mimicking phantoms or numerical tissue models, yet each testing modality has current limitations.

Human trials always associate with increasingly high costs [37]. The tissuemimicking phantoms were uniform and relatively isotropic, which are too simple to reflect the realities of complex tissue, while developments of realistic tissue-mimicking breast phantoms that can resemble the complexity of human organs and tissue properties remain as a significant challenge [38-40]. Also, these tissue-mimicking phantoms reported in the literature were still too simple. Madsen et al. [38] produced a set of five tissue-mimicking phantoms with cylindrical inclusions to assess long-term stability of geometry and elastic properties and assessing the accuracy of determination of elastic properties. Hobson et al. [39] developed two anthropomorphic uterine phantoms with sphere inclusion to assess and compare of strain imaging systems adapted for use with saline-infused sonohysterography (SIS). Carvalho et al. [40] proposed a phantom made of a mixture of water, agar, glycerin and graphite and PVC powders to mimic breast lesion with irregular edges. 
The documented numerical tissue models (Table 1.1) were still simple and didn't reflect the inherently multi-scale properties of biological tissues (i.e. large scale landmark anatomy and small scale random structures). Azar et al. [41] and Samani et al. [42] independently proposed MR-based biomechanical models to predict breast tissue deformation. An image-based breast model/phantom was created in conjunction with computed tomography data, as proposed by Hsu et al. [43]. These three models only included land mark geometries (e.g. skin, fibro-glandular tissue and fat). They didn't include small randomly distributed structures (e.g. Cooper's ligaments, milk ducts), which can increase realism and heterogeneity of the numerical model. Numerical tissue models are also used to test new algorithms in UE applications. Palmeri et al. [44] used a 3D finite element model with the uniform background to test the lateral time to peak (TTP) algorithm for SWS estimation. Park [45] designed a simple 3D numerical model embedded with a sphere (tumor) to evaluate shear modulus reconstruction algorithm. Mo et al. [46] proposed a 2D numerical model to analyze the bias of SWE measurements in thin layer tissue. However, these models were too simple to challenge these algorithms, since the realistic soft tissue is more complex than a cube embedded with a sphere or cylinder model. That is why many UE algorithms performed well and show great promise in the numerical model study but fail to live up to expectations during clinical trials.

Table 1.1. A meta-analysis of making numerical tissue models documented in literature.

\begin{tabular}{lll}
\hline \hline Authors & Numerical Model & Purpose \\
\hline Azar et al. [41], & MR-based numerical breast & Predict breast tissue \\
2001 & model, uniform model & deformation \\
\cline { 2 - 3 }
\end{tabular}


Samani et al. [47], 2001

Kallel et al. [48], 2001

Palmeri et al. [44], 2008

Wang et al. [49], 2008

Palmeri et al. [50], 2010

Lee et al. [51], 2012

Christina et al. [43], 2013

Caenen et al. [52], 2015

Park et al. [53], 2016

Mo et al. [46], 2016

Park [45], 2016

Lipman et al. [54], 2016
MR-based numerical breast Predict breast tissue model, including skin, deformation adipose, and fibro-glandular 3D finite element model, a Study contrast transfer cube embedded with a efficiency uniform cylindrical inclusion 3D finite element model Test the lateral time to peak with uniform background (TTP) algorithm 3D finite element model Study the effects of viscosity with uniform background on SWE applications 3D finite element model, a Quantify the impact of shear cube embedded with a wavelength and kernel size sphere on SWS estimation

3D finite element model, a Study shear wave cube with a stiffer cylindrical inclusion propagation through a viscoelastic medium CT-based numerical breast Generate a suite of realistic model, including fat, glandular, and skin computerized breast models from limited number of human subjects

3D finite element model Study the shear wave physics with uniform background in bounded viscoelastic medium under different material properties and acoustic force

2D finite element breast Study the elastic modulus tissue model, a rectangular contrast of the tumor to the consisting of a circular surrounding tissue under tumor different pressure in SWE 2D finite element model of Analyze the bias of SWE a thin-plate measurements in thin layer samples

3D finite element model Study the feasibility of shear with a spherical inclusion modulus reconstruction using 3D algebraic Helmholtz inversion (AHI) equation in heterogeneous media

3D finite element model Evaluate the improvement in with a spherical inclusion multidimensional directional filter in the presence of reflection artifacts 
Zhao et al. [55], 2017

2D asymmetric finite element model
Evaluate the significance of the viscoelasticity in diagnosing early-stage liver fibrosis with transient elastography

\subsection{Objectives and Contributions}

To improve the confidence in UE measurements or image interpretation for breast lesion differentiation and staging liver fibrosis, the primary objective of my thesis is to develop a computational simulation platform based on open-source software packages, including Field II (ultrasound simulator) [56], VTK (geometrical visualization and processing) [57], FEBio (finite element FE analysis) [58] and Tetgen (mesh generator) [59]. The proposed computational simulation platform can be integrated with complex, heterogeneity numerical tissue models, reflecting multi-scale properties of soft tissues.

To show the usefulness of the proposed simulation platform, UE applications of the detection of breast cancer were implemented. Both SE and SWE were simulated.

To statistically analyze the intrinsic variations of SWS in the liver fibrotic tissue, a probability density function (PDF) of the SWS distribution in stochastic tissue model was derived. The performance of the derived PDF was evaluated using Monte-Carlo (MC) simulated shear wave data against three other commonly used PDFs.

Furthermore, to relate the UE measurements to the "biologically relevant" tissue structure, composition and architecture, a virtual liver tissue with/without steatosis was created using Systems Biology method [60]. 
The rest of this dissertation is organized as follow: Chapter 2 describes the virtual breast UQE simulation platform. To show the capability of the proposed simulation platform, nonlinear and time-dependent strain imaging were simulated. Chapter 3 describes the virtual breast SWE simulation platform, which was extended from UQE simulation platform. To show the capability of the proposed simulation platform, pSWE, SSI and ARFI were simulated. Chapter 4 derives a PDF of the SWS distribution in conjunction with a lossless stochastic tissue model using the principle of maximum entropy (ME). The performance of the derived PDF was evaluated using Monte-Carlo (MC) simulated shear wave data and against three other commonly used PDFs. Chapter 5 conducted a case study to relate shear wave measurements to the microstructure of fibrotic liver tissues. Chapter 6 depicts a Systems Biology method, which can relate the UE measurements to the "biologically relevant" structures of the liver fibrosis tissue with/without steatosis. This chapter will establish the feasibility of using systems biology to construct biologically relevant tissue models linking tissue structure, composition and architecture to the ultrasound measurements directly. Chapter 7 concludes this dissertation and outlines some prospective future work.

The immediate impact of my dissertation is to build a computational framework for simulating image formation process of UE (i.e. SE and SWE), which can be used to 1) assess the confidence of quasi-static elastography measurements including strain, modulus and viscoelastic relaxation constant and 2) broaden the basic understanding of shear wave propagation in heterogeneous media. Also, my doctoral research is highly innovative: 1) the simulated ultrasound elastography images can be compared and validated by a complex 
but known media; 2) It is the first time that "biologically relevant" tissue models are used in ultrasound elastography simulations, and 3) it is also the first time that a probabilitybased assessment of SWS has been applied to SWE. 


\section{Chapter 2. Virtual Simulation Platform for Quasi-static Breast Ultrasound Elastography}

Quasi-static Ultrasound Elastography (QUE) is being used to augment in vivo characterization of breast lesions. Results from early clinical trials indicated that there was a lack of confidence in image interpretation. Such confidence can only be gained through rigorous imaging tests using complex, heterogeneous but known media. The objective of this chapter is to build a virtual breast QUE simulation platform in the public domain that can be used for rigorous imaging tests.

\subsection{Workflow of The Proposed Simulation Platform}

The use of the proposed virtual ultrasound simulation platform involves multiple components: 1) geometry creation, 2) mesh generation, 3) acoustic simulation and 4) biomechanical deformation. An illustrative example (Fig. 2.1) is provided below to demonstrate the specific steps. During the process, the starting point was an image-based geometrical model of the breast including different internal components (Section A). This geometrical model was based on "average" anatomy and represented by three-dimensional (3D) surface triangles. To simulate signals for ultrasound-based QUE applications, both pre- and post-deformed ultrasound echo signals were needed. Therefore, in addition to the un-deformed virtual breast model/phantom, a deformed breast model/phantom was also created. A simulated freehand scanning ultrasound transducer was used to deform this virtual breast model/phantom. This deforming process involved in finite element

\footnotetext{
${ }^{1}$ The material contained in this chapter was previously published in Medical physics, 2015 Sep;42(9):5453-66. Refer Appendix F for granted permission to be republished.
} 
simulations (i.e. FEBio [61]) and therefore was naturally split into mesh generation (Section B) and biomechanical simulations (Section D). Both undeformed and deformed virtual breast models/phantoms had to interact with an ultrasound simulator (i.e. Field II [62]) to generate simulated ultrasound echo signals.

The proposed virtual simulation platform leveraged existing open-source packages including Field II [62] (acoustic simulator), FEBio [61] (finite element simulator for biomechanics), VTK [63] (data visualization and processing) and Tetgen (mesh generator) [59]. The integration of these open source packages was based on a simple messagepassing scheme for the ease of use. Details of each step of this multiple-step process are provided below.

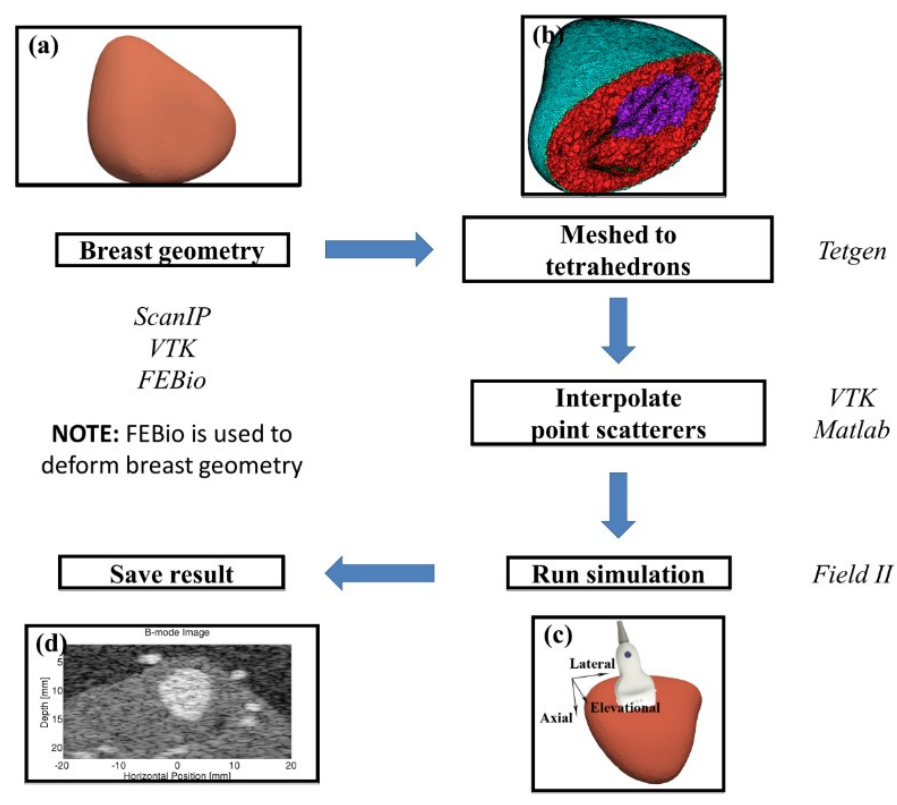

Figure 2.1. A graphic illustration of the workflow of the proposed virtual ultrasound simulation platform. The geometry of a pre- deformation breast model (a) was first created 
based on MR imaging data and, then meshed to tetrahedrons (c). In the following step, the meshed breast model/phantom was re-sampled to a rectilinear grid (d). Then acoustic parameters were assigned to the rectilinear grid for an acoustic simulator [sub-figure (e); Field II]. Based on a biomechanical finite element (FE) simulation (FEBio), the predeformation breast model/phantom can be warped to create a post-deformation breast model/phantom through interpolations. Finally, the pre- (f) and post-deformation (g) Bmode images were obtained. The blue boxes in (d) and (e) represent re-sampled the volumes of interest. Tetrahedrons (c) were converted into voxels (d) in a regular lattice grid.

\section{A. Geometry Creation}

Through image segmentation, landmark geometries such as the skin layer and fibroglandular layer were created using magnetic resonance imaging (MRI) data from a female cadaver in the Visible Human Project [64]. The in-plane resolution of these MR images was 0.33 x $0.33 \mathrm{~mm}$ with a $0.6 \mathrm{~mm}$ slice thickness. All MRI images were imported into a commercially-available ScanIP package (Simpleware Inc., Exeter, United Kingdom) [65] to perform intensity-based image segmentation to obtain the skin surface and the fibroglandular layer (Fig. 2.2a). Other computer-aided design software (e.g. Solidworks, Dassault Systems Inc., France) or image segmentation software (e.g. Open-source Vascular Modeling Toolkit[66]) can also be used to create complex geometries. To show flexibility of the proposed virtual ultrasound simulation platform, an alternate way for geometry creation and subsequent acoustic simulations (following the same procedures as described

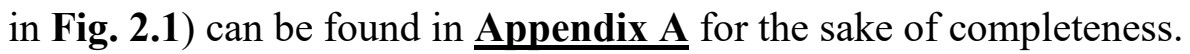

Small randomly distributed tissue structures such as milk ducts and Cooper's ligaments were added to increase the complexity and realism of the virtual breast phantom. In the Visible Human Project, MRI data were generated using torso coils and therefore 
have limited resolution. However, it would be difficult to fully resolve milk ducts and Cooper's ligaments even when breast array coils were used. Similar to the study by Mahr [67], Cooper's ligaments (blue color in Fig. 2.3) were modeled as clusters of fibers embedded into the subcutaneous fat region toward the skin layer. The breast ducts (green color in Fig. 2.3) were randomly generated by a collection of bifurcated tubes and mainly confined within the fibro-glandular region. Sizes of milk duct (diameter $1.9 \pm 0.6 \mathrm{~mm}$; see Fig. 2.2b) and Cooper's ligament (diameter 3-4 $\mathrm{mm}$ ) were based on ultrasound measurement in the literature [68]. Furthermore, intra-glandular fat (purple color in Fig. 2.3a) is modeled as small adipose compartments (elongated random ellipsoids) within the fibro-glandular region, consistent with observations from B-mode ultrasound data.

Those above-mentioned random structures were mathematically defined by their boundaries using $3 \mathrm{D}$ triangulated surfaces. They may belong to more than one anatomical region. Boolean operations [69] (i.e. vtkBooleanOperationPolyDataFilter) were used to alter existing boundaries to accommodate that. Similar Boolean operations were used when the embedded breast tumor (yellow colored object in Fig. 2.3a-d) may infiltrate out of the fibro-glandular tissue region (Fig. 2.3b), grow out of breast ducts (Fig. 2.3c) and disrupt Cooper's ligaments (Fig. 2.3d).

Python scripts derived from Visualization Toolkit [63] (VTK, Kitware Inc., NY, USA) were used to create above-mentioned small structures. Interested readers are referred to $\underline{\text { Appendix B }}$ for algorithmic details. 


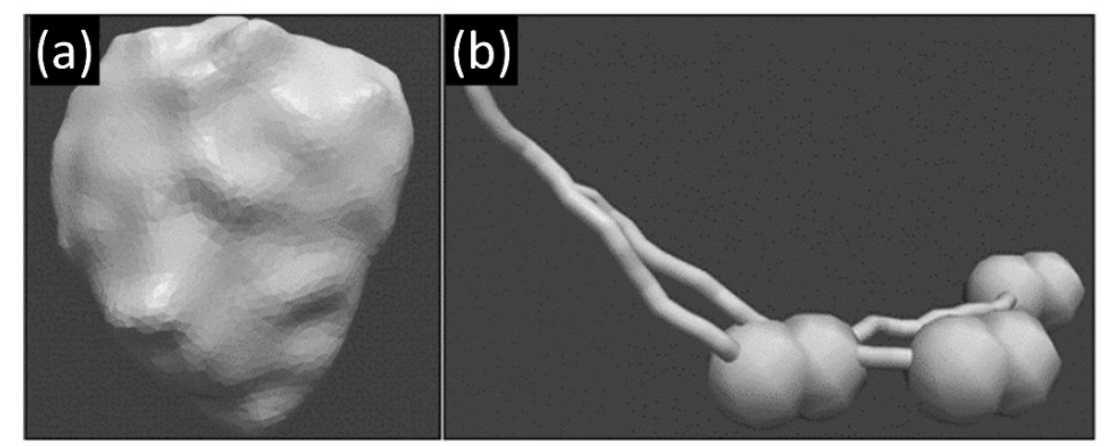

Figure 2.2. A collection of images related to the virtual breast model: (a) fibro-glandular tissue geometry segmented from MRI data; and (b) a geometry of milk ducts created using VTK [63].

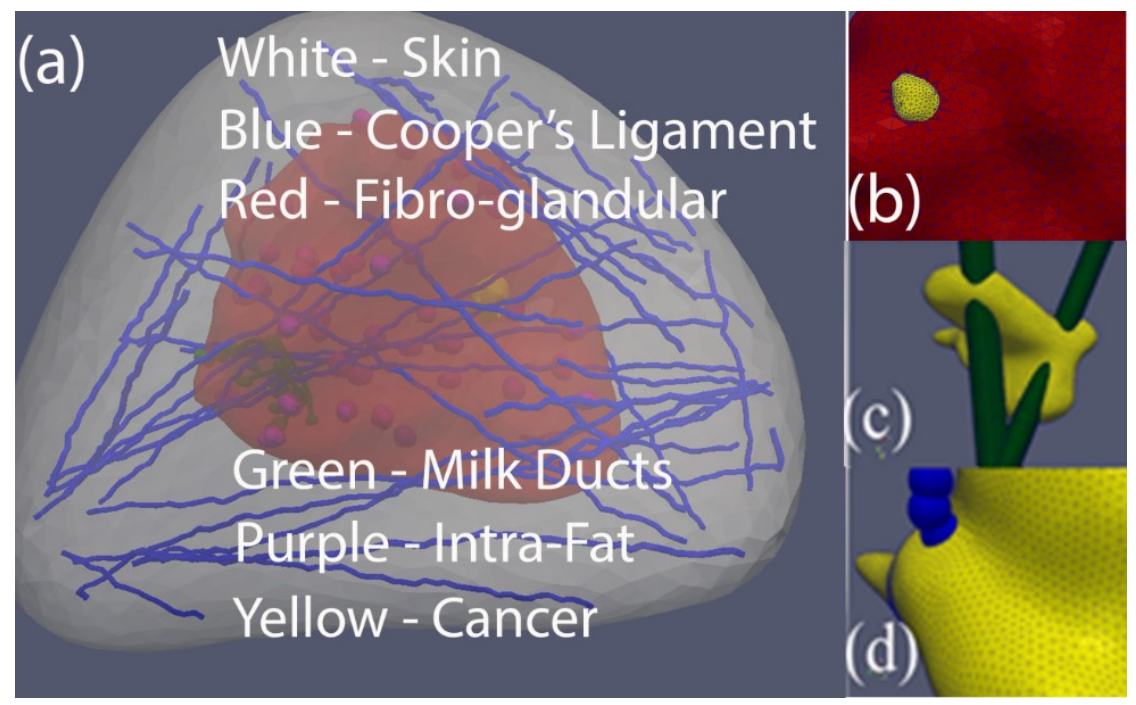

Figure 2.3. (a) An illustration of image-based breast phantom creation. Major anatomical regions are derived from $3 \mathrm{D}$ MR data, while other lower scale structures (e.g. Cooper's ligament, intra-glandular fat and milk ducts) that are significant to breast mechanics are randomly distributed structures generated by in-house software. Realism can be enhanced through geometrical Boolean operations: for example, (b) tumor invasion (out of the fibro-glandular region) to the subcutaneous fat, (c) tumor growth out of/from breast milk ducts, and (d) tumor disruption of Cooper's Ligament. In (a), the volume between the skin and fibro-glandular region is mainly occupied by breast fat. Intra-Fat denotes small adipose compartments within the fibro-glandular region. Of note, for visualization purpose, the number of random structures was intentionally reduced.

\section{B. Mesh Generation}


Once a geometric breast model (i.e. multiple [3D] complex triangulated surfaces) is obtained, an open source mesh generator Tetgen [59] (version 1.4.2; WIAS, Berlin, Germany) was used to generate high quality tetrahedral meshes as shown in Fig. 2.3b. Overall dimensions of the (irregularly shaped) breast model are approximately $62 \mathrm{~mm} \times$ $68 \mathrm{~mm} \times 41 \mathrm{~mm}$, resulting in a volume size of $62600 \mathrm{~mm}^{3}$. Tetgen uses constrained Delaunay Triangulation to partition any enclosed 3D geometry/space into tetrahedrons. Furthermore, the $3 \mathrm{D}$ enclosed breast model/phantom was also automatically divided into 6 different tissue types as shown in Table B1 in $\underline{\text { Appendix B }}$ and illustrated in Fig. 2.3. Two meshes were created. The fine and coarse meshes resulted in approximately 3.5 and 2.0 million finite (computing tetrahedral) elements, respectively.

\section{Acoustic Simulation}

In this study, our simulation platform has been integrated to Field II [62], a popular medical ultrasound simulator. In order to use the Field II [62] simulator, the meshed breast model/phantom was first re-sampled to a rectilinear $(1-\mathrm{mm} \times 1-\mathrm{mm} \times 1-\mathrm{mm})$ grid, as shown in Fig. 2.1d. Then, multiple randomly positioned point scatterers were placed in each 1$\mathrm{mm} \times 1$-mm $\times 1$-mm grid to ensure that there would be a sufficient number of point scatterers within a resolution cell. The original unstructured mesh was used to determine whether or not a point scatterer should belong to a material type. That resulted in approximately 10 scatterers $/ \mathrm{mm}^{3}$. The number of scatterers per resolution cell was varied from 10 scatterers $/ \mathrm{mm}^{3}$ to 50 scatterers $/ \mathrm{mm}^{3}$. By doing so, we verified that results of acoustic and QUE simulations were not sensitive to the proposed conversion method. 
Acoustic reflective coefficients were assigned to each point scatterer. It is worth noting that each point scatterer inherited one of six material types (see Mesh Generation Section). The reflective coefficients of 6 material types required by Field II were empirically chosen to show B-mode contrast, based on acoustic impedance data available in the literature[70]. A linear array ultrasound transducer was simulated and its relevant parameters are listed in Table 2.1 below. Perfect matching layers around the boundaries were automatically added by FIELD II to avoid undesirable wave reflections at the boundaries. Acoustic attenuation was not simulated.

Table 2.1. Parameters of the linear array ultrasound transducer used by Field II

\begin{tabular}{ll}
\hline Parameter & Field II \\
\hline Sampling frequency (MHz) & 100 \\
Center frequency (MHz) & 6 \\
Bandwidth (\%) & 50 \\
Element width (mm) & 1.5 \\
Element height (mm) & 5 \\
Spacing between elements & 0 \\
(mm) & 15 \\
Focal depth (mm) & 64 \\
Number of active elements & 256 \\
Number of scan lines & \\
\hline
\end{tabular}

\section{Biomechanical Simulation}

In order to simulate tissue deformation in QUE, an open source finite element (FE) package, Finite Elements for Biomechanics [61] (FEBio, University of Utah, UT, USA), was integrated into the proposed virtual ultrasound simulation platform. Using an in-house Python script, the tetrahedral mesh from Section C was imported into the FEBio [61] (version 1.8). Mesh sensitivity for finite element modeling was performed for two above- 
mentioned meshes (i.e. 2.2 and 3.5 million elements). The resultant displacements between those two meshes differed less than $10^{-3} \mathrm{~mm}$ for all locations investigated up to $15 \%$ of deformation. Therefore, the coarse mesh was used for all subsequent finite element simulations. The average element size of this coarse mesh was about $0.03-\mathrm{mm}^{3}$.

Two different QUE applications were simulated using the FEBio software as follows.

\section{D.1. Nonlinear QUE [71, 72] via Strain Imaging under Large Deformation}

As shown in Fig. 2.1e, a simulated ultrasound transducer was first put in contact with the skin and then used to push the breast tissue downward in the FE simulation. This boundary condition was to mimic freehand scanning in a clinical setting. More specifically, a small contact area on the [simulated] skin surface (approximately $4 \mathrm{~cm} \times 1.5 \mathrm{~cm}$ ) was selected. A range of deformation (up to $15 \%$ compression) was applied downward onto the selected contract surface. In this application, all 6 materials were assumed to be hyper elastic and follow the Veronda-Westmann model [73]. Displacements were calculated for every $1 \%$ increment of deformation.

\section{D.2. Time-dependent QUE via Strain Imaging}

In order to simulate strain/displacement creep under freehand scanning, a small compressive force of 1 Newton was applied within 1 second and the quasi-static compression was held for additional 14 seconds to calculate displacement creep using the FEBio software. The 1 Newton force was uniformly applied onto the transducer contact area (approximately $4 \mathrm{~cm} \times 1.5 \mathrm{~cm}$ ). In this application, all 6 materials were assumed to be 
a solid mixture [61] of hyper-elasticity (i.e. Veronda-Westmann model [73]) and quasilinear viscoelasticity (i.e. Kevin-Vigot Model [74]).

After the completion of above-mentioned FEBio simulations, a series of three transformations were applied to the FE-mesh and displacements in a sequence. The first transformation was a coordinate rotation of the FE-mesh and related displacements. The dominant motion/compression direction and the width direction of the simulated transducer were first set as the axial and lateral directions, respectively (i.e. $\mathrm{Y}$ and $\mathrm{X}$ axes in the Field II, respectively) for subsequent acoustic simulations. Then, a coordinate rotation was performed to map the FE-mesh coordinates to the acoustic simulation coordinates. The second transformation was simply a translation. By doing so, we made sure the center of the transducer was located at $(0,0,0)$ as required by Field II. This third transformation simply subtracted absolute displacements of the (transducer) contact surface from the already transformed FE-simulated displacements. After three transformations, all resultant displacements and coordinates were relative to the (transducer) contact surface. Consequently, this sequence of transformed displacement fields and coordinates could be readily used for acoustic simulations, as described in Section C above. Acoustic reflective indices were re-assigned to account for the mechanical deformation. More detailed information related to elastic and viscoelastic mechanical parameters using in both abovementioned applications can be found in $\underline{\text { Appendix B. }}$.

\section{E. Running Simulations and Data Analysis}

During the acoustic Field II simulation, 256 lines were used to cover the entire field of view (approximately $36 \mathrm{~mm}$ ). A high-performance computing (HPC) cluster was used 
(http://superior.research.mtu.edu/). One beam line took approximately 10 minutes under MATLAB (Version 2013b, Mathworks Inc. MA, USA; 4 CPUs [Intel Xeon E2560; $2.8 \mathrm{GHz}]$ and $16 \mathrm{~GB}$ of memory). All other data preparation, analyses, and visualization were done by a high-end workstation (Dell Precision R5600, Dell Inc., Austin, TX with 64 GB memory).

\section{E.1. Speckle Tracking and Strain Estimation}

Once a pair of pre- and post-deformation radio-frequency (RF) echo frames were obtained (Section C), a modified block matching algorithm (MBMA) [75] was used to obtain axial and lateral displacements within a region of interest (ROI). The tracking kernel size is approximately $1.5 \mathrm{~mm}$ (lateral) $\times 0.6 \mathrm{~mm}$ (axial). More details of this method can be found in a previous publication [75]. In this study, we estimated local strains using a leastsquare method [76] using a linear regression window of $1.6 \mathrm{~mm}$.

\section{E.2. Estimation of Motion Tracking Accuracy}

Normalized cross correlation (NCC) between a pair of pre-deformation and motioncompensated post-deformation RF echo fields [77] was chosen to estimate motion tracking accuracy. Motion compensation here is referring to a process in which ultrasonically estimated displacements between the pre- and post-deformation ultrasound echo data were applied onto the post-deformation ultrasound data so that the post-deformation ultrasound data were warped back onto the coordinates of the pre-deformation echo data. In other words, the process of motion compensation was used to register two RF echo fields with apparent deformation. A higher NCC value between the two registered echo fields implies that the apparent deformation has been more accurately tracked. 


\section{E.3. Estimation of Retardation Time}

From time series of RF echo frames simulated during the simulation of viscoelastic strain imaging, a series of $\mathrm{K}$ strain images were first estimated as described above. Then, a time-resolved strain curve for each and every pixel was obtained as shown in Fig. 2.4. Each time-resolved strain curve was fitted into a first-order discrete Kelvin-Voigt (K-V) rheological model [78] using the Optimization Toolbox in MATLAB (Mathworks Inc., MA, USA). The formula of strain creep under the K-V model was below:

$$
\varepsilon(t)=\varepsilon_{0}+\varepsilon_{1}\left[1-\exp \left(-t / T_{1}\right)\right]
$$

where $\varepsilon_{0}$ is the instantaneous elastic strain that occurs immediately after compression, $\varepsilon_{1}$ is the amplitude of the exponential creep curve. In Eqn. (1), the constant $\mathrm{T}_{1}$ is the retardation time of the curve characterizing the delay in the full strain response, as illustrated in Fig. 2.4.

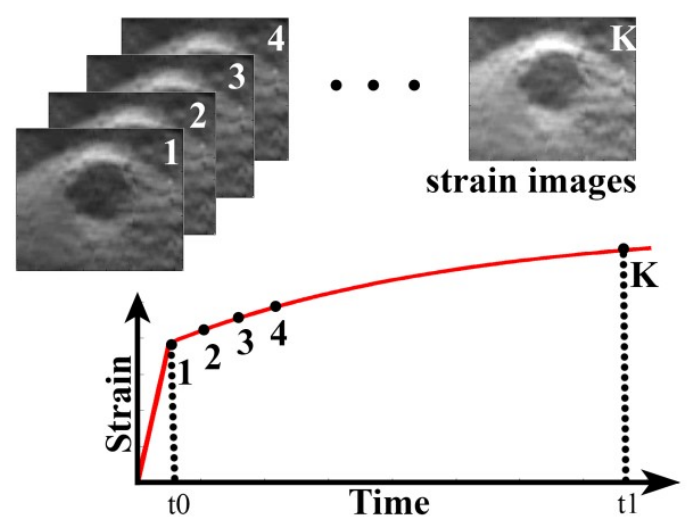

Figure 2.4. A schematic illustration of viscoelastic parameter estimation. A compressive force was applied within 1 second (i.e. t0 $=1$ second) at the beginning. Then, the compression was held until $\mathrm{t} 1$ (i.e. $\mathrm{t} 1=15$ seconds). Given $\mathrm{K}$ estimated strain images for the entire process. In this study, $\mathrm{K}$ is 15 , denoting 1 second per strain image. The red curve in the bottom plot is a representative time-resolved strain curve. The retardation 
time of the time-resolved strain curve was used to estimate the retardation time based on the K-V strain creep model.

\subsection{Simulated B-mode Images Based On Complex Patterns In Heterogeneous Media}

Three volumes of interest were selected from the un-deformed breast phantom (Fig. 2.1a) to perform acoustic simulations. Those three volumes were intended to show: 1) inclusion of a hypoechoic breast lesion 2) a complex "grape-like" structure of milk-ducts and 3) a complex layered structure of Cooper's ligaments embedded in the subcutaneous fat layer. All corresponding reflection coefficient and B-mode images are shown in Figs 2.5-2.7. Only center slices of the simulated reflection coefficient fields are shown.

In Figs. 2.5a-b, a hypoechoic lesion was surrounded by a thin (approximately 0.5 mm thick) hyper-echoic layer that is a simulated layer of lesion encapsulation. In Figs. 2.6a-b, a dark "grape-like" structure was clearly visible and embedded within the simulated fibro-glandular tissue region. In Figs. 2.7a-b, a complex layered structure mimicking Cooper's ligaments can be seen.
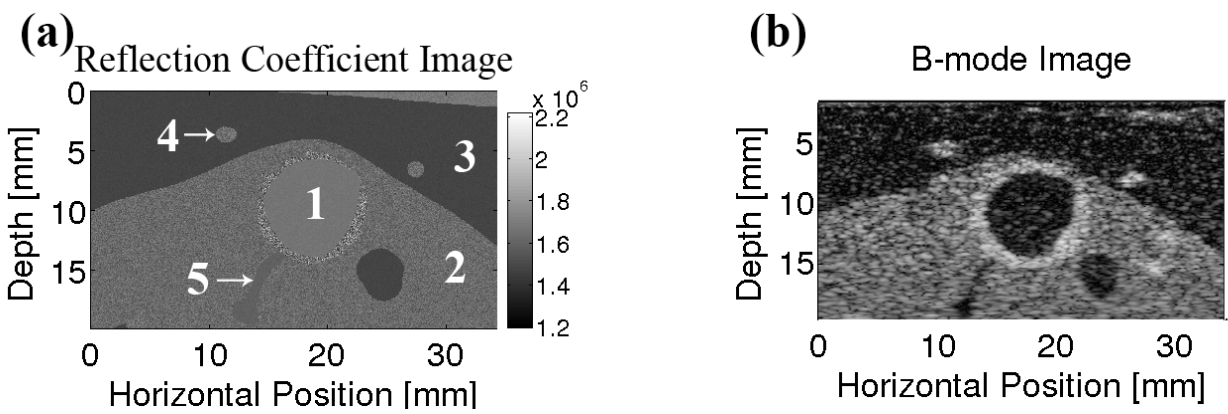

Figure 2.5. A simulated hypoechoic breast lesion: (a) acoustic reflection coefficient image and (b) a simulated B-mode image by Field II. In (a), 1-5 denote lesion, fibro-glandular tissue, breast fat, Cooper's ligaments and milk ducts, respectively. 

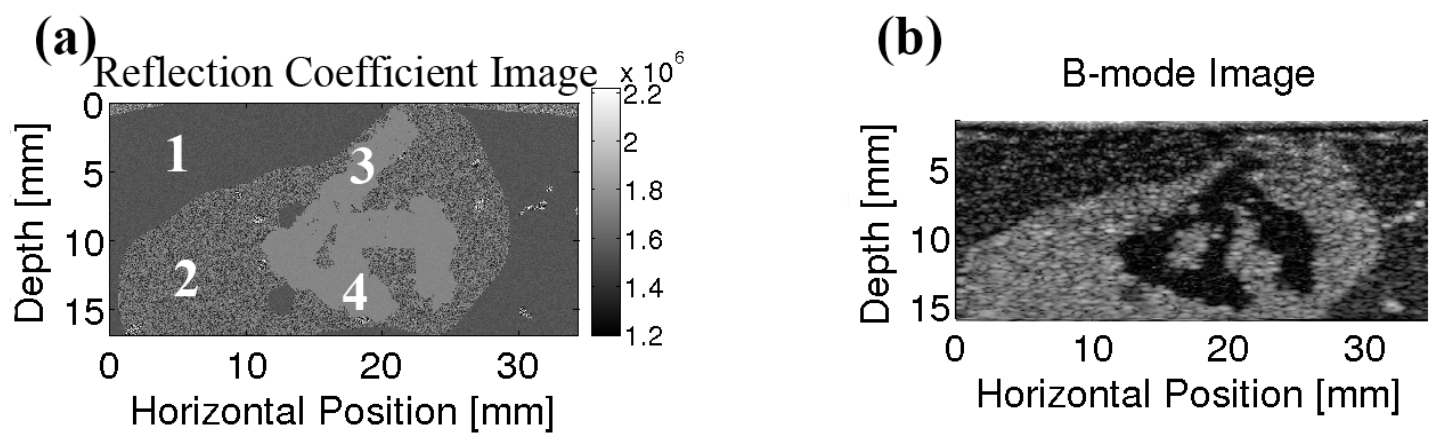

Figure 2.6. A simulated milk-duct structure embedded into the fibro-glandular layer: (a) acoustic reflection coefficient image and (b) a simulated B-mode image by Field II. In (a), 1-4 denote breast fat, fibro-glandular tissue, milk ducts, and lobe, respectively.

(a)

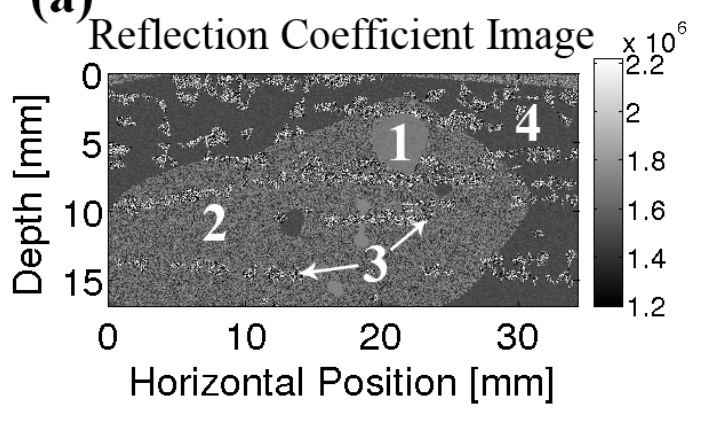

(b)

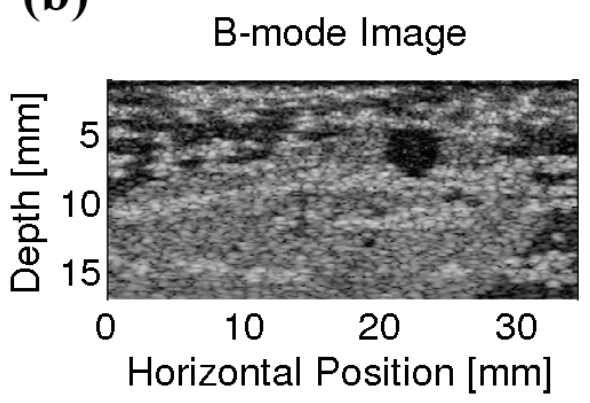

Figure 2.7. A simulated Cooper's ligament structure: (a) acoustic reflection coefficient image and (b) a simulated B-mode image by Field II. In (a), 1-4 denote lesion, fibroglandular tissue, breast fat, cooper's ligaments and breast fat, respectively.

\subsection{QUE Applications}

The first example was to show breast lesions with complex shapes and compositions can be readily integrated into the proposed virtual simulation platform. In the second example, a recent advance in nonlinear QUE was simulated using strain imaging, while the viscoelasticity assessment of breast tissues was demonstrated in the third example below. 


\section{Example 1: Simulations of Complex Breast Lesions}

Four breast phantoms were created; each contained a simulated breast lesion as shown in Figs. 2.8a, 2.9a, 2.10a and 2.11a, respectively. Hereafter, we will refer them as to Lesion 1-4 phantoms, respectively. The first two breast lesions had relatively simple (spherical) shapes and were designed to simulate benign breast tumors. Particularly, the first breast lesion (Fig. 2.8a) was a simulated solid tumor, whereas the second breast lesion (Fig. 2.9a) was originated from a milk duct, resulting in a solid lesion where milk ducts were embedded (see the red colored ducts in Fig. 2.9a). This simulated lesion might be similar to an early stage ductal carcinoma in situ (DCIS). The last two breast lesions had more complex shape and were designed to mimic breast cancers. The third one was "star"shaped and contained several protrusions mimicking cancer invasion. In the last one, a simulated necrotic region (see the red colored zone in Fig. 2.11a) was generated with a simulated breast lesion with a complex shape.

Simulation results of those 4 breast phantoms are shown in Figs. 2.8-2.11. All results were obtained using a $2 \%$ compression from the undeformed geometry. The corresponding acoustic reflective coefficients are displayed in Fig. 2.8b-2.11b, respectively for those 4 phantoms. Of note, Figs. $\mathbf{2 . 8 b}$-2.11b illustrate the center slice of those 4 reflective coefficient fields, respectively. The resultant B-mode images are shown in Fig. 2.8c-2.11c, respectively. The FE-simulated axial displacements induced by the 2\% compression are shown in Figs. 2.8d-2.11d, respectively. The ultrasonically-estimated axial displacements are shown in Figs. 2.8e-2.11e, respectively. All estimated axial 
(parallel to the acoustic beam direction) strain images can be seen in Figs. 2.8f-2.11f, respectively.
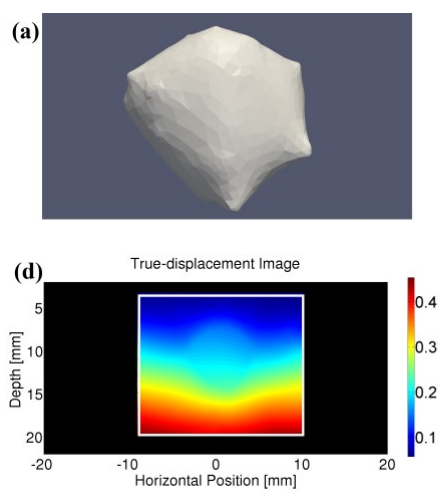
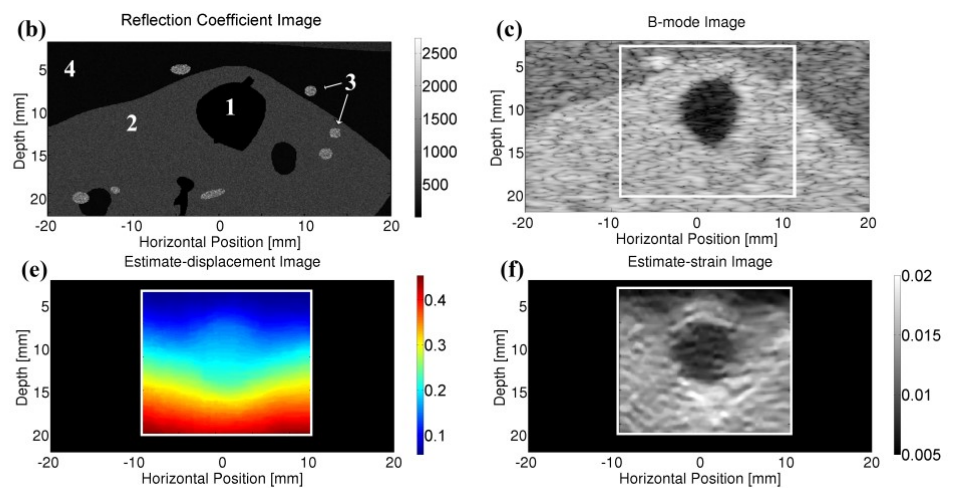

Figure 2.8. Results from the Lesion 1 phantom: (a) geometry, (b) reflection coefficient image, (c) B-mode image simulated by Field II where the white rectangle depicts a region of interest (ROI), (d) FE-simulated axial displacement in ROI, (e) ultrasonically estimated axial displacement image and (f) estimated axial strain image based on (e). The displacement color bars are in millimeters. In (b), 1-4 denote lesion, fibro-glandular tissue, cooper's ligaments and breast fat, respectively.
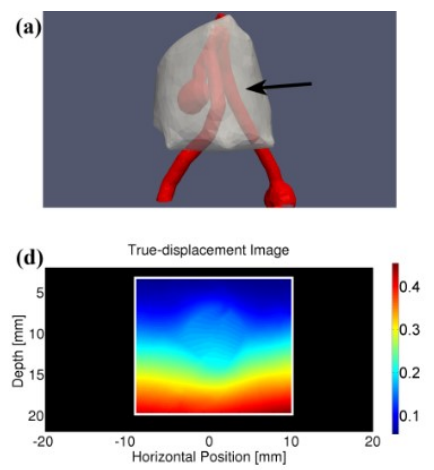
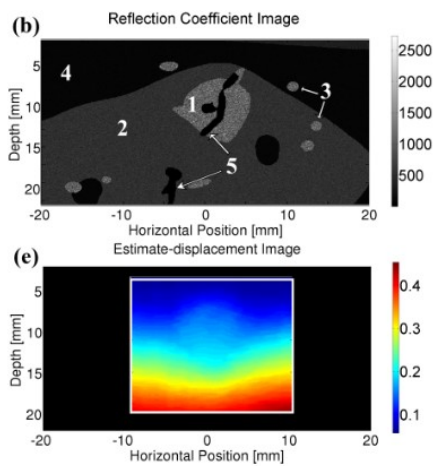
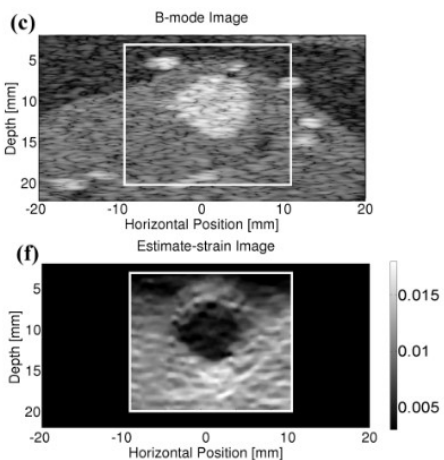

Figure 2.9. Results from the Lesion 2 phantom: (a) geometry, (b) reflection coefficient image, (c) B-mode image simulated by Field II where the white rectangle depict a region of interest (ROI), (d) FE-simulated axial displacement in ROI, (e) ultrasonically estimated axial displacement image and (f) estimated axial strain image based on (e). The displacement color bars are in millimeters. In (a), the red colored object is a cluster of milk ducts (see arrow). In (b), 1-4 denote lesion, fibro-glandular tissue, cooper's ligaments and breast fat, respectively. 

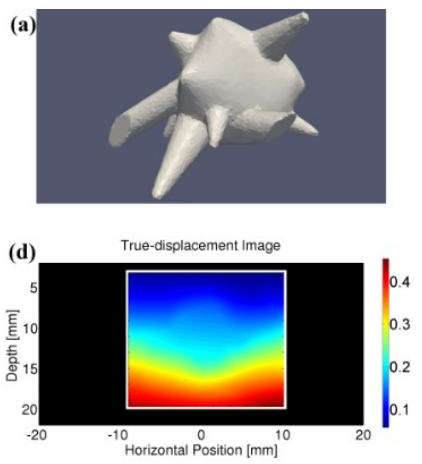
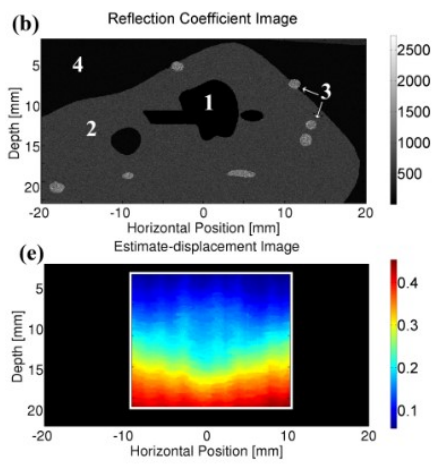
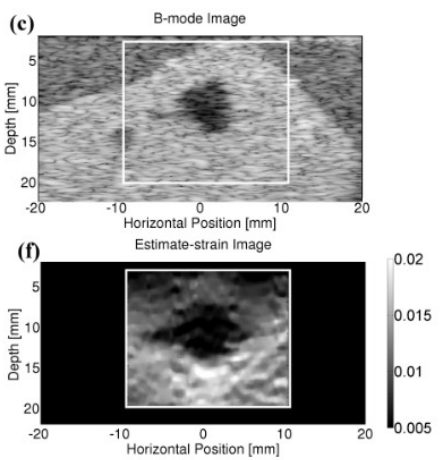

Figure 2.10. Results from the Lesion 3 phantom: (a) geometry, (b) reflection coefficient image, (c) B-mode image simulated by Field II where the white rectangle depict a region of interest (ROI), (d) FE-simulated axial displacement in ROI, (e) ultrasonically estimated axial displacement image and (f) estimated axial strain image based on (e). The displacement color bars are in millimeters. In (b), 1-4 denote lesion, fibro-glandular tissue, cooper's ligaments and breast fat, respectively.
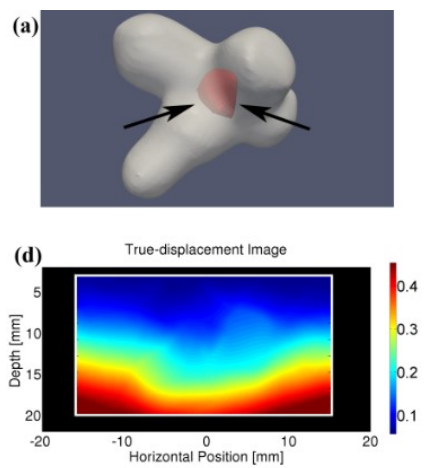
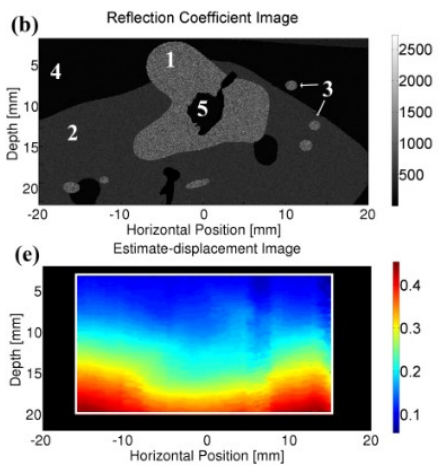
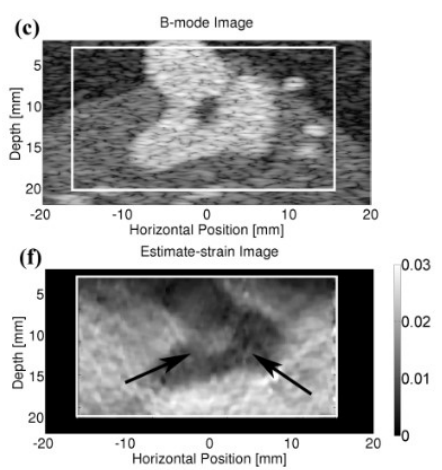

Figure 2.11. Results from the Lesion 1 phantom: (a) geometry, (b) reflection coefficient image, (c) B-mode image simulated by Field II where the white rectangle depict a region of interest (ROI), (d) FE-simulated axial displacement in ROI, (e) ultrasonically estimated axial displacement image and (f) estimated axial strain image based on (e). The displacement color bars are in millimeters. In (a), the red-color region is a simulated necrotic zone inside the simulated cancer. In (b), 1-4 denote lesion, fibro-glandular tissue, cooper's ligaments and breast fat, respectively. In (a) and (f), arrows point to the simulated necrotic zone.

All 4 simulated breast lesions were clearly visible in respective B-mode and strain images. Their overall sizes and shapes were visually consistent with all phantom designs. 
Particularly, the (soft) necrotic region in the Lesion 4 phantom was also seen on the corresponding strain and B-mode images. However, the small cluster of (soft) milk ducts was not shown in both the strain and B-mode images.

Estimate NCC values from a range of deformation $(0 \%-6 \%)$ for Lesion 1 phantom were compared with clinically scanned in vivo data and data from a simple tissuemimicking phantom, as reported by Jiang et al. [77] As shown in Fig. $\mathbf{2 . 1 2}$ below, the calculated NCC values from simulated Lesion 1 phantom were lower than NCC values obtained from the tissue-mimicking phantom but were higher than NCC values obtained from clinically-scanned in vivo data [77]. Results from other three phantoms were comparable.

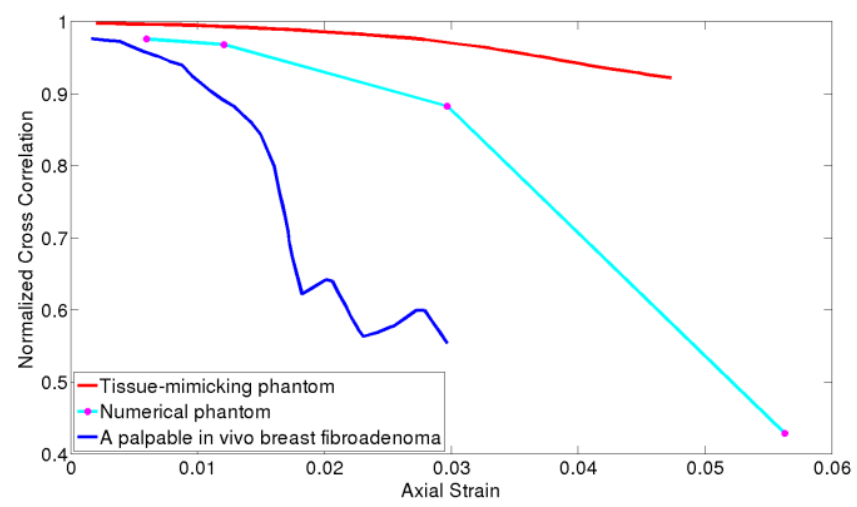

Figure 2.12. A plot of estimated NCC values between the pre- and post-deformation RF echo fields under a range of deformation ( $0-5 \%$ compression). Data of the tissuemimicking phantom and the in vivo breast fibroadenoma were replicated from a previous publication [77].

\section{Example 2: Nonlinear QUE via Strain Imaging}




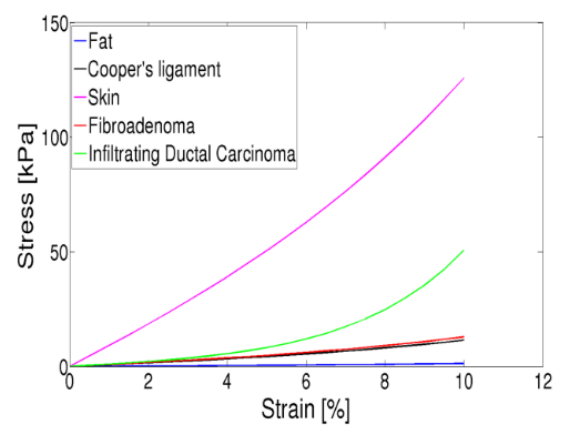

(a) Stress-Strain Curves

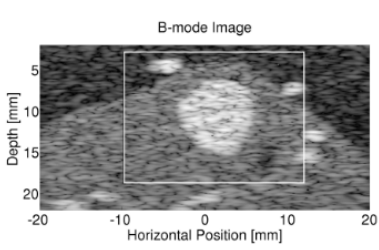

Incremental strain contrast:

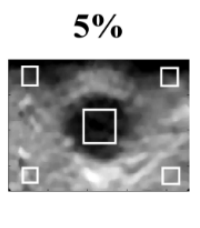

1.82

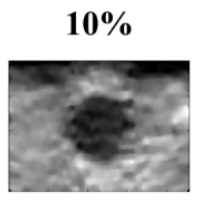

1.50

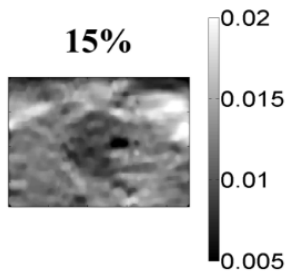

1.29

(b) Fibroadenoma
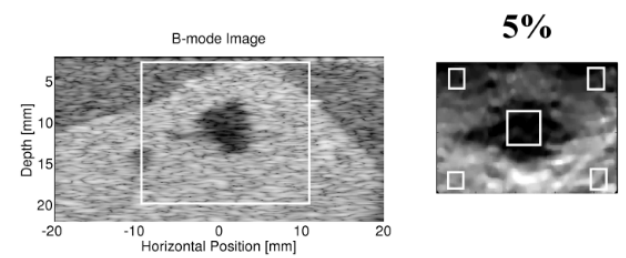

1.96

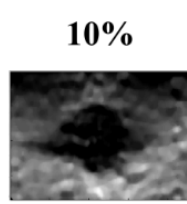

1.94

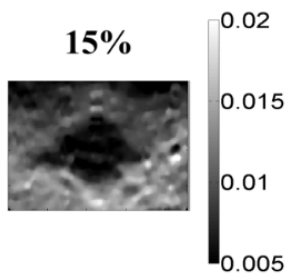

1.75

(c) Infiltrating Ductal Carcinoma

Figure 2.13. (a) Stress-strain data of the components in breast tissue. In (b) and (c) are Bmode (left) and three incremental strain images for lesions. The regions of interest (ROI) for strain contrast analysis are shown in the strain image in (b) and (c). The lesion ROI is in the middle, the background ROIs are placed near the corner to diminish the influence of the stress concentration. The area of lesion ROI is equal to the area of background ROIs. The incremental strain contrast is the ratio of strain in background ROIs and lesion ROI.

QUE simulations were performed for Lesion 1 and Lesion 3 phantoms up to $20 \%$ of deformation. Stress-strain curves used for the FE-simulations are shown in Fig. 2.13a. Detailed hyperelastic parameters associated with those curves can be found in Appendix $\underline{\text { B }}$ (see Table B1). B-mode and incremental ( $2 \%$ frame-averaged) axial strain images at 
varying preload of the Lesion 1 and Lesion 3 phantoms are shown in Figs. 2.13b and 2.13c, respectively. The values appearing above each strain image indicate the accumulated (preloading) axial strains. In the Lesion 1 phantom, at low preload, the simulated breast tumor is stiffer (darker) than the background glandular tissue. At modest ( 10\%) preload, the simulated tumor loses visibility with the increase of the pre-load level. In the Lesion 3 phantom, the visibility of the simulated cancer in strain images remains stable as the increase of pre-loading. Strain contrast values were defined as the ratio between averaged background strain and averaged inclusion strain from equal areas. Calculated contrast values were also shown in Figs. 2.13b and 2.13c, and were consistent with the visual perception.

\section{Example 3: Time-dependent QUE via Strain Imaging}

In this example, the Lesion 1 phantom (Fig 2.8a) was simulated using two different pre-determined values for retardation time. The retardation time for these two cases was estimated using the method described before (Section E in the Methods). From Figs. 2.14a and $\mathbf{2 . 1 4 b}$, the distinction between the assessed retardation-time is clearly visible.
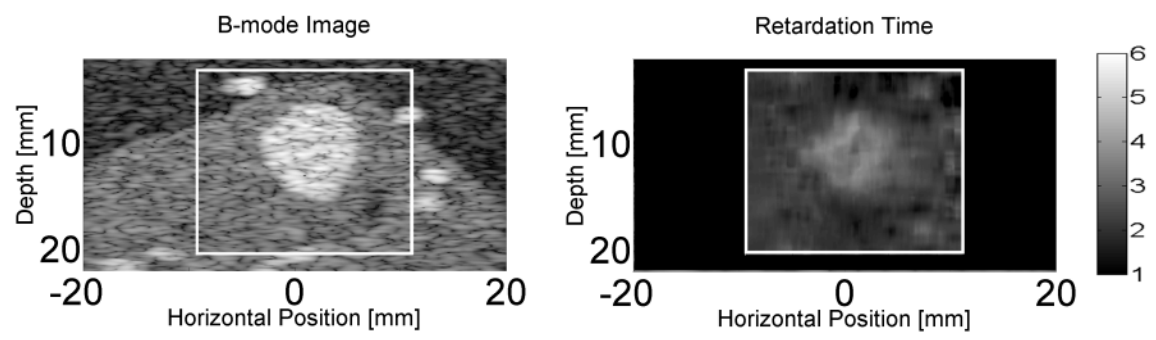

Figure 2.14 Images showing the assessed retardation time for the Lesion 1 phantom: (a) B-mode image and (b) the estimated $\mathrm{T}_{1}$ image. In (b), the pre-determined $\mathrm{T}_{1}=4$ seconds for the Lesion 1 while the averaged $\mathrm{T}_{1}$ from the Lesion 1 was 3.5 second. The unit for the color bar in (b) is second. 


\subsection{Discussion}

Like many new diagnostic imaging systems, tissue-mimicking phantom development for QUE has aided in testing prototype QUE systems. However, we found that many QUE algorithms perform well and show great promise in phantom experiments but fail to live up to expectations during clinical trials. This is largely because the underlying motion in tissue-mimicking phantom experiments is too simple and uniform whereas that found in in vivo tissue motion during clinical trials is significantly more challenging. An NCC value between a pair of pre-deformation and motion-compensated post-deformation RF echo fields [77] has been used to assess motion tracking accuracy. Estimated NCC values may also be used as a metric indicating the challenging level of motion tracking. Consequently, numerical breast phantoms presented in this paper resulted in echo data that have challenged our motion tracking algorithm [75] more as compared to a physical tissue-mimicking phantom. However, this is only our first attempt. More features such as sliding and shearing [79] between tissue interfaces and acoustic attenuation can be further incorporated into the proposed simulation platform to challenge motion tracking algorithms. It is our goal to further develop the virtual platform so that the proposed platform can be used to perform virtual QUE clinical trials in a cost-effective manner. With the availability of those simulated known but complex QUE data, this platform could also be used as a training tool for clinicians to improve their confidence on image interpretation. The platform is still evolving and adding simulation capabilities for shear wave elastography [16] is our priority, though this development is far from being trivial. For instance, in order to reliably simulate the shear wave propagation in 
heterogeneous media, additional wave-absorption conditions and numerical stabilization schemes may need to be implemented to FEBio [61]. Those features are not currently available in FEBio [61]. We intend to make all relevant computer code and ultrasound data freely available to the research community upon request. In the next stages, our goal is to make all source code freely available through Github. Thanks to the open science nature of this simulation platform, the sophistication of the proposed virtual breast phantom can be considerably enhanced once more anatomical features are incorporated and more research groups become involved with this work.

The small cluster of milk ducts could not be seen in both B-mode and strain images, largely due to its small size (approximately $2-\mathrm{mm}$ diameter). The beam profile of the simulated transducer suggested that the axial and lateral beam widths were $0.8 \mathrm{~mm}$ and 2.1 $\mathrm{mm}$ at full width and half maximum (FWHM), respectively. It is also interesting to note that the estimated values of retardation time slightly deviated from the pre-determined values, though the image contrast was obvious (see Fig. 2.15). This is largely since our method used to estimate the $T_{1}$ is not optimized.

\subsection{Conclusion}

In summary, a virtual ultrasound simulation platform mainly based on open-source software has been initially implemented and tested in this study. Our preliminary results suggest that this virtual ultrasound simulation platform can be used to perform both acoustic and QUE simulations. One significant contribution of this work is the fact that, to our knowledge, advanced QEU simulations of this kind (i.e. realistic geometries and, tissue composition and properties) have not been reported elsewhere. Furthermore, two advanced 
QEU applications, namely nonlinear [71, 72] and time-dependent [78] strain imaging, were used as showcase examples to demonstrate capabilities of the proposed system. Thus, another intellectual contribution is that the proposed software innovation enables imaging physicists/scientists to rigorously compare image pixels with underlying soft tissue properties (also known as "ground truth") using simulated ultrasound data emulating in vivo breast anatomy. Such comparisons are highly desirable and critically important for new applications such as nonlinear [71, 72] and time-dependent [78] strain imaging. 


\section{Chapter 3. Virtual Simulation Platform for Acoustic Radiation Force-based Breast Elastography ${ }^{2}$}

\subsection{Workflow of Virtual Simulation Platform for Acoustic Radiation Force-based UE}

As shown in Fig. 3.1, the proposed software platform for acoustic radiation forcebased UE involves several key (open-source or free) software packages in multiple steps. FIELD II [80] is an ultrasound simulator that was used to (1) calculate distributions of acoustic pressure and (2) generate ultrasound echo signals given a weakly scattering medium. Using the acoustic pressures as boundary conditions, FEBio [81], an open-source finite element solver for biomechanics, was employed to obtain local tissue deformation. Once a series of pre- and post-deformation ultrasound echo data were obtained, speckle tracking [82] was performed to estimate tissue deformation and subsequently assess tissue elasticity, dependent on the modality being simulated. Image formation procedures in pSWE, ARFI and SSI are well documented in the literature [2].

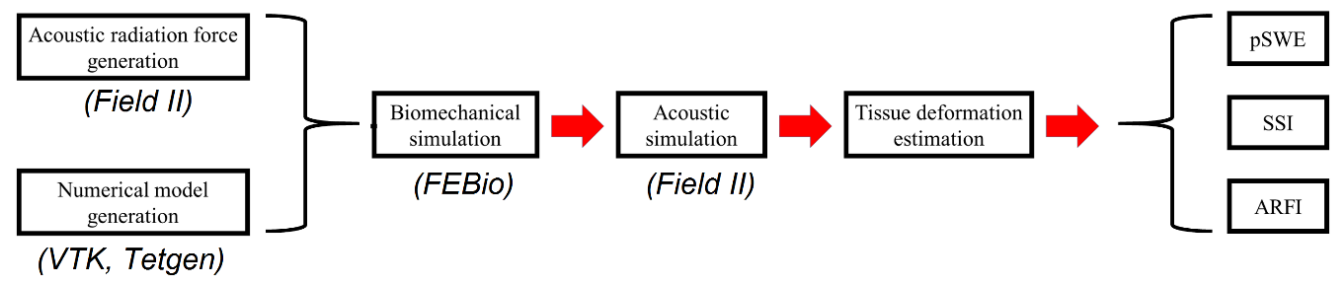

Figure 3.1. A graphic illustration of the proposed virtual simulation platform for acoustic radiation force-based UE.

\section{A. Calculation of Acoustic Radiation Force}

\footnotetext{
2 The material contained in this chapter was previously published in Physics in Medicine and Biology, 2017 Feb;62(5):1949-1969. Refer Appendix G for granted permission to be republished.
} 
Using a high-frequency $(5-10 \mathrm{MHz})$ linear array transducer, distributions of acoustic pressure were simulated using FIELD II [80]. An example of FIELD II simulated acoustic pressure field obtained using a $10 \mathrm{MHz}$ linear array transducer was shown in Fig. 3.2. Ultrasound parameters of a $5 \mathrm{MHz}$ and a $10 \mathrm{MHz}$ linear array probes were the same as ones used by Palmeri and Nightingale [16] and Zhao et al. (Zhao et al., 2011), respectively, Those ultrasound simulation parameters are listed in Table 3.1. For subsequent FEA simulations to obtain local tissue deformation, the only acoustic pressure within the beam width (full width at half maximum) was used to deform subsequent finite element models, as suggested by Palmeri et al. [44]. Normalized acoustic pressures were first projected onto FEA Models and then their amplitudes were scaled to achieve peak displacements of 15 micrometers [44] in the proximity of the simulated acoustic pushing pulse.

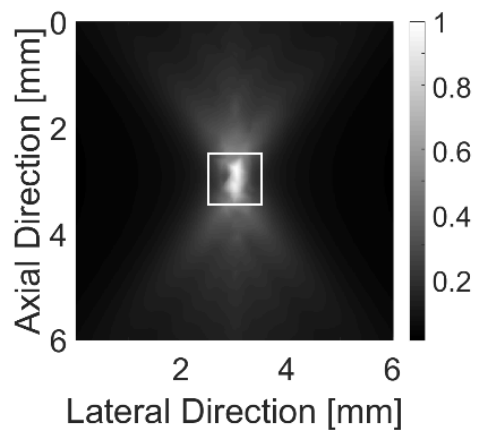

Figure 3.2. A plot of an acoustic intensity field produced by FIELD II. Only the center slice is displayed. The focal region (see the white rectangle; approximately $1 \mathrm{~mm} \times 1$ $\mathrm{mm} \times 1 \mathrm{~mm}$ cube in 3D) is projected onto the numerical breast models to excite SW propagation or displace adjacent tissues. The color bar represents normalized values from 0 to 1 . 
Table 3.1. A list of system parameters of a (simulated) linear array ultrasound transducer used for pushing and tracking beams. These parameters were adopted from two published papers $[18,50]$. Both linear array transducers were used for FIELD II simulations.

\begin{tabular}{ccc}
\hline System Parameters & Values based on & Values based on \\
\hline \hline Excitation Frequency (MHz) & {$[18]$} & {$[50]$} \\
Tracking Frequency (MHz) & 5 & 10 \\
Sampling Frequency (MHz) & 5 & 10 \\
Bandwidth (\%) & 50 & 50 \\
Element Width (mm) & 50 & 50 \\
Element Height (mm) & 0.2 & 0.1 \\
Spacing Between Elements (mm) & 5 & 5 \\
Number of Active Elements & 0 & 64 \\
Tracking pulse repetition frequency & $32,64,96$ & 10 \\
(kHz) & 4 & 0.1 \\
\hline
\end{tabular}

\section{B. Numerical Phantom Generation}

Three different numerical breast models of increasing complexity (i.e. a simple uniform medium [Model 1], a uniform medium with an (8-mm diameter) spherical inclusion [Model 2] and a complex breast model derived from magnetic resonance imaging data [Model 3]) were used in this study, as shown in Fig. 3.3. Model 1 and Model 2 were built to replicate tissue-mimicking phantoms reported in the literature $[18,44,83]$. Details of the complex breast model (i.e. Model 3) can be found in our previous publication [84]. To improve numerical accuracy for simulations of SW propagation, all numerical models 
in this study were meshed by Tetgen [59] with quadratic tetrahedrons (i.e. 10 node tetrahedrons). Of note, the selection of quadratic tetrahedral (finite) elements comes with higher computing expenses but offers improved accuracy as compared to linear tetrahedrons (i.e. 4 node tetrahedrons). Mesh sensitivity was tested using two computing grids: 1.1 million and 0.5 million (quadratic) tetrahedrons. The displacement differences are less $10^{-3}$ micrometers between the coarse and fine meshes. Therefore, the coarse mesh was used for subsequent biomedical simulation.

In the coarse mesh, adaptive meshing [59] was used and the average element size near the inclusion and the pushing beam was on the order of $0.01 \mathrm{~mm}^{3}$. Such a fine (mesh) density allowed accurate simulations of mechanical responses induced by the acoustic radiation forces.

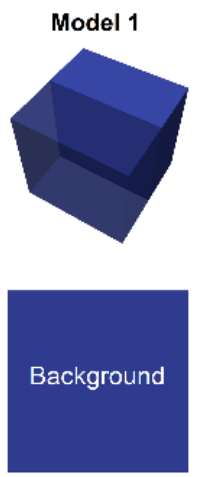

(a)

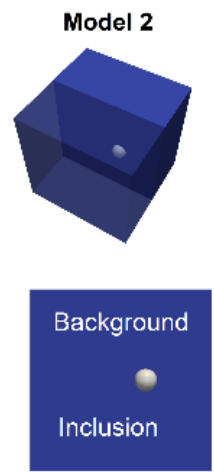

(b)
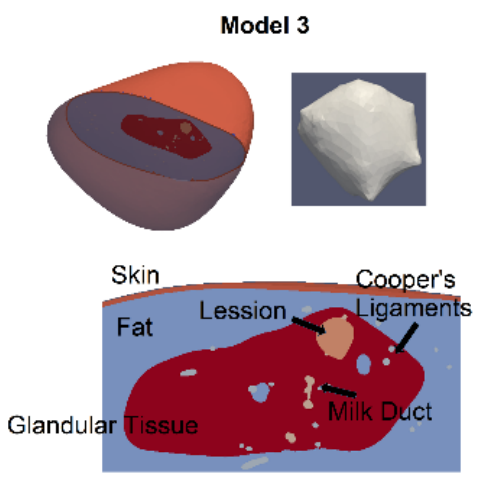

(c)

Figure 3.3. Three numerical breast models of increasing complexity: (a) a uniform medium ( $40 \mathrm{~mm} \times 40 \mathrm{~mm} \times 40 \mathrm{~mm}$, approximately 0.5 million finite elements), (b) a uniform medium $(60 \mathrm{~mm} \times 60 \mathrm{~mm} \times 60 \mathrm{~mm})$ with a spherical inclusion $(8 \mathrm{~mm}$ diameter; approximately 0.5 million finite elements), and (c) a complex breast model $(62 \mathrm{~mm} \times$ $68 \mathrm{~mm} \times 41 \mathrm{~mm}$ ). In Model 3, the irregular lesion has a diameter of approximately 5 $\mathrm{mm}$ and the final mesh size is 2.0 million quadratic tetrahedrons. The first and second rows are $3 \mathrm{D}$ geometries and center slices, respectively. 


\section{Biomechanical Simulation}

To simulate tissue deformation induced by the acoustic radiation force/pressure (see Fig. 3.2). FEBio [81], an open source FEA package, was used to perform biomechanical simulations. Given the small deformation (on the order of 1-15 micrometers) induced by the acoustic radiation forces [2, 85], all materials used were assumed to be isotropic and linearly elastic as a first approximation. In FEBio, the NeoHookean model [86] closely approximates linearly elastic media under the small deformation. Material properties of Models 1-3 are listed in Table 3.2. Particularly, material properties of Models 1 and 2 were selected to match published tissue-mimicking phantom experiments $[18,44,83]$ for subsequent comparisons, while material properties of Model 3 were chosen to reflect the fact that the elastic contrast between a breast lesion and its background is approximately $8-11[87,88]$ at small strains (e.g. 1-5\%). The mass density of all materials used in this study was set to $1.0 \mathrm{~g} / \mathrm{cm}^{3}$. In all models, dimensions of those three models were large enough so that shear wave reflections from exterior edges were avoided since "non-reflective" boundary condition has not been implemented in FEBio. More detailed descriptions related to pSWE, SSI and ARFI are briefly summarized below.

Table 3.2. Mechanical properties of the three numerical breast models. Young's modulus values were extracted from three publications $[18,44,83]$ and all materials were assumed to be nearly incompressible.

\begin{tabular}{ccc}
\hline & Young's \\
modulus & Poisson's \\
$(\mathrm{kPa})$ & Ratio \\
\hline \hline
\end{tabular}




\begin{tabular}{cccc}
\hline \hline $\begin{array}{c}\text { Model 1 } \\
\text { [44] }\end{array}$ & Background & 4.76 & 0.499 \\
\hline $\begin{array}{c}\text { Model 1 } \\
\text { [44] }\end{array}$ & Background & 4.61 & 0.499 \\
\hline $\begin{array}{c}\text { Model 2 } \\
{[83]}\end{array}$ & Background & 15 & 0.499 \\
& Inclusion & 60 & 0.499 \\
\hline & Breast fat & 10 & 0.499 \\
Model 3 & Cooper's ligament & 75 & 0.499 \\
{$[87,88]$} & Milk duct & 10 & 0.499 \\
& Lesion & 150 & 0.499 \\
& Skin & 400 & 0.499 \\
& Fibro-glandular tissue & 15 & 0.499 \\
\hline
\end{tabular}

\section{(1) Point Shear Wave Elastography (pSWE)}

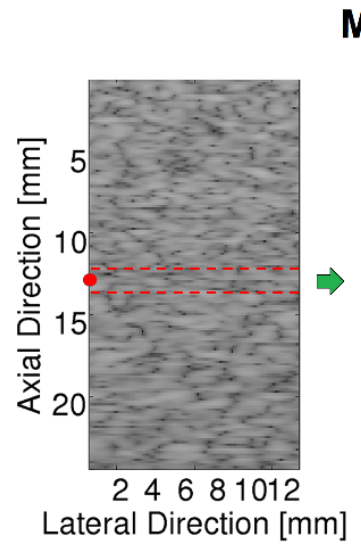

(a)

\section{Model 1}

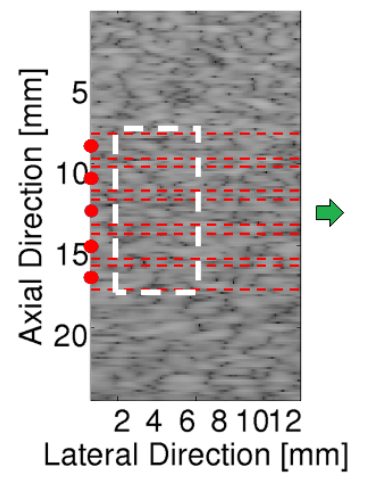

(b)

Figure 3.4. A graphic illustration of the simulation setting for virtual pSWE in Model 1. B-mode image simulated using FIELD II is displayed in (a) and (b). In (a), simulated acoustic radiation forces were projected onto the red dot region. The green arrow is the SW propagation direction. The horizontal red dashed lines represent the depth $(1 \mathrm{~mm})$ over which shear wave reconstructions were performed for pSWE. Repeating (a) along the axial depth direction, a two-dimensional (2D) SWS image within the white rectangle can be obtained for a large region of interest (ROI) as shown in (b). 
Acoustic radiation force fields were projected onto Model 1 through cubic spline interpolations in MATLAB (Mathworks Inc., MA, USA), as shown in Fig. 3.4 (red dot region). The acoustic pulse was used to excite the tissue (see the red dots in Fig. 3.4a-b) for 1 millisecond [44] while the entire (temporal) length of the dynamic FEA simulation was 8 milliseconds. The normalized acoustic pulse (see Fig. 3.1) was scaled so that the peak displacement at the focal point was around $15 \mu m$ [16]. The time-resolved displacement field between two horizontal red dashed lines (a $1 \mathrm{~mm}$ spacing) in Fig. 3.4a was stored and subsequently analyzed to estimate SWS values. To obtain a SWS map in two-dimension (2D), this process was repeated for different depths (every $1 \mathrm{~mm}$; see Fig. 3.4b).

\section{(2) Supersonic Shear Imaging (SSI)}

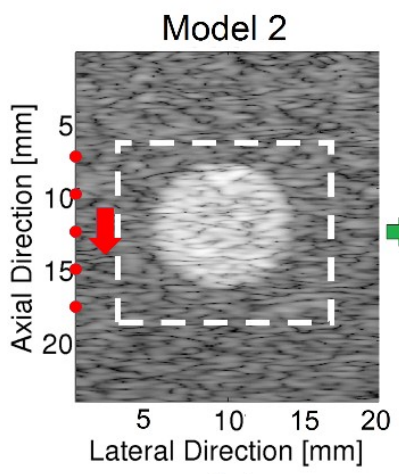

(a)

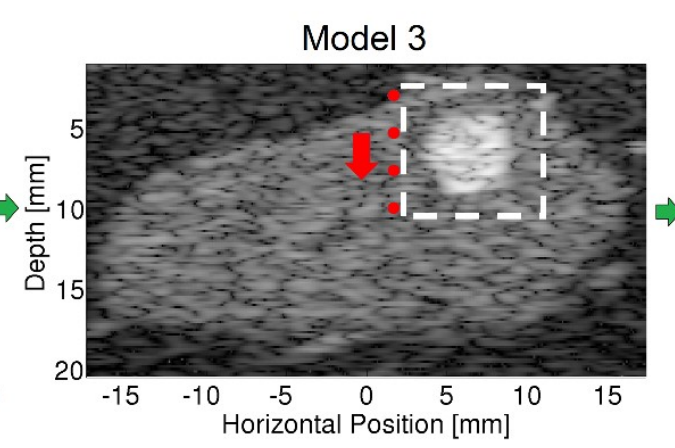

(b)

Figure 3.5. A graphic illustration of the virtual SSI setting for two models: (a) Model 2 and (b) Model 3. Two FIELD II simulated B-mode images were displayed to illustrate compositions of Models 2 and 3, respectively. To generate supersonic sources, acoustic radiation forces were projected onto a series of excitation locations with a predetermined time delay in a sequence. Red dots in each plot indicate those supersonic sources used to generate the SSI image. The red arrows denote the sweeping (i.e. delayed excitation in a sequence) direction of acoustic radiation forces, while green arrows indicate the SW propagation direction. The white rectangle is the region of interest (ROI). 
To approximate plane SW propagation in SSI, impulse acoustic radiation forces whose pulse length was 100 microseconds swept down along the axial depth direction (spaced $1 \mathrm{~mm}$ apart) successively [85]. The time delay between the above-mentioned sequential acoustic pushing was 10 microseconds to ensure the sweeping speed moved faster than the SW propagation speed [85]. Then, a set of time-resolved displacement fields in the region of interest (ROI) was acquired. FIELD II ultrasound simulations were performed to simulate ultrasound data acquisition with a frame rate of $10 \mathrm{KHz}$. The sweeping depth ranges were $15 \mathrm{~mm}$ (15 locations) and $10 \mathrm{~mm}$ (10 locations) for Models 2 and 3, respectively.

\section{(3) Acoustic Radiation Force Impulse (ARFI) imaging}

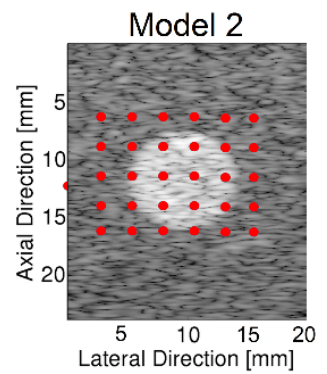

(a)

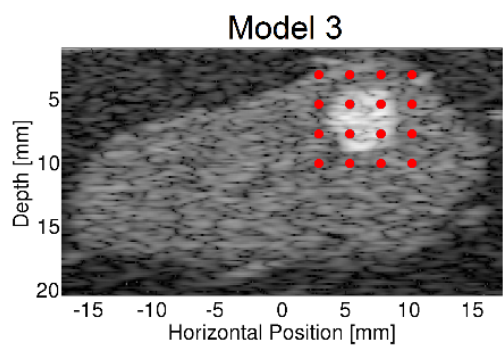

(b)

Figure 3.6. A graphic illustration of the setting of virtual ARFI imaging for two models overlaid with FIELD II simulated B-mode images: (a) Model 2 and (b) Model 3. Spatially localized acoustic pushes were applied onto respective ROIs sequentially (20 $\mathrm{mm} \times 15 \mathrm{~mm}$ and $10 \mathrm{~mm} \times 9 \mathrm{~mm}$ for Models 2 and 3, respectively). Each red dot indicates one pushing location used for the virtual ARFI imaging. The number of red dots used was solely for the illustration purpose.

Virtual ARFI simulations were performed in conjunction with Models 2 and 3. In virtual ARFI imaging, acoustic radiation forces with an acoustic pulse length of 100 microseconds were simulated to interrogate multiple spatial locations (spaced $1 \mathrm{~mm}$ apart 
in each direction) sequentially, as shown in Fig. 3.6. Each acoustic excitation was followed by 2 milliseconds of simulated tracking beams to observe the time-resolved tissue responses within the region of excitation [89]. This sequence was repeated over ROIs of $20 \mathrm{~mm} \times 15 \mathrm{~mm}$ and $10 \mathrm{~mm} \times 9 \mathrm{~mm}$ for Models 2 and 3, respectively. Time-resolved displacement fields were stored and were used in conjunction with FIELD II for the subsequent ultrasound simulations.

\section{Ultrasound Simulations Using FIELD II}

All ultrasound simulations were performed by FIELD II [80]. Procedures were the same as described in our previous paper [84] and were briefly summarized below. Randomly positioned point scatterers were placed in Models 1-3 to ensure Rayleigh scattering. The scatterer density was 30 scatterers $/ \mathrm{mm}^{3}$. Acoustic reflective coefficients were assigned to each point scatterer. The corresponding data were chosen based on the literature [90]. Perfect matching layers around the boundaries were automatically added by FIELD II to avoid undesirable wave reflections at the boundaries. Acoustic attenuation was not simulated. During FIELD II simulations, around 150 lines were used to cover the entire field of view, resulting in a beam spacing of $0.12 \mathrm{~mm}$ for all simulated ultrasound data.

\section{E. Data Analysis}

A high-performance computing cluster was used. One beam line took approximately 1 minute under MATLAB (Version 2013b, Mathworks Inc. MA, USA; 4 CPUs [Intel Xeon E2560; 2.8GHz] and 16GB of memory).

\section{(1) Speckle Tracking and Displacement Estimation}


Once a pair of pre- and post-deformation radio-frequency echo frames were obtained using the above-mentioned FIELD II simulation, a modified block matching algorithm [82] was used to obtain axial and lateral displacements within a region of interest (ROI). The tracking kernel size was approximately $1.5 \mathrm{~mm}$ (lateral) $\times 0.6 \mathrm{~mm}$ (axial). More details of this method can be found in a previous publication [82].

\section{(2) Shear Wave Speed (SWS) Estimation}

In this study, the time-of-flight (TOF) approach $[44,91]$ was used to estimate SWS in the region of interest (ROI). TOF-based methods employ a priori assumptions, including local homogeneity, and a known direction of propagation, such that the arrival time at adjacent positions can be used to determine the shear wave speed [16]. In this study, the middle point of the shear wave front was identified as a more reliable feature to estimate the shear wave arrival time at fixed lateral positions. The acoustic simulation data were upsampled to $50 \mathrm{kHz}$ (from $10 \mathrm{kHz}$ ) before being processed by the time-of-flight method [44]. To improve reconstruction results of the virtual SSI, a directional filter [83] was

employed to remove off-axis wave interferences among ultrasonically-tracked displacement data, prior to the application of the above-mentioned TOF method.

\section{(3) Evaluation of Elastic Contrast on Elastographic Images}

Elastic contrast (EC) [92] was chosen to compare results among different methods (i.e. pSWE, SSI, ARFI, and SE). The elastic contrast (EC) is defined as follows,

$$
E C=\frac{I_{t}}{I_{b}}
$$


where $I$ denotes the mean values of intensity on the elastogram of interest, and subscripts $b$ and $t$ represent the background and target, respectively. Two different methods for ROI selection were used as shown in Fig. 3.7a-b. In Fig. 3.7, the middle rectangular or circular ROI within the target (delineated by the contour in orange in Fig. 3.7a) was used to calculate the mean intensity of the target $\left(I_{t}\right)$, while small squares (Fig. 3.7a) or the large shaded blue ring (Fig. 3.7b) were used to calculate the mean intensity of the background $\left(I_{b}\right)$. The area of the target ROI is equal to its counterpart in the background. The "true" elastic contrast between the target and the background of the numerical phantom was calculated based on shear modulus values used in FEA simulations.

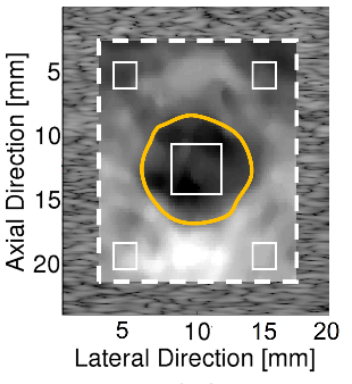

(a)

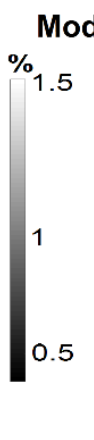

Model 2

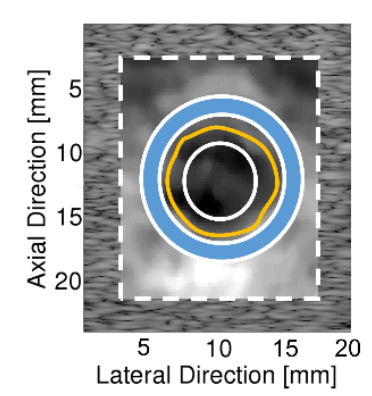

(b)

Figure 3.7. Illustrations of the process for the calculation of EC using an example strain elastogram. Two ROI selection methods (a)-(b) were used. The rectangles or circles depict respective ROIs in the background and the target. The target was delineated by the manually-segmented contour in orange color in both plots.

\section{(4) Study of Biases in pSWE}

In order to show the SWS measurements were dependent on the aperture size, focal depth and lateral tracking ranges, numerical simulations using a $5 \mathrm{MHz}$ linear array transducer were performed based on experimental data reported by Zhao et al. [18] All 
ultrasound simulation parameters can be found in Table 3.1 above. Particularly, the simulated pushing beam was focused on $20 \mathrm{~mm}$ and $40 \mathrm{~mm}$ depths, respectively; In each focal depth, three different aperture sizes (i.e. 32, 64 and 96 active elements) were simulated. All simulations were performed in a numerical phantom as described in Table 3.2 above.

How the lateral tracking range affects the SWS measurements was also investigated? When the simulated acoustic pushing beam was focused at the depth of 20 mm using three different active aperture sizes, the SWS values were estimated in two lateral tracking ranges: 1-6 mm (hereafter referred as to the near tracking range) and $8-13$ $\mathrm{mm}$ (hereafter referred as to the far tracking range) from the focus of the pushing beam.

\subsection{Virtual pSWE}

Results from the proposed virtual pSWE obtained from Model 1 were shown in Figs. 3.8 and 3.9, respectively. In Fig. 3.8, displacement data along different (temporal) phases have been displayed to visualize the SW propagation (see Figures 8b-d). In Fig. 3.8e, the estimated SWS along a lateral (perpendicular to the SW propagation direction) line was $1.31 \pm 0.04 \mathrm{~m} / \mathrm{s}$. The relative difference between the known SWS $(1.26 \mathrm{~m} / \mathrm{s})$ and the estimated SWS $(1.31 \mathrm{~m} / \mathrm{s})$ was approximately $4.0 \%$. The known SWS was estimated by $\sqrt{G / \rho}$ based on its predetermined material properties (see Table 3.2), where $G$ and $\rho$ are shear modulus and the mass density, respectively. 


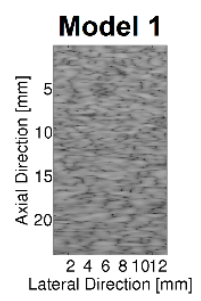

(a)

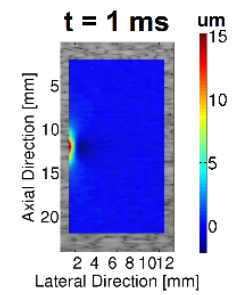

(b)

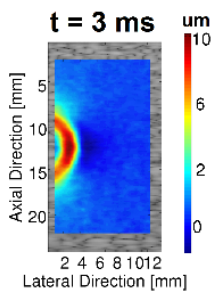

(c)

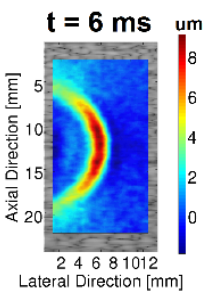

(d)

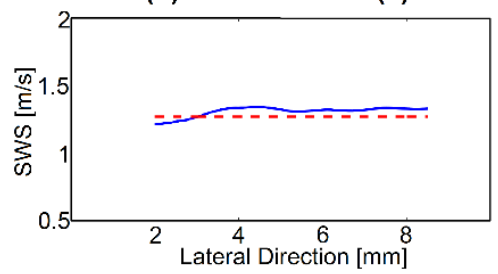

(e)

Figure 3.8. The SWS along lateral direction estimated from Model 1 by using pSWE: (a) a simulated B-model image, simulated displacement images at (b) 1 millisecond, (c) 3 milliseconds and (d) 6 milliseconds after the pushing pulse started, and (e) a plot of estimated SWS. In (e), the blue solid curve represents the estimated SWS, while the red dashed line represents the known SWS for a comparison purpose. The color bar is the acoustic radiation force-induced displacement in micrometer.

In Fig. 3.9, two-dimensional (2D) SWS image from Model 1 was generated by repeating the process as illustrated in Fig. 3.8b at different axial depths. The SW front arrival time and the estimated SWS images were shown in Figure 9b-c, respectively. In Fig. 3.9c, the estimated SWS was $1.32 \pm 0.14 \mathrm{~m} / \mathrm{s}$, similar to what was reported in the literature $1.3 \pm 0.44 \mathrm{~m} / \mathrm{s}$ [50]. Qualitatively, the displacement time-to-peak (TTP) image (Fig. 3.9b) estimated from simulated time-resolved displacement curves was comparable to the one (Fig. 3.9d) experimentally obtained. 

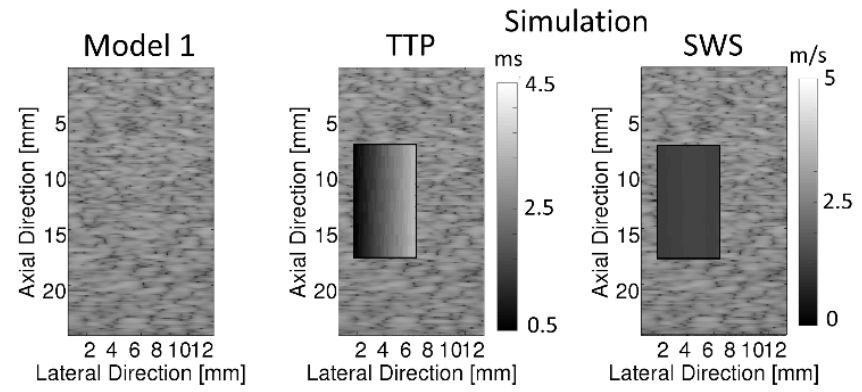

(a)

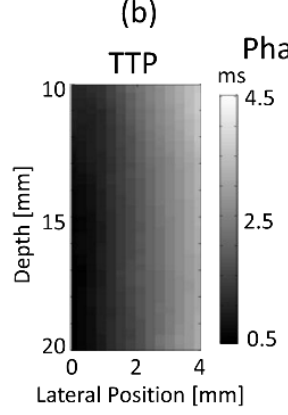

(d)

(c)

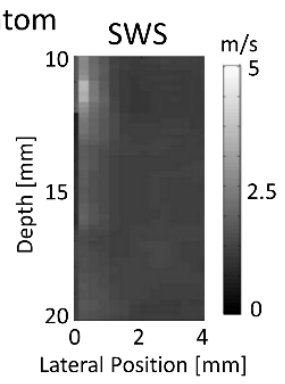

(e)

Figure 3.9. Two-dimensional (2D) SWS image estimated from Model 1 by using pSWE: (a) a simulated B-model image, (b) SW wave front arrival time within the rectangular ROI $(10 \mathrm{~mm} \times 6.5 \mathrm{~mm})$ and (c) estimated SWS within the same ROI $(10 \mathrm{~mm} \times 6.5$ $\mathrm{mm}) .(\mathrm{d})$ and (e) are experimental results available in the literature [44]. The unit of color bars in (b), (d) is in millisecond, while the unit of the color bars is m/s. TTP stands for time-to-peak.

\subsection{Biases in Virtual pSWE}

Estimated SWS values using wave data simulated by the virtual pSWE at two different depths are shown in Fig. 3.10. At each depth, three different transmit aperture sizes were simulated. Simulation results clearly exhibited that the SWS measurements may be dependent on the aperture size and focal depth, thereby resulting in biases in SWS measurements. Our simulation results (see Fig. 3.10a) are similar to those experimentally measured (see mean values in Fig. 3.10b) under the same condition. The differences between our simulation results and experimental results (Fig. 3.10a vs. mean values in Fig. 3.10b) were small (around $0.02 \mathrm{~m} / \mathrm{s}$ or $1.5 \%$ ). 


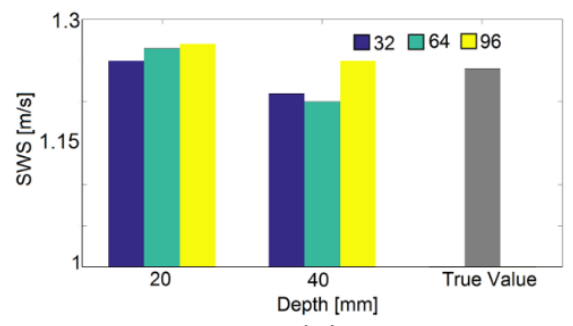

(a)

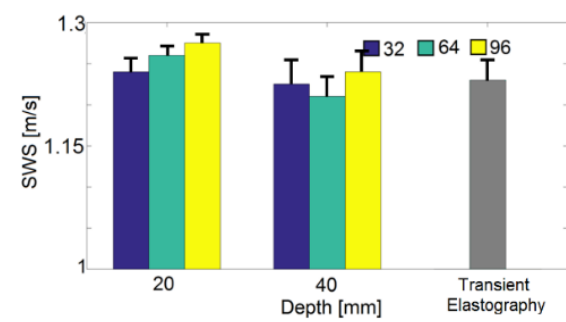

(b)

Figure 3.10. Plots of SWS measured at two focal depths (i.e. $20 \mathrm{~mm}$ and $40 \mathrm{~mm}$ ): (a) simulation results by the virtual pSWE and (b) experimental results by Zhao et al. At each depth, 3 different (transmit) aperture sizes (32, 64, and 96 elements) were used for the simulated push beam transmission. The experimental results were obtained from the original publication by Zhao et al. [18] Error bars in (b) represent one standard deviation.

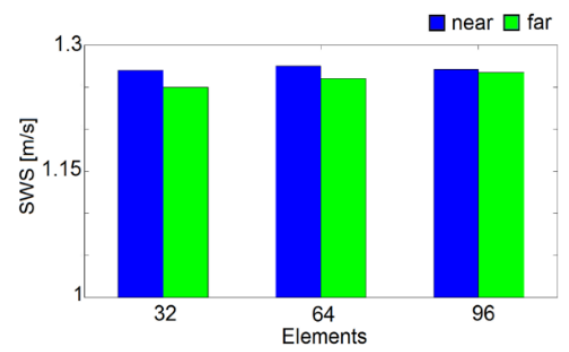

(a)

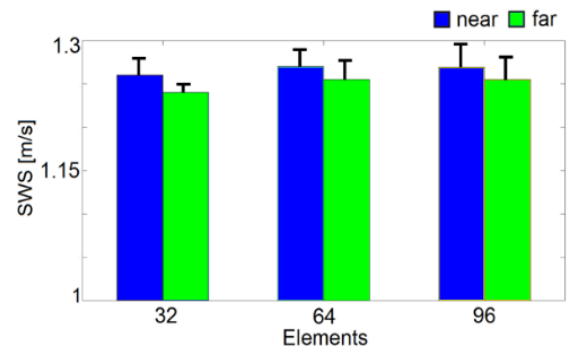

(b)

Figure 3.11. Plots of SWS measured at the near and far lateral tracking regions: (a) simulation results by the virtual pSWE and (b) experimental results by Zhao et al. The push beam was focused at $20 \mathrm{~mm}$ and 3 different (transmit) aperture sizes (32, 64, and 96 elements) were used for the simulated push beam transmission. The experimental results were obtained from the original publication by Zhao et al. [18] Error bars in (b) represent one standard deviation.

How the estimated SWS values were affected by the lateral tracking ranges are shown in Fig. 3.11. Both the simulation and experimental results showed that the estimated SWS values were higher in the near (lateral) tracking range than those estimated in the far (lateral) tracking range. Again, the differences between our simulation results and 
experimental results (Fig. 3.11a vs. mean values in Fig. 3.11b) were small (around 0.02 $\mathrm{m} / \mathrm{s}$ or $1.5 \%)$.

\subsection{Virtual SSI}

Virtual SSI was performed for Models 2 and 3. Results are summarized in Figs. 3.12-3.13. "Mach cone-shaped" plane shear waves [85] were observed in both models. Our results also confirmed that the angle of the plane shear wave was proportional to the ratio between the SWS and the speed of the moving supersonic source as suggested in the literature [85]. Visually, the SW propagation both in Models 2 and 3 was progressively distorted by heterogeneities (Fig. 3.12b) and such distortions had been accelerated after the SW wave fronts passed through respective harder inclusion (see Figs. 3.12c and 3.13c). It is worth noting that the modulus ratio between the background and the inclusion was higher for Model $3(1: 10)$, as compared to that in Model 2 (1:4). That explains why the (shear) wave interference patterns in Model 3 were clearly noticeable and visually more consistent with the shapes of the inclusion (see Fig. 3.13c).

Figs. 3.12d and 3.13d show the estimated SWS values as images in Models 2 and 3, respectively. We found that, due to tissue heterogeneities in Models 2 and 3, estimated SWS values from the background were less uniform and the relative variances of estimated SWS from the background increased to 4\% (Figure 2.26d) and 12.5\% (Figure 2.27d) for Models 2 and 3, respectively. Now referring to the inclusion in Model 2, the average estimated SWS was $4.05 \mathrm{~m} / \mathrm{s}$ and therefore it was underestimated by $9.4 \%$ as compared to the known SWS of $4.47 \mathrm{~m} / \mathrm{s}$. Similarly, the respective known SWS values were also estimated by $\sqrt{G / \rho}$ based on its predetermined material properties of Models 2 and 3 (see 
Table 2), where $G$ and $\rho$ are shear modulus and the mass density, respectively. Qualitatively, the inclusion shape in Figure 2.26d was similar to the shape experimentally obtained (see Figure 2.26e) in a tissue-mimicking phantom [83].

In Model 3, the mismatch between the fibro-glandular background and the simulated breast lesion was greater (1:10) and the tissue composition was more complex (Fig. 3.13a). Therefore, the reconstructed shape of the inclusion in Model 3 slightly deviated away from the known size and shape. Notably, there is a slight divot artifact in the reconstructed SWS image as shown in Fig. 3.13d. The same divot was also reported in the literature [83]. Although boundaries of the simulated breast lesion are not well visualized due to the presence of the "divot" artifact, a stiff region in the center of the SWS image well correlated with the simulated breast lesion can be clearly observed. The result shown in Fig. 3.13d is qualitatively similar to what were reported by others [50].

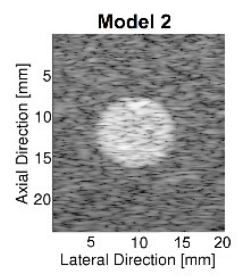

(a)

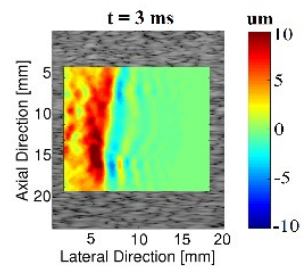

(b)

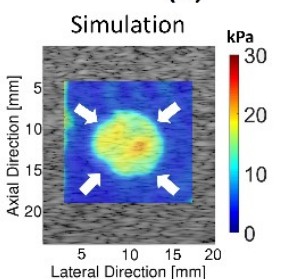

(d)

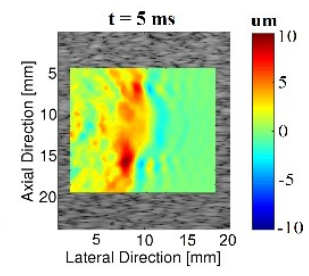

(c)

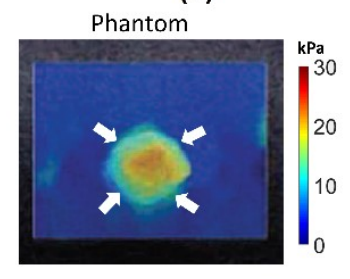

(e)

Figure 3.12. Results of virtual SSI for Model 2: (a) a simulated B-model image, simulated displacement images at (b) 3 milliseconds and (c) 5 milliseconds after the first pushing pulse started, (d) an image of estimated shear modulus (in $\mathrm{kPa}$ ) and (e) an image of experimentally estimated shear modulus (in $\mathrm{kPa}$ ) image in a tissue-mimicking phantom [83]. White arrows in (d) and (e) point to the reconstructed inclusion. The color 
bars in (b) and (c) are in micrometers. (b) and (c) represent the acoustic radiation forceinduced displacements at $t=3$ and 5 milliseconds.

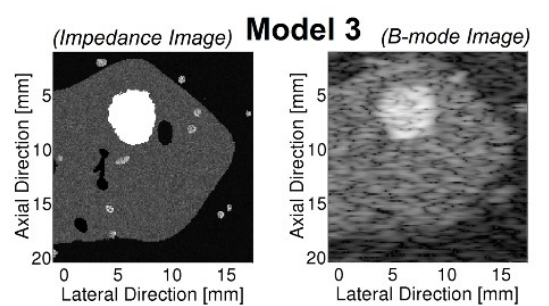

(a)

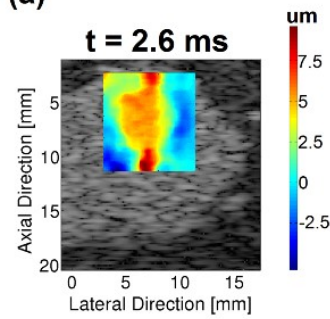

(c)

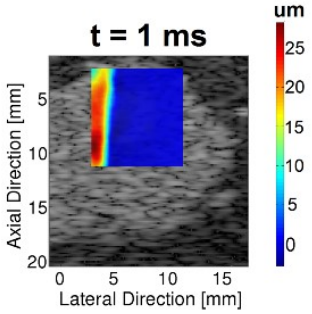

(b)

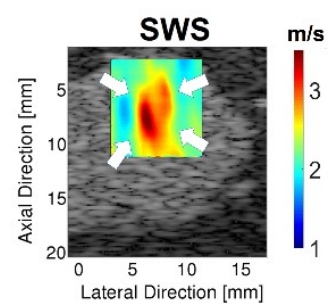

(d)

Figure 3.13. Results of virtual SSI for Model 3: (a) simulated impedance (left) and Bmodel (right) images, simulated displacement images at (b) 1 milliseconds and (c) 2.6 milliseconds after the first pushing pulse started, and (d) an image of estimated SWS. White arrows in (d) point to the reconstructed breast lesion using estimated SWS values. The color bars in (b) and (c) are in micrometer; (b) and (c) show the acoustic radiation force-induced displacements. The color bar in (d) represents the SWS in $\mathrm{m} / \mathrm{s}$.

\subsection{Virtual ARFI Imaging}

For visualization purposes, both peak displacement and (displacement) time-topeak (TTP) images were normalized between 0 and 1. Normalized ARFI peakdisplacement and (displacement) TTP images were simulated in Model 2 and Model 3, as shown in Figs. 3.14 and 3.15. Albeit slight differences, the respective inclusion (lesion) size appeared in the ARFI image was visually the same as what was observed in the respective B-mode image. Clearly, hard inclusions were displaced less than their surroundings. Furthermore, it took less time for hard inclusions to reach their peak 
displacements (see Figs. 3.14c and 3.15c). Estimated ARFI parameters - peak displacement and (displacement) TTP were relatively uniform and their relative variances were approximately 10\% (Figs. 3.14b-c and 3.15b-c).

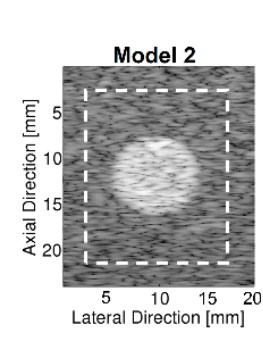

(a)

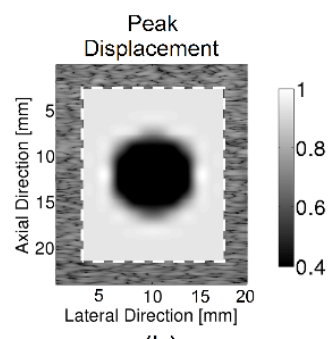

(b)

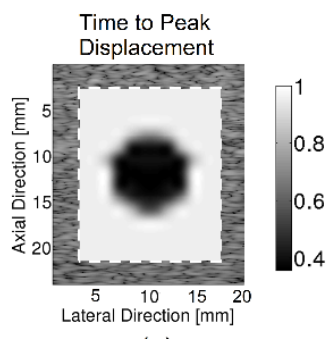

(c)

Figure 3.14. Results of virtual ARFI for Model 2: (a) a simulated B-model image, (b) simulated normalized peak displacement image and (c) simulated normalized (displacement) TTP image. The color bars are unit-less.

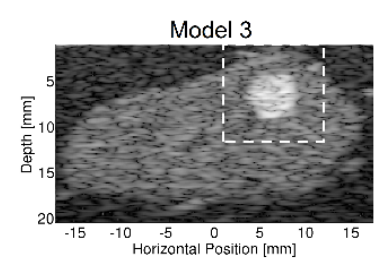

(a)

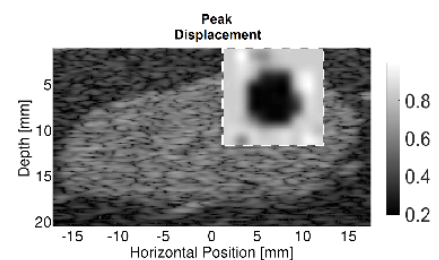

(b)

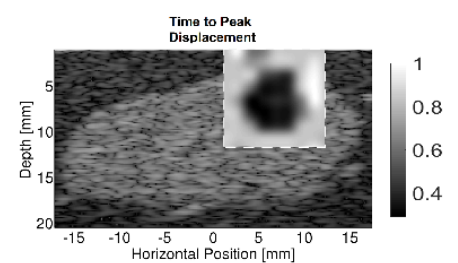

(c)

Figure 3.15. Results of virtual ARFI for Model 3: (a) a simulated B-model image, (b) simulated normalized peak displacement image and (c) simulated normalized (displacement) TTP image. The color bars are unit-less.

\subsection{Elastic Contrast (EC) Evaluation}

In Models 2 and 3, elastic contrast (EC) values (Eqn. 1) were estimated for three virtual imaging modalities using Methods A and B (see Fig. 3.7): SSI, ARFI, and SE. Results are listed below in Table 3.3. Overall, SSI shows the largest elastic contrast as compared with the other two qualitative methods — SE and ARFI images. 
Table 3.3. Tabulated results of elastic contrast (EC) values calculated from three virtual elastography methods. The SSI measurements were converted to elastic shear modulus values using $\sqrt{G / \rho}$, where $G$ and $\rho$ are shear modulus and the mass density, respectively. The underlying elastic contrast values were calculated based on data shown in Table 2 .

\begin{tabular}{ccccc}
\hline \hline & \multicolumn{2}{c}{ Model 2 } & \multicolumn{2}{c}{ Model 3 } \\
\cline { 2 - 5 } & Method A & Method B & Method A & Method B \\
\hline Underlying EC & 4 & 4 & 10 & 10 \\
SE & 2.32 & 2.12 & 1.92 & 1.72 \\
SSI & 3.53 & 3.23 & 3.35 & 3.19 \\
ARFI peak-displacement & 2.78 & 2.67 & 2.88 & 2.63 \\
ARFI (displacement) TTP & 3.22 & 3.15 & 3.20 & 2.91 \\
\hline \hline
\end{tabular}

\subsection{Discussion}

The proposed system leveraged other open-source/freeware packages, namely VTK, Tetgen [59], FEBio [81] and Field II [80]. All four software packages have been validated and widely used in biomedical research. Capitalizing on successes of these four existing packages, we demonstrated that a virtual acoustic radiation force-based elastography simulation platform can be implemented. The proposed acoustic radiation force-based elastography simulation platform is an extension of our previous work on virtual quasi-static SE simulation platform [84].

Simulated results by the proposed virtual elastography system in this feasibility study were quantitatively and qualitatively compared to tissue-mimicking and numerical phantom experiments reported in the literature $[50,85,89]$. Overall, we found that our virtual elastographic results were consistent with those published experimental results $[50$, 85, 89] as briefly summarized below. In the uniform numerical phantom (Model 1), the 
SWS speed estimated from virtual ultrasound simulations was essentially the same as the SWS speed experimentally measured $(1.32 \pm 0.14 \mathrm{~m} / \mathrm{s}$ vs. $1.3 \pm 0.44 \mathrm{~m} / \mathrm{s})$. However, both our virtual simulated and experimentally measured SWS values were slightly higher than the theoretical SWS $(1.26 \mathrm{~m} / \mathrm{s}$ as calculated by $\sqrt{G / \rho})$. Visually, the simulated distribution of (displacement) TTP was also comparable to what was experimentally measured (Fig. 9b vs. Fig. 9d). Work by Zhao et al. [18] demonstrated in tissue-mimicking materials that the SWS measurements were dependent on the focal depth, aperture size and lateral tracking range. Using the virtual pSWE, our simulation results in Model 1 (Fig. 3.10 and 3.11) could quantitatively reproduce those results by Zhao et al. In the simple inclusion phantom (Model 2), shear modulus values within the hard inclusion estimated by the proposed virtual SSI, on average, were approximately $10 \%$ lower than what were experimentally reported by Deffieux et al. [83] through visual inspections (see Fig. 10d vs. Fig. 10e), and were $17 \%$ lower than the underlying shear modulus value (see Table 3.2). Palmeri also reported [50] a similar finding. They found that such an underestimation increased as the stiffness values between inclusion and background further differed. The proposed virtual SSI confirmed their finding. We recognize that more rigorous validations and developments are still needed to ensure soundness of the proposed virtual elastography system. 


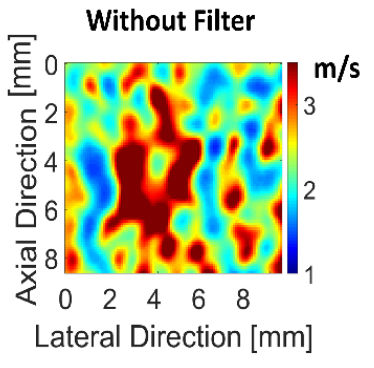

(a)

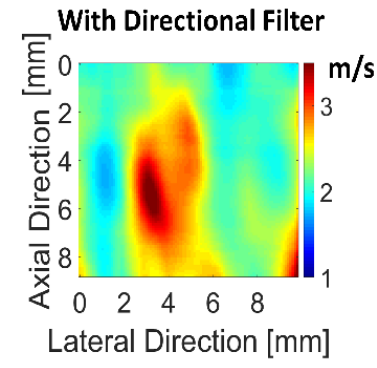

(b)

Figure 3.16. Two virtual SWS images comparing effects (a) without and (b) with the directional filter being applied. Results were based on Model 3 under the same condition by which all images in Figure 13 were obtained. The color bars represent the SWS in $\mathrm{m} / \mathrm{s}$.

The outcome of virtual SSI may also be dependent on signal processing techniques used to estimate SWS. One example is shown in Fig. 3.16 to exhibit the SWS reconstruction results with and without the directional filter. Fig. $\mathbf{3 . 1 6}$ demonstrated that quantitative differences may be introduced by different data processing strategies. Particularly, in the complex breast model (Model 3), when the directional filter [83] was not applied, the SWS image was quite noisy and the "divot artifact" in the estimated SW images was fairly pronounced (see Fig. 3.16a). After the directional filter had been applied, the SWS image became smoother though the "divot artifact" was slightly visible. The "divot artifact" was largely caused by changes in the SW morphology due to reflections at the stiff-to-soft and soft-to-stiff interface as the shear wave propagates through the boundary [50]. Evidently, SW distortions caused by tissue heterogeneities were clearly observed in our results (Fig. 3.13d).

As reported by Quantitative Imaging Biomarker Alliance [19], there were statistically significant differences among tissue-mimicking phantom results when 
different SWE systems were used. Those quantitative differences could partially be attributed to the fact that different SWE systems could have used different ultrasound system parameters and data/signal processing strategies. Preliminary results (Figures 10 and 11) showed that the proposed virtual elastography system can reproduce systemdependent SWS measurements. Consequently, the proposed system might be used to further investigate how (ultrasound) system parameters may affect SWS measurements. Since public releasing of those ultrasound system parameters and data/signal processing strategies by vendors is not feasible, performing virtual elastography simulations in heterogeneous, complex but known (viscoelastic) media may enable us to advance understanding of elastographic measurements in complex media, thereby aiding in the interpretation of clinical SSI results. Furthermore, this is an open-source development so that this process can be transparently done. Source codes of this virtual simulation platform will be made available from Github (https://github.com/jjiang-mtu/virtual-breast-project).

In this study, virtual breast tissues including tumors were assumed to be linearly elastic solids as a first approximation. Our preliminary results (see Table 3.3) suggested that three different modalities (SE, SSI, and ARFI) provided different image contrast values in Models 2 and 3. Particularly, results in Table 3 suggested that SSI offers the highest elastic contrast values as compared to SE and ARFI. This observation is consistent with the tissue-mimicking phantom results reported by Palmeri and Nightingale [16] and Peng et al. [93]. In the future, viscoelastic tissue properties will be included in this virtual elastography platform. Once the viscoelasticity is included, the proposed virtual system 
could also be applied to further investigate elastographic contrast and image resolution in heterogeneous and complex media.

Tests in ultrasound elastography are often done using human trials and tissuemimicking phantoms. Human trials often associate with increasingly high costs [37], while developments of realistic tissue-mimicking breast phantoms that can resemble the complexity of human organs and tissue properties remain as a significant challenge $[38,39]$. In this study, we suggest that the proposed virtual ultrasound elastography simulation platform could be used as an alternative. Given more developments and validations, the proposed platform can enable imaging physicists/scientists to perform nearly all ultrasound elastography simulations using a single gateway. This is important because performing advanced ultrasound elastography simulations requires not only interdisciplinary knowledge in acoustic physics, FEA, tissue mechanics, computer-aided design and image/signal analysis but also a substantial effort to fill gaps among multiple (commercial or free) software tools. This single gateway software platform could potentially lift this burden away from image physicists/scientists given further developments. Consequently, research efforts can be more steered toward improving our understanding of ultrasound elastography using complex, heterogeneous but known media that emulating in vivo breast anatomy. It is our vision that rigorous imaging tests of this kind can be conducted with transparency due to their open-source nature. The proposed system can also be used to accelerate development of new elastographic methods by using the concept of virtual prototyping. 
Furthermore, Zhao et al. [18] have also advocated an option to correct system parameter-dependent SWS measurements using computer simulations. The proposed virtual simulation platform seems a good tool for this. A correction table generated through the proposed virtual simulation platform involving realistic anatomy and material properties can be used to compensate for biases due to (ultrasound) system parameterdependency.

\subsection{Conclusion}

A virtual simulation platform for acoustic radiation force-based breast elastography was extended from an ultrasound quasi-static breast elastography simulation platform described in chapter 2 . The proposed virtual breast elastography system in this chapter inherited four key components from chapter 2: an ultrasound simulator (Field II), a mesh generator (Tetgen), a finite element solver (FEBio) and a visualization and data processing package (VTK). Using a simple message passing mechanism, functionalities have now been extended to acoustic radiation force-based elastography simulations. To show the ability of the proposed simulation platform, acoustic radiation force Impulse (ARFI) imaging, point shear wave elastography (pSWE) and supersonic shear imaging (SSI) were simulated. Our initial results were consistent with our expectations and what have been reported in the literature. The proposed (open-source) simulation platform can serve as a single gateway to perform many elastographic simulations in a transparent manner, thereby promoting collaborative developments. 


\section{Chapter 4. Statistical analysis of SWE measurements in random two-phase media $^{3}$}

SWE is increasingly being used to noninvasively stage liver fibrosis by measuring shear wave speed (SWS). Chapter 4 is to quantitatively investigate intrinsic variations among SWS measurements obtained from heterogeneous media such as fibrotic livers. This chapter demonstrated that the intrinsic variations in the SWS measurements, in general, follow a non-Gaussian distribution and are related to heterogeneous nature of the medium being measured. The non-Gaussian distribution was derived by the principle of Maximum Entropy (ME). Also, the performance of the derived distribution was evaluated using Monte-Carlo (MC) simulated shear wave data and against three other commonly used distribution.

\subsection{Derivation of Probability Density Function (PDF)}

Consider an elastic (lossless) heterogeneous medium, which consists of multiple isotropic non-overlapping phases with elastic bulk moduli $\left(K_{i}\right)$, shear moduli $\left(G_{i}\right)$, mass density values $\left(\rho_{i}\right)$ and the volume fractions of each phase $\left(c_{i} ; \sum c_{i}=1\right)$. The subscripts $i$ denotes phase index, respectively. For simplicity, the multi-phase medium can be effectively treated as isotropic and macroscopically homogeneous. Therefore, this medium which can be characterized by effective bulk modulus $(K)$, effective shear modulus $(G)$ and effective mass density $(\rho)$. Consequently, given a medium, $K$ and $G$ are two unknown random variables. Among most biological soft tissues, variations in mass density are small

\footnotetext{
${ }^{3}$ The material contained was previously published in Physics in Medicine and Biology, 2017 Jan;62(3):1149-1171. Refer Appendix G for granted permission to be republished.
} 
as compared to those in the shear modulus [90]. We treat the effective mass density $\rho$ as a constant to simplify the derivation.

Formally, we now assume that an arbitrary multi-phase medium can be specified by a set of physical properties $J=\left(K_{i}, G_{i}, \rho_{i}, c_{i}\right)$. Given the multi-phase medium whose properties can be specified by the set $J$, under the framework of effective medium theory, the macroscopic SWS of the medium $\left(V_{S}\right)$ can be related to the effective shear modulus $G$ by $[94,95]$,

$$
V_{s}=\sqrt{\frac{G}{\rho}}
$$

where $\rho$ is the effective mass density. Eqn. (4.1) is only valid with low-frequency shear wave propagation in elastic (lossless) media.

In information theory, Jaynes' information entropy $(H)$ [96] of the effective shear modulus $G$ is given below,

$$
H=-\int_{-\infty}^{+\infty} p(G) \ln \frac{p(G)}{m(G)} d G
$$

where $m(G)$ can be used to represent a prior information and $p(G)$ is a (histogram) distribution of $G$. Based on the principle of ME, the Jaynes' entropy $H$ reaches its maximum if $p(G)$ reflects the data distribution in the best possible way, given the prior information $m(G)$. Consequently, maximization of $H$ leads to an optimized $p(G)$ that best describes the given data $G$. In Eqn. (4.2), $m(G)$ has been set to 1, meaning that no prior information regarding the distribution of $G$ is available to us.

To ensure that the optimized $p(G)$ is a good fit of the given data $G$, the 60 
maximization of $H$ should be constrained by the statistical moments of the data $G$ as follows,

$$
\begin{aligned}
& \int_{-\infty}^{+\infty} p(G) d G=1 \\
& \int_{-\infty}^{+\infty} p(G) G d G=G_{E} \\
& \int_{-\infty}^{+\infty} p(G)\left(G-G_{E}\right)^{2} d G=G_{D}
\end{aligned}
$$

where $G_{E}$ and $G_{D}$ are mean and variance of the effective shear modulus $G$. It is important to note that $\mathrm{G}$ is a random variable and, now $p(G)$ becomes the PDF of the given effective modulus data $G$. A Lagrange multiplier method [97] was used to maximize $H$ in conjunction with Eqn. (4.1). This process yields an analytical expression of PDF of the macroscopic SWS $\left(V_{S}\right)$,

$$
p\left(V_{S}\right)=\frac{1}{\sqrt{2 \pi G_{D}}} e^{\frac{-\left(V_{S}^{2} \rho-G_{E}\right)^{2}}{2 G_{D}}} 2 \rho V_{S}
$$

A complete derivation of Eqn. (4.6) is provided in $\underline{\text { Appendix C. }}$.

\subsection{Model Evaluation}

The derived PDF, $p\left(V_{s}\right)$, was compared with three commonly used PDF functions (Section B.1) to demonstrate its fitness to simulated SWS data. As an integral part of this evaluation process, statistical model selection criteria (Section B.2) will be used as quantitative metrics to determine the performance.

\subsubsection{Commonly-Used PDFs}


Three commonly-used PDFs [98] (i.e. Gaussian distribution, log-normal distribution and generalized Gaussian distribution) were selected to compare with the theoretically-derived PDF (i.e. Eqn. (4.6)). The PDF of Gaussian distribution (GAD) can be written as follows,

$$
p^{G A D}\left(V_{S}\right)=\frac{1}{\sigma \sqrt{2 \pi}} e^{-\frac{\left(V_{S}-\mu\right)^{2}}{2 \sigma^{2}}}
$$

where $\mu$ and $\sigma$ are the mean and standard deviation of SWS, respectively.

The PDFs of log-normal distribution (LND) and generalized Gaussian distribution (GGD) are expressed as follows, respectively,

$$
\begin{aligned}
& p^{L N D}\left(V_{s}\right)=\frac{1}{V_{S} \sigma \sqrt{2 \pi}} e^{-\frac{\left(\ln V_{S}-\mu\right)^{2}}{2 \sigma^{2}}} \\
& p^{G G D}\left(V_{s}\right)=\frac{\beta}{2 \alpha \Gamma(1 / \beta)} e^{-\left(\left|V_{S}-\mu\right| / \alpha\right)^{\beta}}
\end{aligned}
$$

Similarly, in Eqn. (4.8), $\mu$ and $\sigma$ are the mean and standard deviation of $\ln \left(V_{s}\right)$, where $\ln (\quad)$ is a natural logarithm function, while $\mu$ is the mean of $V_{s}$ in Eqn. (4.9). In Eqn. (4.9), $\beta$ is estimated by using a Newton-Raphson iterative procedure [99]. Once $\beta$ is obtained, $\alpha$ can be evaluated as $\left(\frac{\beta}{N} \sum_{i=1}^{N}\left|V_{s i}-\mu\right|^{\beta}\right)^{\frac{1}{\beta}}$, where $N$ is the number of samples.

\subsubsection{Statistical Model Selection Criteria}

Two commonly used model-comparison criteria, namely the Akaike Information Criterion (AIC) [100] and the Bayesian Information Criterion (BIC) [101], were used to evaluate the relative quantity and fitness of the four statistical PDFs (i.e. Eqns. 4.6-4.9). 
Let $L=\prod_{i=1}^{n} p\left(V_{s i}\right)$ be the likelihood function of any of those four statistical PDFs $\left(p\left(V_{s i}\right)\right)$ and $k$ be the number of estimated parameters in the PDF. Then the AIC and the BIC of a given distribution can be, respectively, presented as follows,

$$
\begin{aligned}
& A I C=-2 \ln (L)+2 k \\
& B I C=-2 \ln (L)+k \cdot \ln (n)
\end{aligned}
$$

In general, when choosing from several models under consideration, the one with the lowest AIC and BIC is preferred in terms of data fitness.

\subsection{Overall Workflow of Numerical Experiments}

Data obtained from MC-simulated SW were generated to evaluate the derived PDF (see Eqn. (4.6)). As shown in Fig. 4.1, the corresponding MC simulations involve two steps. In the first step, one cycle of a low-frequency (i.e. $50 \mathrm{~Hz}, 100 \mathrm{~Hz}$ and $150 \mathrm{~Hz}$ ) plane [shear] wave excitation was prescribed and subsequently, SW was induced. Excitations with a single fixed frequency are similar to ones used in TE. The chosen excitation frequency falls in the range of a typical SW frequency excited in a clinical setting: between $50 \mathrm{~Hz}$ and $200 \mathrm{~Hz}$ [1]. SW propagation was simulated by using K-Wave, an open-source wave simulator [102]. Details of K-Wave simulations including boundary conditions can be found in subsection E and $\underline{\text { Appendix D }}$ below. In the second step, a direction filter [103] was first applied to simulated particle velocity data. Then, a lateral time-to-peak (TTP) algorithm [44] was adopted to calculate SWS along the wave propagation direction at different depths based on "filtered" particle velocity data. The temporal sampling frequency of the recorded signals was approximately $100 \mathrm{kHz}$ [44]. 

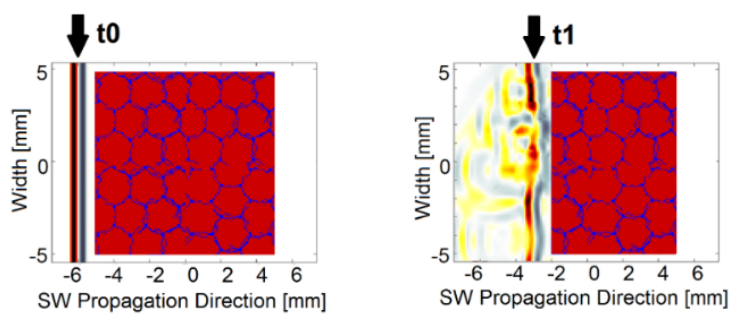

(a)

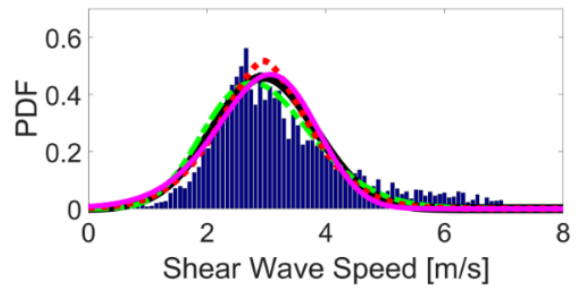

(b)

Figure 4.1. A workflow involving in the simulation of SW propagation through a stochastic two-phase medium using the K-Wave simulator: (a) a plot showing simulated SW that propagates through the medium downward and, (b) a histogram of estimated SWS values along the SW propagation direction, along with four fitted PDFs.

\subsection{Two-phase Fibrotic Liver Tissue Model}

The proposed two-phase stochastic tissue model was designated to mimic histological characteristics of liver fibrosis from two important aspects: (1) the amount of fibrosis and (2) architectural changes due to fibrosis [104]. This was done under the framework of METAVIR classification [105]. A schematic illustration of the proposed stochastic tissue model can be seen in Fig. 4.2a, where hepatocytes were arranged in lobules and each lobule was modeled as a hexagon. Each edge of those hexagons was approximately $1 \mathrm{~mm}$ [106]. Each septum, forming an edge of a lobule, was connected by portal triads. In the early stages of liver fibrosis (i.e. METAVIR scores F1 and F2), liver injuries occur and inflammatory cells migrate across endothelium of portal vessels. This process will initiate depositions of collagen. Thus, in the early stages, collagen will be 
deposited around portal tracts with extensions into adjacent peri-portal regions along septa, as shown in Figs. 4.3b-c and those collagen depositions look like isolated islands (See Figs. 4.3b-c). As the liver fibrosis progresses (i.e. METAVIR scores F3 and F4), inflammatory cells migrating across the endothelium of hepatic sinusoids can gain direct access to the liver parenchyma, thereby causing lobular inflammation. Consequently, fibrous expansions of most portal areas cause portal-to-portal bridging or portal-to-central bridging [107], as shown in Figs. 4.3d-e. In other words, at the late stages of the liver fibrosis, deposited collagen "islands" (see Figures 3(b)-(c)) have been connected by septa and the overall architectural appearance looks more like a complex web (see Fig. 4.3e). Overall, the proposed stochastic tissue model captures both the increasing presence of collagen content and spatial characteristics of collagen depositions, as shown in Figs. 4.3be.

An in-house MATLAB script (Mathworks Inc., MA, USA) was used to create tissue compositions that represent different fibrosis stages, given a volume fraction of collagen content. The selected volume fractions of collagen content were adopted based on measured values of collagen proportional area (CPA), as shown in Table 4.1 [108]. All necessary parameters needed for the simulations of SW propagation were based on data available in the literature. More specifically, shear moduli of the background (i.e. normal liver parenchyma) and the fibrotic inclusions (i.e. collagen) were set to $3.24 \mathrm{kPa}$ (SWS = $1.8 \mathrm{~m} / \mathrm{s})$ [109] and $400 \mathrm{kPa}(\mathrm{SWS}=20 \mathrm{~m} / \mathrm{s})[110,111]$, respectively. The mass density of the background and the fibrotic inclusions were set to be $1000 \mathrm{~kg} / \mathrm{m}^{3}$ and $1200 \mathrm{~kg} / \mathrm{m}^{3}$ [90], respectively. The compressional sound speed of the background and the fibrotic 
inclusions were set to be $1540 \mathrm{~m} / \mathrm{s}$ and $2000 \mathrm{~m} / \mathrm{s}$ [90], respectively.

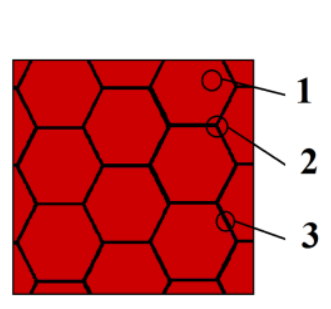

(a)

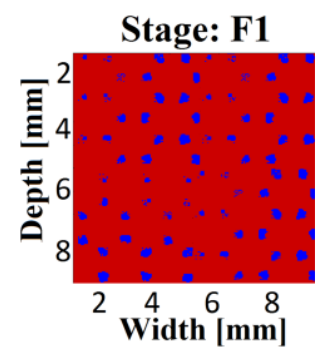

(b)

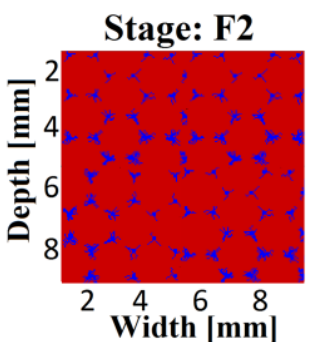

(c)

\section{Hepatocytes \\ 2. Portal Triad \\ 3. Septa}

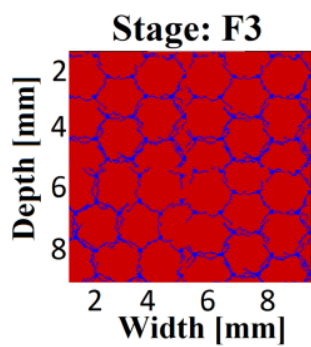

(d)

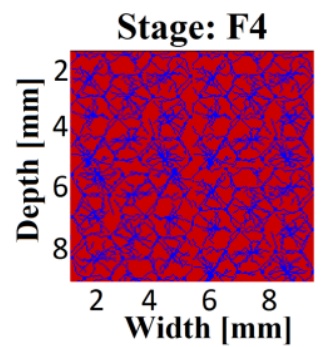

(e)

Figure 4.2. (a) a 2D illustration of liver anatomy; (b) Stage F1 with 8\% collagenous tissue; (c) Stage F2 with $8 \%$ collagenous tissue; (d) Stage F3 with 10\% collagenous tissues; and (e) Stage F4 with 25\% collagenous tissues. In (b)-(e), the red is normal liver tissue and the blue is collagenous connective tissue. Illustrations of fibrosis from F1 to F4 were based on the METAVIR classifications [105].

Table 4.1 Collagen proportional area (CPA) for 4 different liver fibrosis stages (F1-F4).

Data were taken from reference [108].

\begin{tabular}{cccccc}
$\begin{array}{c}\text { METAVI } \\
\text { R }\end{array}$ & F0 & F1 & F2 & F3 & F4 \\
stage & & & & & \\
\hline \hline CPA & $2.2 \sim$ & $1.8 \sim$ & $2.26 \sim$ & $4.96 \sim$ & $7.8 \sim$ \\
& $9.67 \%$ & $15.97 \%$ & $16.68 \%$ & $20.73 \%$ & $32.67 \%$ \\
\hline
\end{tabular}

In order to assess sizes of those inclusions with complex shapes (see Fig. 4.3(b)(e)), correlation lengths were calculated based on two-point correlation function [112]. 
Mathematically, a two-point correlation function $S\left(r_{1}, r_{2}\right)$ between two arbitrary points $p_{1}$ and $p_{2}$, is defined as follows,

$$
S\left(p_{1}, p_{2}\right)=<I\left(p_{1}\right) I\left(p_{2}\right)>
$$

where angular brackets denote an ensemble average and $I(p)$ is defined as

$$
I(p)=\left\{\begin{array}{c}
1, \text { if } p \text { is in the inclusion phase } \\
0, \text { otherwise }
\end{array}\right.
$$

Equation (4.12) represents a probability of finding two points $p_{1}$ and $p_{2}$ in the same phase separated by a distance $r$. Consequently, the correlation length is defined as the distance by which the correlation drops to a stable plateau.

\subsection{Performing 2D SW Simulations}

A uniform $90 \mathrm{~mm}$ (along the wave propagation direction) $\times 22 \mathrm{~mm}$ computing domain was used to generate MC simulated SW propagation data, as shown in Fig. 4.3. A $20 \mathrm{~mm} \times 20 \mathrm{~mm}$ central rectilinear region of interest (ROI) containing either $10 \%$ or $30 \%$ inclusions was embedded into the computing domain. The grid spacing within the entire computing domain was uniform and the pixel/grid size was $0.1-\mathrm{mm} \times 0.1-\mathrm{mm}$. Mesh sensitivity tests were performed to verify that results were not sensitive to the chosen grid size. Compression and SW velocity parameters (i.e. compressional sound speed, shear wave speed, and mass density) were assigned to each pixel in the rectilinear [computing] grid. In addition to material properties presented in subsection D of the Materials and Methods Section above, other key simulation details can be found in Table 4.2. One cycle of low-frequency (i.e. $50 \mathrm{~Hz}, 100 \mathrm{~Hz}$, and $150 \mathrm{~Hz}$ ) transient (up-down) vibration (see the green vertical line in Fig. 4.4) was prescribed to generate SW that propagates laterally (see 
the arrow in Fig. 4.4) for each realization. The particle motion (also known as the SW polarization direction) followed the up-down direction (Fig. 4.4) and was perpendicular to the SW propagation direction. The simulation duration was approximately 0.01 second to allow the induced SW to propagate through the entire ROI. Details and a brief justification of this computing domain model including boundary conditions can be found in $\underline{\text { Appendix }}$ $\underline{\mathbf{D}}$

Table 4.2 Simulation details of the stochastic tissue model using K-Wave. The correlation length was quantified using two-point correlation function [112].

\begin{tabular}{cccc}
\hline \hline CPA & $\begin{array}{c}\text { Number of } \\
\text { Realizations } \\
\text { (for each stage) }\end{array}$ & $\begin{array}{c}\text { Stage (correlation length) } \\
\text { (unit: } \mathrm{mm})\end{array}$ & $\begin{array}{c}\text { Number of SWS } \\
\text { measurements } \\
\text { (for each stage) }\end{array}$ \\
\hline $10 \%$ & 5 & F1 (0.5), F2 (0.6) & 11,000 \\
$30 \%$ & 5 & F3 $(1.1)$, F4 $(1.3)$ & 11,000 \\
\hline \hline
\end{tabular}

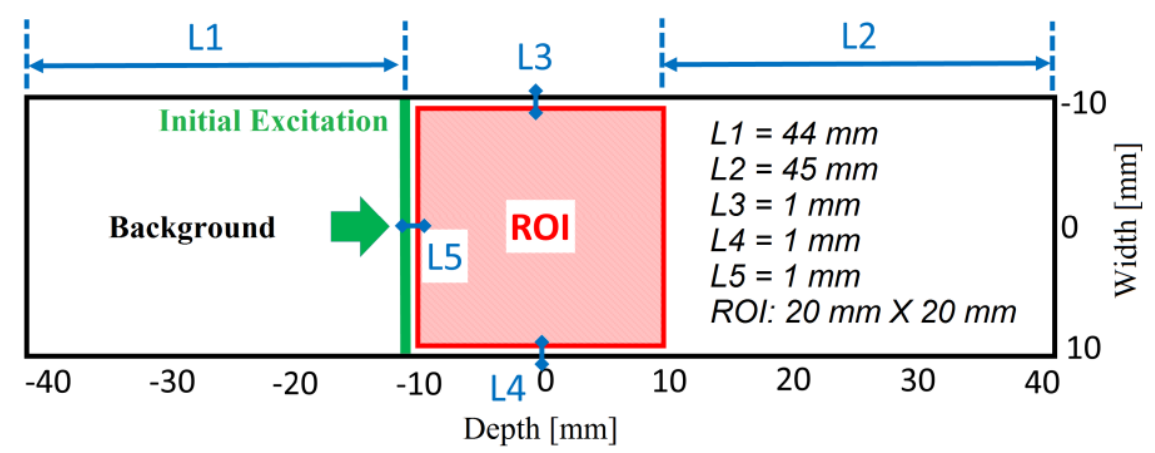

Figure 4.3. An illustration of computing domain used for all K-Wave simulations. A (20 $\mathrm{mm} \times 20 \mathrm{~mm}$ ) region of interest (ROI) containing either $10 \%$ or $30 \%$ inclusions was embedded into a large $(90 \mathrm{~mm} \times 22 \mathrm{~mm})$ uniform background. The green vertical line indicates the location of initial mechanical vibration, while the green arrow represents the SW propagation direction.

\subsection{Assessment of SWS Distributions}


To reduce memory usage in the calculations, only $49 \times 48(n \times \mathrm{q})$ time-resolved SWS curves obtained within the ROI (see Figure 4) were stored. In other words, we only stored simulated SW data from a coarsened uniform rectilinear grid whose grid spacing was $0.4 \times 0.4-\mathrm{mm}$, as shown in Fig. 4.4. The SWS at an arbitrary location $(m, p)$ will be estimated by detecting the time-delay $(\Delta t)$ between two time-resolved particle velocity curves located at $(m, p)$ and $(1, p)$, respectively, using a correlation-based method [44]. This process resulted in $2256(48 \times 47)$ SWS values for each simulated field. More specifically, SWS values were estimated at different width positions for each depth. Then, a 2D map of SWS values in the field of view can be obtained. PDF parameters were estimated by fitting simulated SWS data to respective equations (see Eqn. (4.6) - (4.9)). Only SWS data ranged from $5 \%$ to $95 \%$ quantiles were used.

In addition to analyses of all 11,000 SWS data together (see Table 4.2), we also randomly selected 100 data points from all 11,000 data points in order to mimic "clinical" SWS data. This process was repeated 100 times to generate 100 realizations of simulated "clinical" SWS data for each fibrosis stage.

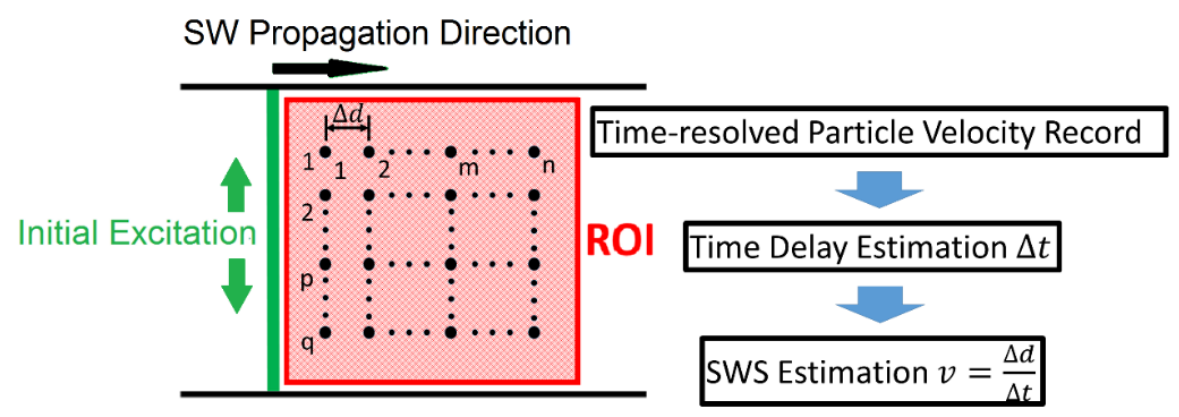

Figure 4.4. A schematic illustration of SWS estimation. The black arrows in the left figure represent the SW propagation direction. The time-resolved particle velocity curves were recorded for $\mathrm{n} \times \mathrm{q}$ locations (red dots in the left figure). Each red dot represents a time- 
resolved particle velocity curve in this illustration. The process of correlation-based shear wave speed estimation is shown in the right flowchart.

\subsection{Performance of The Derived PDF}

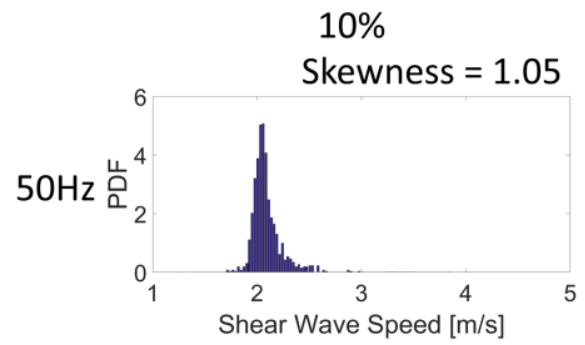

(a)

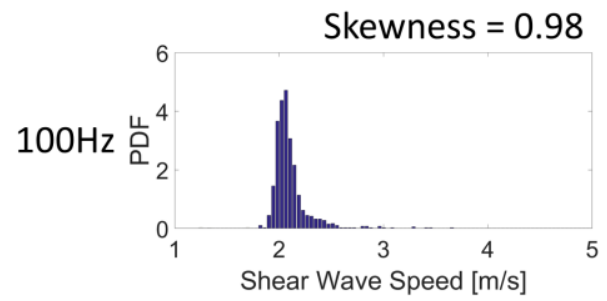

(c)

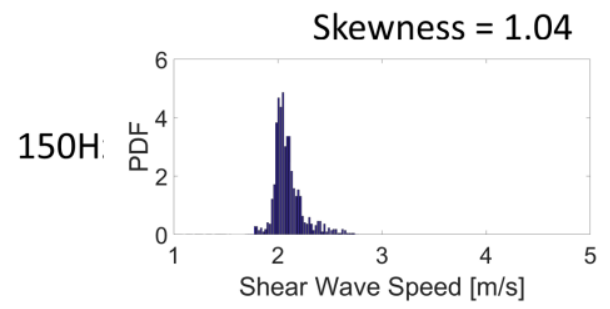

(e)

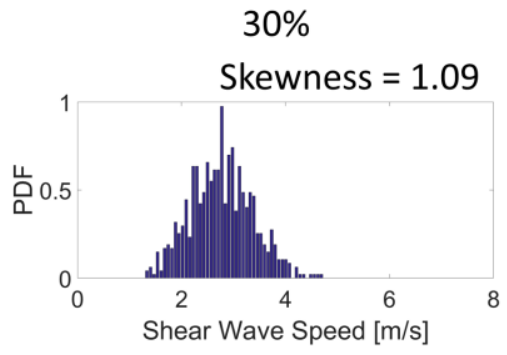

(b)

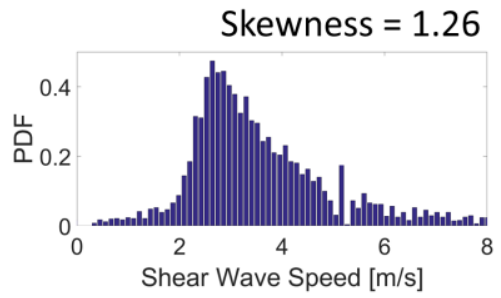

(d)

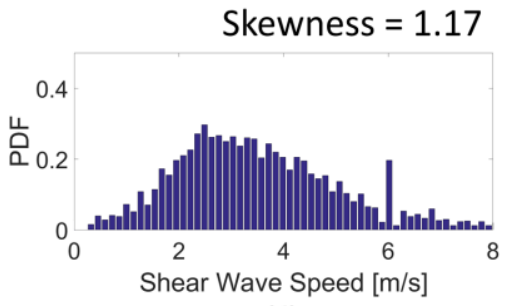

(f)

Figure 4.5. Histogram distributions of all M-C simulated SWS data from two different volume fractions $(10 \%$ and $30 \%)$. Simulation details were summarized in Table 2. Skewness was calculated for each histogram.

When histograms obtained from all 11,000 data points were examined, we found that the overall distribution was unsymmetrical as shown in Fig. 4.5. The skewness [113] was calculated. We found that consistent skewness values were obtained. In all six cases, 
the skewness was positive, denoting the SWS data were skewed toward the right. Of note, the skewness of any perfectly symmetric distribution (e.g. Gaussian distribution) is zero.

Representative simulated 'clinical' SWS data (i.e. 100 data points) are displayed in Fig. 4.6 as histograms. Relative AIC and BIC values were calculated from those simulated 'clinical' SWS data and their results were listed in Table 4.3. Relative AIC and BIC values were obtained by normalizing absolute AIC or BIC values (see Eqns. 10-11) obtained from other three PDF models by the respective AIC or BIC value from the derived PDF. Data in Table 4.3 indicated that the overall performance of the derived PDF performed is better than other three PDFs, though the relative differences of AIC and BIC among these four PDFs were small $(<3 \%)$. Results shown in Table 4.3 were consistent with the visual perception shown in Fig. 4.6.

Using simulated "clinical" SWS data, parameters estimated by the derived PDF, GAD and LND were converted to means and standard deviations of the underlying shear modulus values. Comparative results are shown in Table 4.4. When the excitation frequency was $50 \mathrm{~Hz}$, all four PDFs essentially gave the similar mean values of the fitted shear moduli. When the excitation frequency increased to $150 \mathrm{~Hz}$, the discrepancies becomes more noticeable. Particularly, at the $150 \mathrm{~Hz}$ and with $30 \%$ inclusions, mean values between the proposed PDF and the GAD differed by approximately $24 \%$. The difference between the proposed PDF and the LND increased to roughly $46 \%$ under the same condition. Results in Table 4.4 also suggested that standard deviations estimated using the proposed PDF were considerably lower than those obtained by GAD and LND for all cases investigated. 


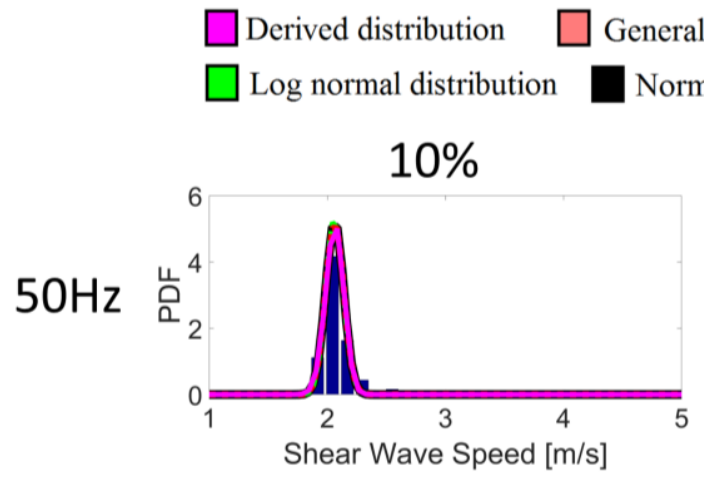

(a)

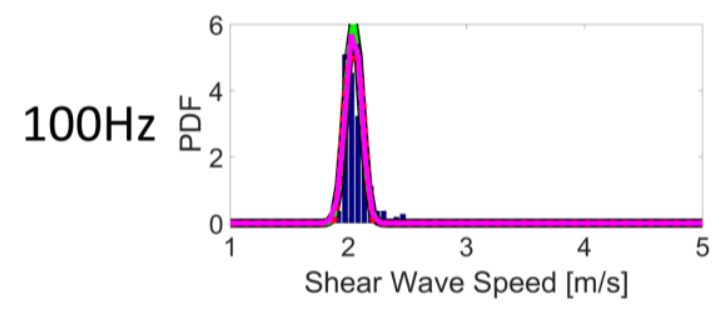

(c)

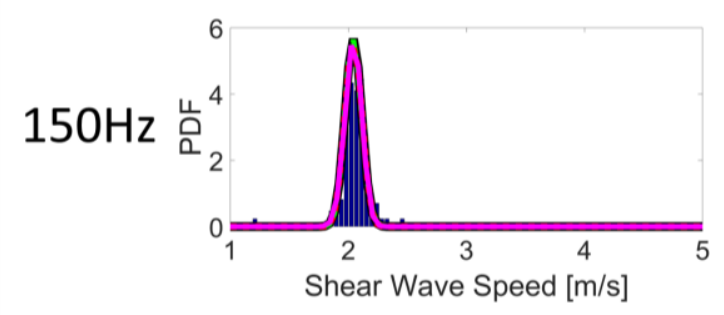

(e)

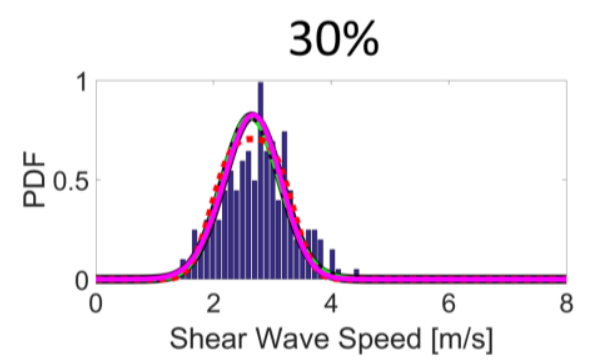

(b)

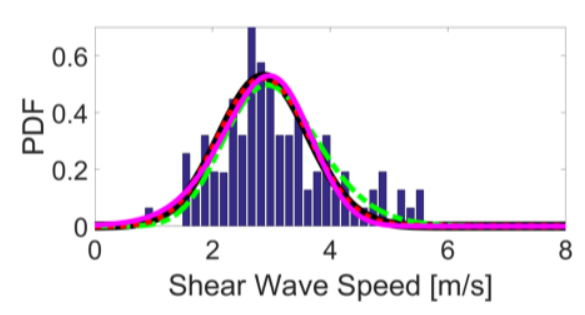

(d)

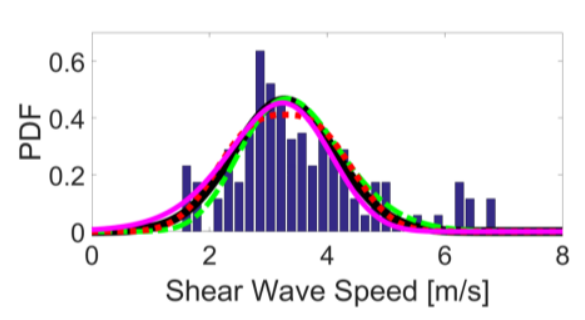

(f)

Figure 4.6. Representative histogram distributions of $\mathrm{M}-\mathrm{C}$ simulated "clinical" SWS data from two different volume fractions $(10 \%$ and $30 \%)$. Each histogram was generated using 100 data points and simulation details were summarized in Table 2. Four PDFs were overlaid with the histogram on each plot.

Table 4.3 Tabulated results about relative AIC and BIC values using 100 realizations of simulated 'clinical' data. Relative AIC and BIC values were obtained by normalizing absolute AIC or BIC values obtained from other three pdf models by the respective AIC or BIC value from the derived PDF. 


\begin{tabular}{|c|c|c|c|c|c|}
\hline \multirow[b]{2}{*}{$\begin{array}{c}\text { Inclusion } \\
\text { Volume } \\
\text { Fraction }\end{array}$} & & \multicolumn{2}{|c|}{ AIC } & \multicolumn{2}{|c|}{ BIC } \\
\hline & & $10 \%$ & $30 \%$ & $10 \%$ & $30 \%$ \\
\hline LND & \multirow{4}{*}{$50 \mathrm{~Hz}$} & -0.99 & 1.01 & -0.99 & 1.01 \\
\hline GGD & & -0.99 & 1.02 & -0.99 & 1.02 \\
\hline GAD & & -1 & 1.01 & -1 & 1.01 \\
\hline Derived PDF & & -1 & 1 & -1 & 1 \\
\hline LND & \multirow{4}{*}{$100 \mathrm{~Hz}$} & -0.97 & 1.02 & -0.97 & 1.02 \\
\hline GGD & & -1 & 1.01 & -1 & 1.01 \\
\hline GAD & & $\begin{array}{l}-0.99 \\
\end{array}$ & 1 & -0.99 & 1 \\
\hline Derived PDF & & -1 & 1 & -1 & 1 \\
\hline LND & \multirow{4}{*}{$150 \mathrm{~Hz}$} & -0.97 & 1.03 & -0.97 & 1.03 \\
\hline GGD & & -0.99 & 1.01 & -0.99 & 1.01 \\
\hline GAD & & -0.99 & 1 & -0.99 & 1 \\
\hline Derived PDF & & -1 & 1 & -1 & 1 \\
\hline
\end{tabular}

Table 4.4 Tabulated means and standard deviations (STD) of underlying shear modulus values using simulated "clinical" SWS data. Those parameters were estimated based on fitted four PDFs: the derived PDF, GAD, LND, and GGD, in two random media containing $10 \%$ and $30 \%$ inclusions, respectively. Of note, the standard deviation (STD) cannot be directly estimated using GGD (see Eqn. (4.9)).

\begin{tabular}{lccccccc}
\hline \hline & & \multicolumn{2}{c}{$\mathbf{5 0 H z}$} & \multicolumn{2}{c}{$\mathbf{1 0 0 H z}$} & \multicolumn{2}{c}{$\mathbf{1 5 0 H z}$} \\
\cline { 3 - 8 } & & $\begin{array}{c}\text { Mean } \\
(\mathbf{k P a})\end{array}$ & $\begin{array}{c}\text { STD } \\
(\mathbf{k P a})\end{array}$ & $\begin{array}{c}\text { Mean } \\
(\mathbf{k P a})\end{array}$ & $\begin{array}{c}\text { STD } \\
(\mathbf{k P a})\end{array}$ & $\begin{array}{c}\text { Mean } \\
(\mathbf{k P a})\end{array}$ & $\begin{array}{c}\text { STD } \\
(\mathbf{k P a})\end{array}$ \\
\hline \multirow{4}{*}{$\mathbf{1 0 \%}$} & Derived & 4.13 & 0.1 & 4.15 & 0.09 & 4.41 & 0.78 \\
& PDF & & & & & & \\
& GAD & 4.29 & 0.35 & 4.37 & 0.28 & 4.70 & 0.89 \\
& LND & 4.27 & 0.61 & 5.32 & 0.56 & 5.30 & 1.21 \\
& GGD & 4.28 & N/A & 4.29 & N/A & 4.84 & N/A \\
\cline { 3 - 8 } & Derived & 7.88 & 2.40 & 8.35 & 5.54 & 8.81 & 6.71 \\
$\mathbf{3 0 \%}$ & PDF & & & & & & \\
& GAD & 7.52 & 2.44 & 9.28 & 5.58 & 10.93 & 8.57 \\
& LND & 8.13 & 2.42 & 11.31 & 6.73 & 12.92 & 8.59 \\
\hline \hline & & & 73 & & & &
\end{tabular}




\begin{tabular}{lllllll} 
GGD & 7.92 & N/A & 8.46 & N/A & 9.01 & N/A \\
\hline \hline
\end{tabular}

We also found that, overall, the fluctuation of SWS decreased in all heterogeneous media as the traveling distance increased. In order to demonstrate that, we replot SWS values obtained from the excitation frequency of $150 \mathrm{~Hz}$ along the SW propagation direction in Fig. 4.7 below as an example.

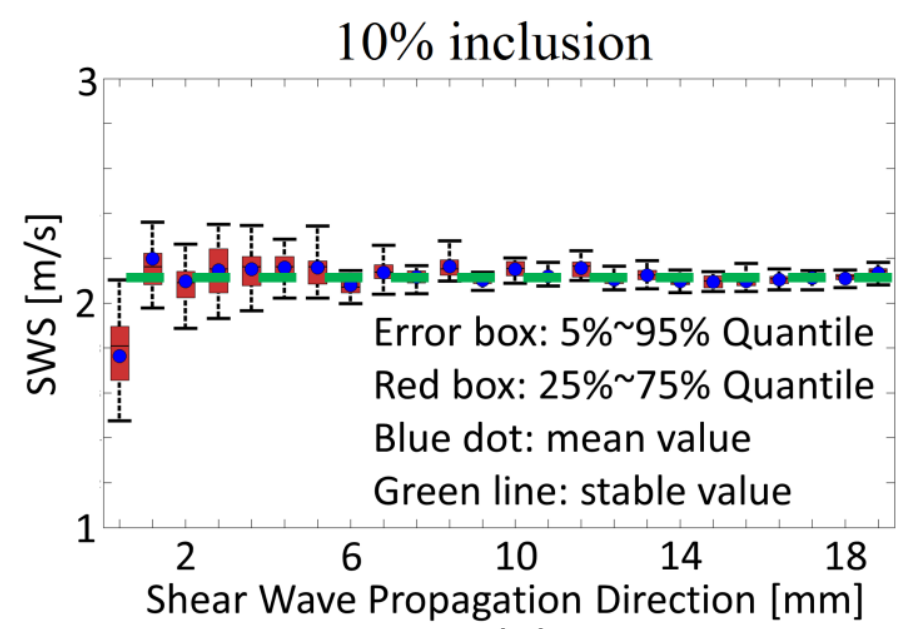

(a)

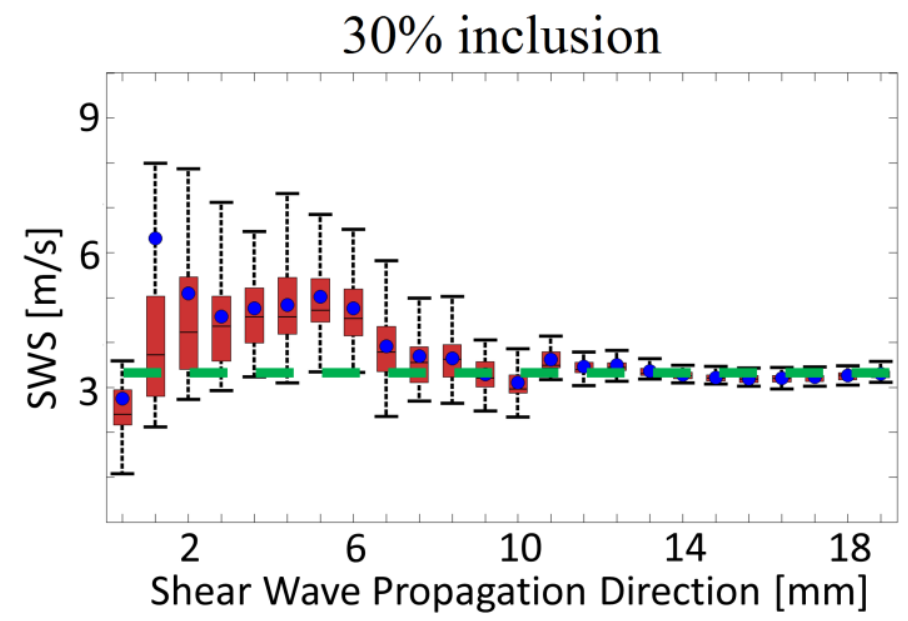

(b) 
Figure 4.7. Plots of SWS values along shear wave propagation direction (excitation frequency of $150 \mathrm{~Hz}$ ) based on all simulated SWS data. The green lines in (a) and (b) indicate respective mean values obtained through fitting of the derived PDF.

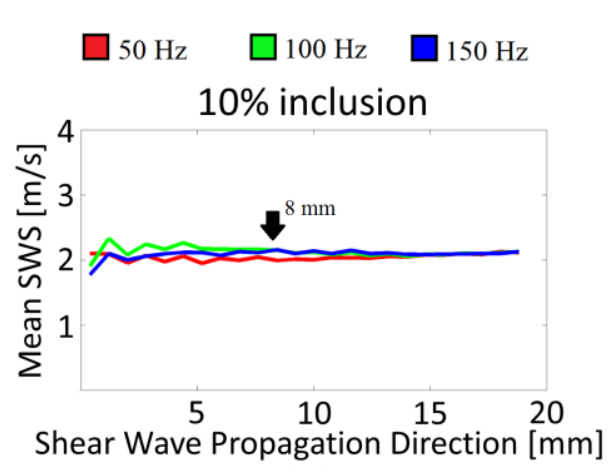

(a)

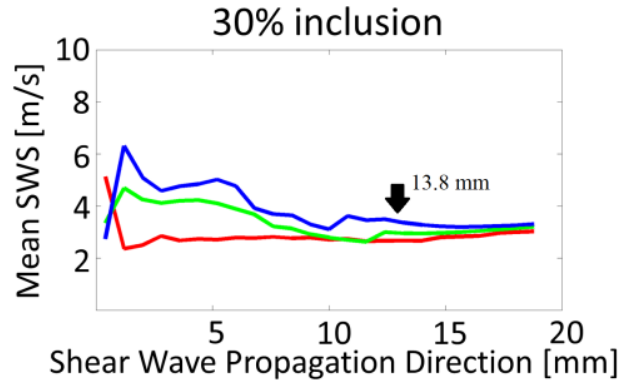

(b)

Figure 4.8. Plots of mean values of SWS along the shear wave propagation direction under two different volume fraction of inclusions (i.e. 10\%, 30\%). Each plot illustrates results obtained from three different $\mathrm{SW}$ frequencies $(50 \mathrm{~Hz}, 100 \mathrm{~Hz}$, and $150 \mathrm{~Hz})$. The black arrows represent the stabilization distance.

These results in Fig. 4.7 suggest that the SWS became stable (low variance and convergence to the mean SWS) after the SW travels a short distance. In the wave physics literature, the term "short" often referred to the fact the distances are on the same order as respective wavelengths. We assumed that the stabilization is achieved once the absolute difference between the instantaneous mean SWS (over multiple realizations) along SW propagation direction and the mean SWS through fitting the proposed PDF is less than 0.1 $\mathrm{m} / \mathrm{s}$. The traveling distance by which the wave stabilization is achieved is defined as the stabilization distance. As shown in Fig. 4.8, the required stabilization distance increased from $8 \mathrm{~mm}$ to $14 \mathrm{~mm}$ with the increase of heterogeneity from $10 \%$ of inclusions to $30 \%$ of inclusions. Also, the stabilization distance is not correlated to the excitation frequency, as 
shown in Fig. 4.8 below.

\subsection{Discussion}

One contribution of this work is that we found that the simulated SWS values in heterogeneous media have un-symmetric distributions (see Fig. 4.6). At the same time, through the principle of ME, the derived PDF (see Eqn. (6)) has an asymmetric form. Overall, the proposed PDF performed the best among all four PDFs (see Tables 4.3 and 4.4). It is also worth noting that the performance of GGD was close to that of the derived PDF. However, GGD has a complex form (i.e. Gamma function and three parameters; see in Eqn. (9)) and two parameters of GGD have no physical meanings. Consequently, the proposed PDF is advantageous in terms of both its solid theoretical basis and physically meaningful parameters. Certainly, this observation needs to be further investigated using in vivo biological data.

Results in Table 4.4 also indicated that different mean values of shear moduli, as well as different standard deviations, were obtained when different PDFs were used to fit the same data. The difference in terms of the mean shear modulus could be as high as $24 \%$ between the derived PDF and the Gaussian for the models that contain 30\% inclusions and were excited by $150 \mathrm{~Hz}$ perturbations. It was also found that such discrepancies increased as the percentage of fibrosis increased from $10 \%$ to $30 \%$, as shown in Table 4.4. This observation may have potential clinical implications. Because better thresholds/cut-off values can perhaps be determined for staging liver fibrosis if a better statistical PDF model can be adopted. As of now, the Gaussian Distribution PDF has been implicitly used [114, 115]. Moreover, two parameters (i.e. $G_{E}$ and $G_{D}$ ) of the derived PDF are the mean and 
variance of the underlying shear modulus of the stochastic medium. Certainly, more work is needed to demonstrate the proposed PDF is indeed advantageous.

The stochastic liver tissue model consisted of two components: normal liver tissues and (micro-packet of) fibrotic liver tissues. In the literature, normal liver shear modulus was measured to be 3.7-7.0 $\mathrm{kPa}$ in men and 3.3-6.8 $\mathrm{kPa}$ in women, respectively [109]. In this study, the normal liver shear modulus was set to be around $4 \mathrm{kPa}$ (SWS: $1.8 \mathrm{~m} / \mathrm{s}$ ) and the shear modulus value of fibrotic liver tissues was elevated to $400 \mathrm{kPa}$ (SWS: $20 \mathrm{~m} / \mathrm{s}$ ). Because, to our knowledge, there is no mechanical testing data available for fibrotic (collagenous) liver tissues at the spatial scale of 100 micrometers, the (micro-packet) of fibrotic tissues was chosen to be close to the shear modulus values of arterial tissues [110]. We have varied the shear modulus values of the fibrotic tissues from $900 \mathrm{kPa}$ (SWS: 30 $\mathrm{m} / \mathrm{s}$ ) to $2.5 \mathrm{MPa}$ (SWS: $50 \mathrm{~m} / \mathrm{s}$ ) and findings were not sensitive to the choice of the shear modulus values of the fibrotic tissues.

To obtain the M-C simulated SWS distribution, pointwise estimations of SWS were performed for each realization. The processing kernel length was $0.4 \mathrm{~mm}$. To verify the kernel length sensitivity for the simulated SWS distribution, our results were reprocessed using a processing kernel length of $1.2 \mathrm{~mm}$. We found that the means of SWS remained to be the same but the variances of SWS were typically reduced by approximately $5 \%$.

Our results shown in Figs. 4.8 and 4.9 also suggested that wave fronts with a plane wave propagating in a lossless (elastic) medium may be stabilized after certain stabilization distances, dependent on the nature of the heterogeneous random media. This finding is consistent with a proof in the literature of applied mathematics [116]. Intuitively, the 
relationship between the variations in SWS and the presence of inhomogeneity can be explained as follows. It is easy to see that the presence of inclusions leads to some distortions of wave fronts, resulting in variations among local SWS values. The degree of wave distortion is likely proportional to how heavily inclusions are presented along the wave propagation pathway. That is why variations in SWS were greater in more heterogeneous media.

Since the derivation of the PDF ignored the viscosity of soft tissue [117]. Thus, the derived PDF is valid when the SW propagates within lossless (elastic) media. In the future work, the viscosity-dependent dispersion will be considered. If verified in biological tissues, this observation may help us to determine optimal locations from which the SWS should be tracked by balancing the stabilization of wave fronts and attenuation of SW amplitudes. Furthermore, the stabilization distance was not proportional to the excitation frequency (Fig. 4.9). We stipulate that this observation is reasonable because the elastic wave scattering is within the Rayleigh Regime, given the wavelength (shear wave frequency: $50 \mathrm{~Hz}, 100 \mathrm{~Hz}$, and $150 \mathrm{~Hz}$ ). It is worth noting that estimated mean values of SWS (Table 4.4) under three excitation frequencies slightly differed (approximately 2$4 \%)$, largely due to elastic wave scattering.

It is worth noting that 100 data points were selected to balance the need of approximating the number of data points collected from a single patient in a clinical setting and stability of curving fitting using Eqns. (4.6-4.9). We verified that the relative difference of the estimated parameters using AIC, BIC were within $2 \%$ when we varied the number of data points from 100 to 200 . In a similar note, the grid size of the computing domain 
(see Fig. 4.4) was set to $0.1 \mathrm{~mm}$. This choice was a balance of tradeoffs between the computational cost and the modeling realism. If the grid size would be further reduced the computational cost (mainly memory requirements and computational time) would increase exponentially. If a too large value of the grid size would have been chosen, the computer model may not be able to represent architectural changes at the tissue level (see Fig. 4.3).

There are limitations of the current study. First, in this preliminary study, we used a 2D tissue model along with 2D SW simulations to verify the proposed PDF, which is not limited to 2D based on the derivation (see Appendix D). In 2D SW simulations, complicated wave propagation phenomena such as mode conversion and wave splitting in heterogeneous and perhaps locally anisotropic media cannot be realistically mimicked. Of note, 2D SW simulations have been selected because computational demands of 3D SW simulations in K-Wave are prohibitively high given our computational resources. Second, as explained before in the Section Materials and Methods, the current setting of SW simulations is close to TE or Magnetic Resonance Imaging-based SWE. Acoustic radiation force-induced SWE has more complex SW wave fronts. In the future, 3D M-C simulations of acoustic radiation force-induced SWE will be repeated for further verifications. We are in the process of extending an advanced ultrasound simulation platform [84] for SWE simulations. Third, also due to the limitation of computational resources, we selected a small computing domain (an approximately $90 \mathrm{~mm} \times 22 \mathrm{~mm}$ ) containing a $20 \mathrm{~mm} \times 20$ $\mathrm{mm}$ ROI. The dimension perpendicular to the SW propagation direction is on the order of SW wavelength investigated. Because of the periodic boundary (see Appendix D), this setting is different than SW waveguides. In SW waveguides, the outward SW motion is 
often highly constrained. We also varied the size of the ROI to investigate whether the SWS is sensitive to the size of the ROI (see Figure 4). Under the same conditions, outcomes between three different domain sizes (i.e. $10 \mathrm{~mm} \times 10 \mathrm{~mm}, 20 \mathrm{~mm} \times 20 \mathrm{~mm}$ and $30 \mathrm{~mm}$ $\times 30 \mathrm{~mm}$ ) differed within $5 \%$. Some small differences are expected given the nature of MC simulations.

In this work, we describe options chosen and provide justifications for our choices. Overall, the study design is appropriate for the first feasibility study, despite limitations. Future work includes 3D SW simulations with a 3D stochastic tissue model and applications of the proposed PDF to biological data.

\subsection{Conclusion}

In summary, a PDF of the SWS distribution in multi-phase stochastic media was derived by the principle of ME. Given preliminary results presented in this study, this derived PDF can perform well for fitting SWS measurements data. Also, the parameters involved in the derived PDF can be related to the mechanical properties of the medium being imaged. This new method does seem to add value and could represent a feasible path to the study uncertainties in SWE due to tissue heterogeneity. This initial feasibility study is encouraging and we believe that the method warrants further studies. 


\section{Chapter 5. Influence of Tissue Microstructures on Shear Wave Speed Measurements in Transient Elastography ${ }^{4}$}

\subsection{D Random Two-phase Models of Fibrotic Liver Tissues}

Because liver fibrosis without steatosis results in depositions of stiff collagenous scar tissues embedded in the normal liver parenchyma [118], fibrotic liver tissues can be simplified as a two-phase medium consisting of two components - normal background and collagenous depositions as a first approximation. Without the loss of generality, we regarded the connected collagenous fibers as the inclusions with an area fraction of $\alpha$. However, these collagen depositions could be arbitrarily distributed.

Three two-phase models were investigated to show how spatial characteristics of these inclusions can influence (group) SWS measurements in TE. As shown in Fig. 5.1, the first model (Model 1) was designated to mimic histological characteristics of fibrosis under the METAVIR classification [105]. In the early stages of liver fibrosis (i.e. METAVIR scores F1 and F2; CPA = 10\%), liver injuries occur and collagen is deposited around portal tracts. Those collagen depositions look like isolated islands (See the top left plot in Fig. 5.1). As the liver fibrosis progresses (i.e. METAVIR scores F3 or F4; CPA = $30 \%$ ), fibrous expansions of most portal areas cause portal-to-portal bridging or portal-tocentral bridging [107], as shown in the top right plot in Fig. 5.1.

As also shown in Fig. 5.1, the second and third models are mathematical models. Particularly, Model 2 is a statistics-based model [119]. A spatial Gaussian random field

\footnotetext{
${ }^{4}$ The material contained was submitted to Ultrasound Imaging, 2017.
} 
was first generated for local tissue shear moduli. Then, a cut-off threshold of the tissue shear modulus values was used to convert the spatial Gaussian random field to a binary (two-phase) random medium. The reconstruction process was done using a nonlinear transformation [119] that ensures respective correlation lengths of those initial spatial Gaussian random fields were retained in all resulting random (two-phase) medium. In Model 3, rectangular inclusions with a pre-determined size were added to the background until the needed area fraction ratio $\alpha$ would be reached.

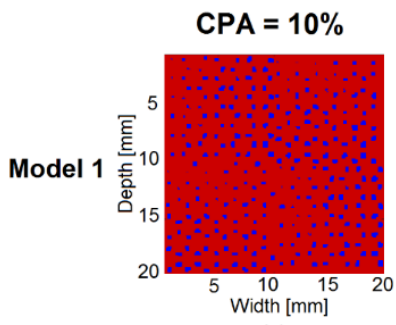

(a)

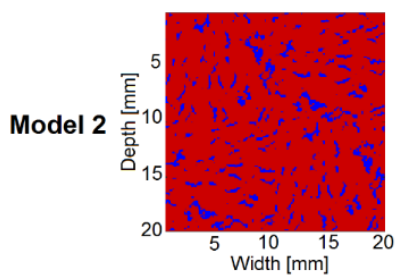

(c)

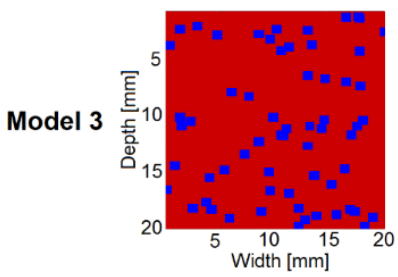

(e)

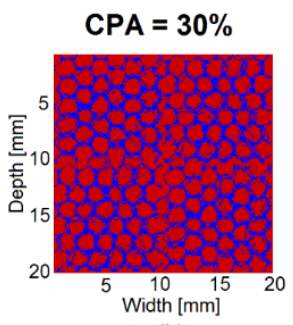

(b)

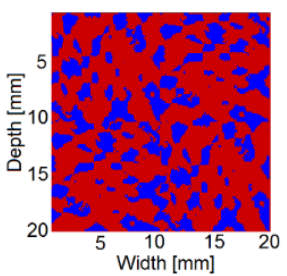

(d)

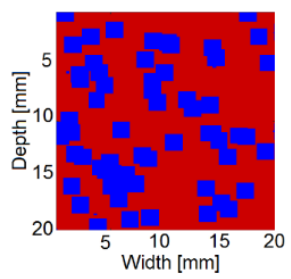

(f)

Figure 5.1. Realizations of random two-phase fibrotic liver tissues at the different collagen proportionate area (CPA): Model 1 (first row), Model 2 (second row) and Model 3 (third row). The first and second columns represent two different CPAs: 10\% and $30 \%$, respectively. In all plots, blue and red colors represent collagenous (connective) tissues and normal liver tissues, respectively. 


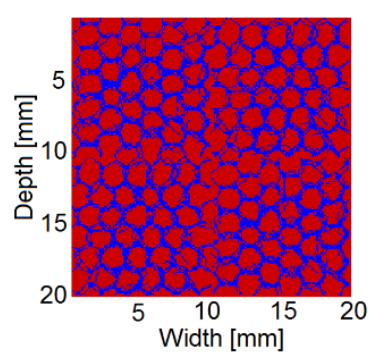

(a)

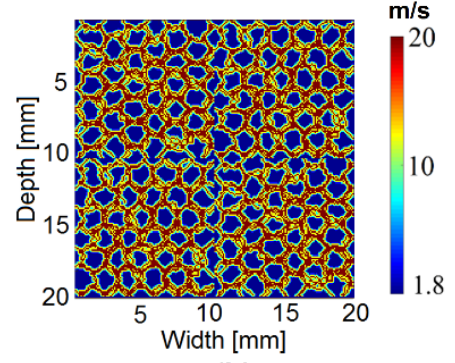

(b)

Figure 5.2. An illustration of the process of simulated "cross-linking": (a) the original model from Figure 1 and (b) a micro-mechanical model after applying the Gaussian smoothing. In (b), the color bar represents SWS in $\mathrm{m} / \mathrm{s}$.

In order to match SWS values of simulated liver tissues with METAVIR scores [105] for early and late stages of liver fibrosis as reported in the clinical literature [120122], an image processing technique mimicking elevated cross-linking [123] was used. Basically, after applying the smoothing kernel, the virtual tissues adjacent to collagenous depositions were hardened as shown in Fig. 5.2b. In the Gaussian smoothing model, we reduced two parameters (i.e. kernel radius and standard deviation of Gaussian distribution) to one by setting the (spatial) kernel size to $2 \sigma$, where $\sigma$ is the standard deviation of the Gaussian smoothing kernel. $2 \sigma$ approximately covers $95 \%$ of the data arrange. Thus, the only parameter for the Gaussian smoothing kernel was its (spatial) kernel size. The kernel sizes were set to be $0.2 \mathrm{~mm}$ for both CPAs of $10 \%$ and $30 \%$.

\subsection{Numerical Simulations}

As shown in Fig. 5.3, elastic shear wave propagation was simulated by K-Wave [124], which is a spectral finite difference solver of the elastic wave equation. The overall dimensions of computing domain are $90 \mathrm{~mm}$ (along the shear wave propagation direction; 
see the arrow in Fig. 5.3) $\times 22 \mathrm{~mm}$. There are a $20 \mathrm{~mm} \times 20 \mathrm{~mm}$ central rectilinear region of interest (ROI) containing the two-phase models of fibrotic liver tissues after the simulated "cross-linking" process; the rest of the computing domain is uniform.

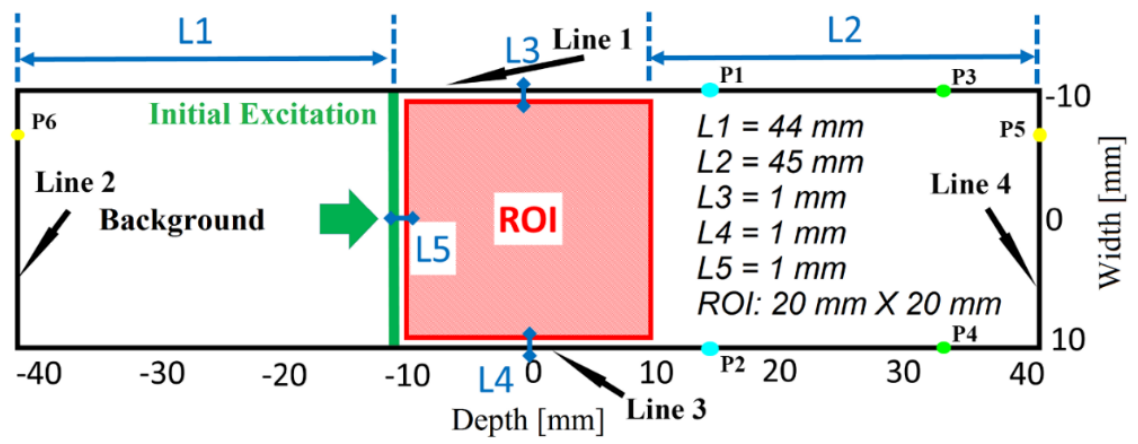

Figure 5.3. An illustration of computing domain used for K-Wave simulation. A $(20 \mathrm{~mm}$ $\times 20 \mathrm{~mm}$ ) region of interest (ROI) containing the two-phase models of fibrotic liver tissues was embedded into a larger $(90 \mathrm{~mm} \times 22 \mathrm{~mm})$ uniform background. The green vertical line indicates the location of initial mechanical vibration, while the green arrow represents the shear wave propagation direction. The initial excitations are vertical to the wave propagation direction.

One transient cycle of the plane shear wave excitations at one of the following nine frequencies: $50 \mathrm{~Hz}-400 \mathrm{~Hz}$ with an increment of $50 \mathrm{~Hz}$, was applied to the left edge of the ROI (see the green line in Fig. 5.3) to initiate the shear wave propagation. One cycle of transient plane shear wave excitation including the frequency range $(50-400 \mathrm{~Hz})$ was selected to mimic TE [31]. Periodic (also known as mirroring) boundary conditions have been applied to four edges (i.e. Lines 1-4 in Fig. 5.3). The mirroring boundary condition will ensure that time-resolved velocity and stress between any pair of corresponding sites around edges of the computing domain (e.g. P1 and P2 [Lines 1 and 3], P3 and P4 [Lines 1 and 3], P5 and P6 [Lines 2 and 4]). All computing grid points located at four edges were paired and the mirroring boundary was enforced among all identified pairs. Since the 
mirroring boundary condition doesn't constrain motion along any edges of the model (see Fig. 5.3), our computing domain should not be considered as a model of waveguide. A more formal description of the simulation setting can be found in a prior publication (i.e. Appendix B of [125]).

The required mechanical properties used in K-WAVE simulations were adopted from the literature $[90,109,110,126]$ and are summarized in Table 5.1. Normal liver shear modulus was set to be around $3.24 \mathrm{kPa}$ (equivalent to the SWS of $1.8 \mathrm{~m} / \mathrm{s}$ ) [109]. The shear modulus value of fibrotic liver tissues was set to $400 \mathrm{kPa}$ (equivalent to SWS of $20 \mathrm{~m} / \mathrm{s}$ ) [110]. The grid spacing within the entire computing domain was uniform and the pixel/grid size was $0.1-\mathrm{mm} \times 0.1-\mathrm{mm}$. This selected grid size resulted in at least 20 points per wavelength and should adequately resolve the shear wave propagation. Mesh sensitivity tests were performed to verify that results were not sensitive to the chosen grid size. Compression and SW velocity parameters (i.e. compressional sound speed, shear wave speed, and mass density) were assigned to every pixel in the rectilinear computing grid.

Table 5.1 Mechanical properties of the two-phase models of fibrotic liver tissues used in the K-WAVE simulations. SWS and SOS denote shear wave speed and speed of sound, respectively.

\begin{tabular}{ccc}
\hline & $\begin{array}{c}\text { Normal Liver } \\
\text { Tissue }\end{array}$ & Fibrotic Tissue \\
\hline \hline SWS $(\mathrm{m} / \mathrm{s})$ & 1.8 & 20 \\
SOS $(\mathrm{m} / \mathrm{s})$ & 1540 & 1800 \\
Mass Density $\left(\mathrm{kg} / \mathrm{m}^{3}\right)$ & 1000 & 1100 \\
\hline
\end{tabular}

\subsection{Data Analysis}


Once the simulated SW velocity data were obtained, a direction filter [103] was applied to the simulated wave data to remove wave interferences. Then, a lateral time-topeak (TTP) algorithm [44] was adopted to estimate SWS along the shear wave propagation direction (see the arrow in Fig. 5.3). More specially, we fitted identified local peaks (yaxis) with respect to their lateral locations ( $\mathrm{x}$-axis) to a line and the slope became the propagating SWS. The lateral kernel length was $1.2 \mathrm{~mm}$. Key simulation details can be found in Table 5.2.

Table 5.2 Simulation details of the K-Wave simulation. The correlation length was quantified using two-point correlation function [112]. The two-point correlation length was used to characterize the approximate size of inclusions on an average sense.

\begin{tabular}{cccccc}
\hline CPA & $\begin{array}{c}\text { Excitation } \\
\text { Frequency }(\mathrm{Hz})\end{array}$ & $\begin{array}{c}\text { Number of } \\
\text { Realizations } \\
\text { for Each } \\
\text { Excitation } \\
\text { Frequency }\end{array}$ & $\begin{array}{c}\text { Correlation } \\
\text { Length }\end{array}$ & $\begin{array}{c}\text { Number of } \\
\text { SWS } \\
\text { Measurements } \\
\text { for Each } \\
\text { Excitation } \\
\text { Frequency }\end{array}$ \\
\hline \hline Model & $10 \%$ & $50,100,150,200$ & 5 & $0.6 \mathrm{~mm}$ & 11,000 \\
$\mathbf{1}$ & $30 \%$ & $50,100,150,200$ & 5 & $1.5 \mathrm{~mm}$ & 11,000 \\
\hline Model & $10 \%$ & $50,100,150,200$ & 5 & $0.6 \mathrm{~mm}$ & 11,000 \\
$\mathbf{2}$ & $30 \%$ & $50,100,150,200$ & 5 & $1.5 \mathrm{~mm}$ & 11,000 \\
\hline Model & $10 \%$ & $50,100,150,200$ & 5 & $0.6 \mathrm{~mm}$ & 11,000 \\
$\mathbf{3}$ & $30 \%$ & $50,100,150,200$ & 5 & $1.5 \mathrm{~mm}$ & 11,000 \\
\hline \hline
\end{tabular}

Instead of analyses of all 11,000 SWS data together (see Table 5.2), we randomly selected 100 data points from all 11,000 data points to mimic "clinical" SWS data. This process was repeated 100 times to generate 100 realizations of simulated "clinical" SWS data for each "parameter setting" [125]. One "parameter setting" is defined as a unique 
combination of the model (Models 1-3), CPA (10\% and 30\%) and excitation frequency (50 $-400 \mathrm{~Hz})$. Then, the mean and standard derivation of the SWS distribution were estimated by fitting data in each "parameter setting" into a probability density function (PDF) derived from the Maximum Entropy Theorem [125],

$$
p^{M E T}\left(V_{S}\right)=\frac{1}{\sqrt{2 \pi G_{D}}} e^{\frac{-\left(V_{S}^{2} \rho-G_{E}\right)^{2}}{2 G_{D}}} 2 \rho V_{S}
$$

where $G_{E}$ and $G_{D}$ are mean and variance of the effective shear modulus $G$, respectively. In Eqn. (1), $\rho$ is the mass density.

To estimate frequency-dependent SWS dispersion, the estimated group SWS data were first plotted as a function of frequencies between $50-400 \mathrm{~Hz}$. Then, the dispersion slope $\left(d_{V_{s}} / d_{f}\right)$ was estimated by a linear regression.

\subsection{Simulation Results}

Means and standard deviations of the underlying shear modulus values fitted into Eqn. (1) are shown in Fig. 5.4-5.6 as a function of frequency for Models 1-3, respectively. In Fig. 5.4-5.6, we found that the group SWS estimates were dependent on frequencies regardless of the underlying microstructure model. Furthermore, there was increasing SWS dispersion with the increase of CPAs. Taking Model 1 as an example, the dispersion slope $d_{V_{S}} / d_{f}$ increased from $0.81 \mathrm{~m} / \mathrm{s} / \mathrm{kHz}$ to $2.19 \mathrm{~m} / \mathrm{s} / \mathrm{kHz}$ when increasing CPA from $10 \%$ to $30 \%$. It is also noticed that different underlying microstructure distributions could result in varying degree of shear wave dispersion. For instance, the largest differences between estimated dispersion slopes $\left(d_{V_{S}} / d_{f}\right)$ were between Model 1 and Model 3. Quantitatively, those differences were $31 \%$ and $63 \%$ for $10 \% \mathrm{CPA}$ and $30 \% \mathrm{CPA}$, respectively. 
Quantitative comparisons of these estimated dispersion slopes are summarized in Table 5.3 below.

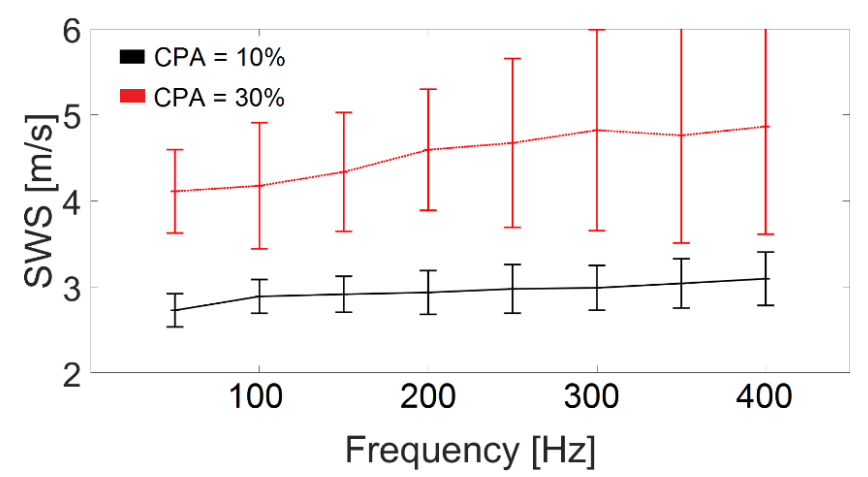

Figure 5.4. Plots showing mean SWS $(\mathrm{m} / \mathrm{s})$ as a function of the excitation frequencies in Model 1. Two different CPAs: (a) 10\% and (b) 30\% were used. Error bars represent one standard deviation $(\mathrm{m} / \mathrm{s})$.

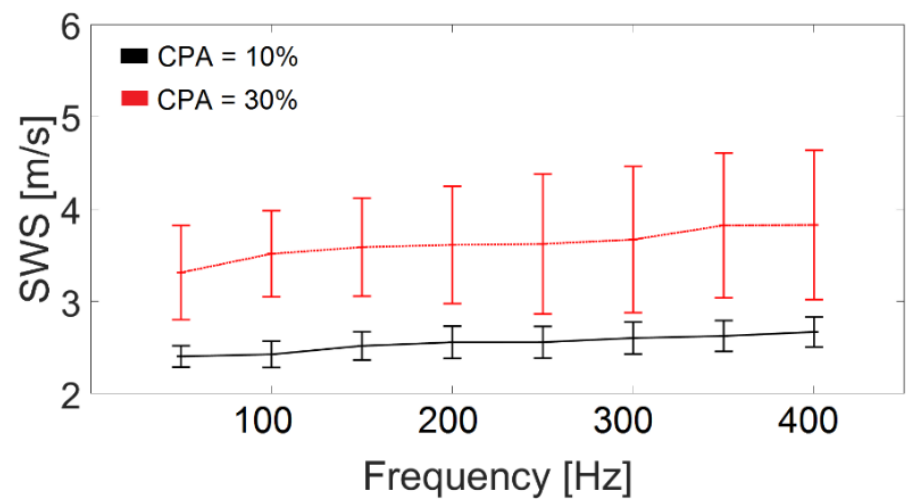

Figure 5.5. Plots showing mean SWS $(\mathrm{m} / \mathrm{s})$ as a function of the excitation frequencies in Model 2. Two different CPAs: (a) 10\% and (b) 30\% were used. Error bars represent one standard deviation $(\mathrm{m} / \mathrm{s})$. 


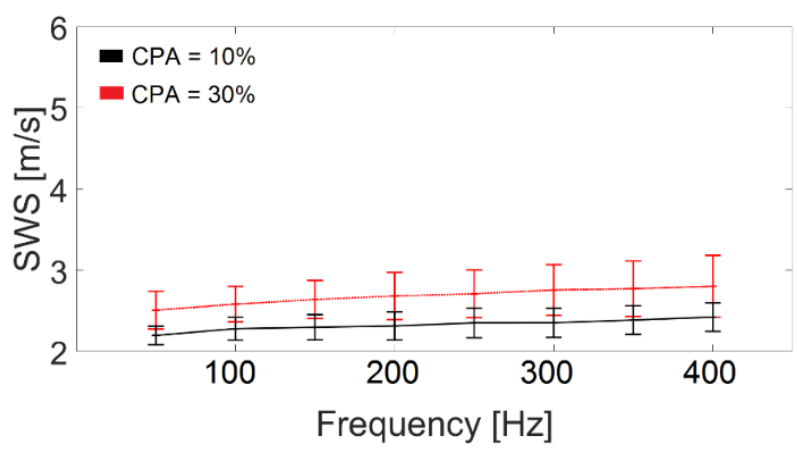

Figure 5.6. Plots showing mean SWS $(\mathrm{m} / \mathrm{s})$ as a function of the excitation frequencies in Model 3. Two different CPAs: (a) 10\% and (b) 30\% were used. Error bars represent one standard deviation $(\mathrm{m} / \mathrm{s})$.

Table 5.3 A summary of fitted $d_{V_{s}} / d_{f}$ from three tissue models. $10 \%$ and $30 \%$ of CPAs were considered in the K-WAVE simulations. The $R^{2}$ is a metric of measuring goodness of fit for $d_{V_{S}} / d_{f}$.

\begin{tabular}{lccccc}
\hline & \multicolumn{2}{c}{$\mathbf{1 0 \%}$} & \multicolumn{2}{c}{$\mathbf{3 0 \%}$} \\
& $\begin{array}{c}d_{V_{s}} / d_{f} \\
(\mathrm{~m} / \mathrm{s} / \mathrm{kHz})\end{array}$ & $R^{2}$ & $\begin{array}{c}d_{V_{s}} / d_{f} \\
(\mathrm{~m} / \mathrm{s} / \mathrm{kHz})\end{array}$ & $R^{2}$ \\
\hline \hline Model 1 & 0.81 & 0.88 & 2.19 & 0.91 \\
\hline Model 2 & 0.72 & 0.95 & 1.24 & 0.87 \\
\hline Model 3 & 0.56 & 0.94 & 0.81 & 0.93 \\
\hline \hline
\end{tabular}




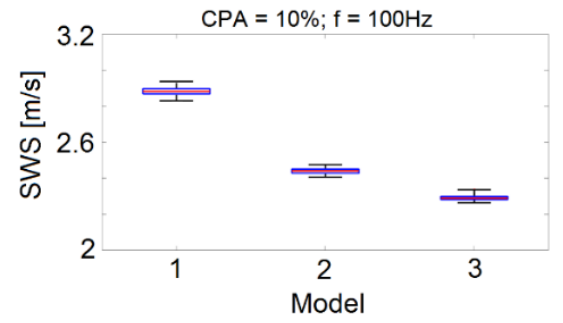

(a)

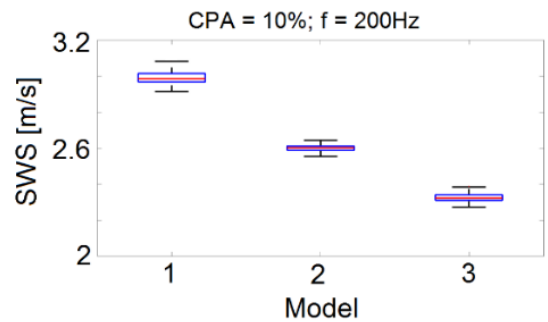

(c)

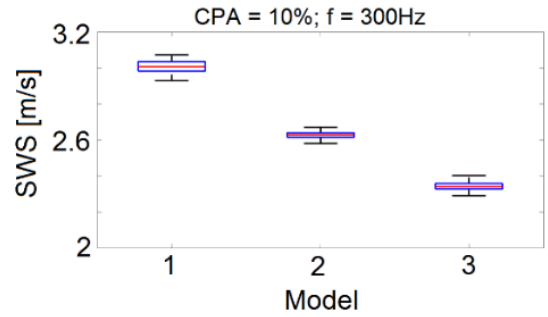

(e)

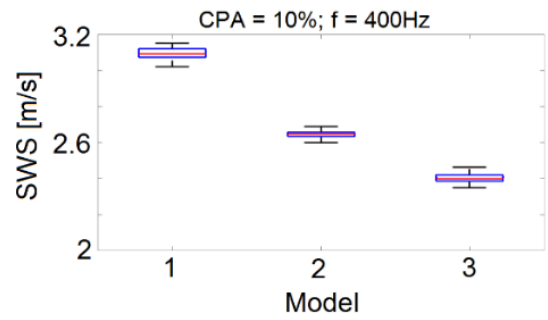

(g)

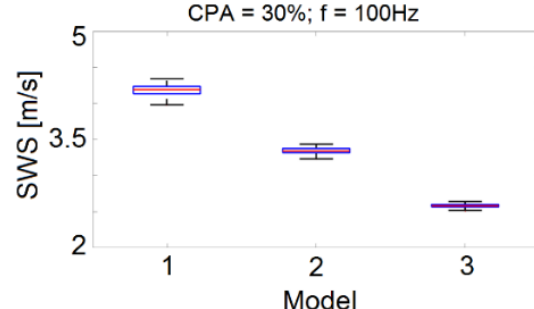

(b)

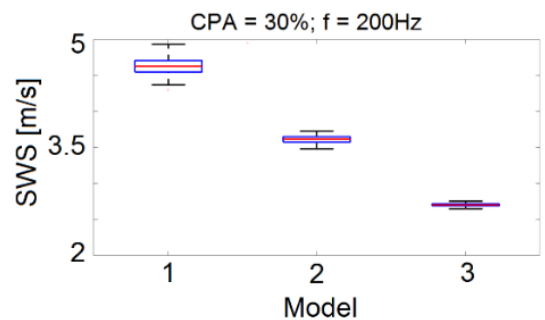

(d)

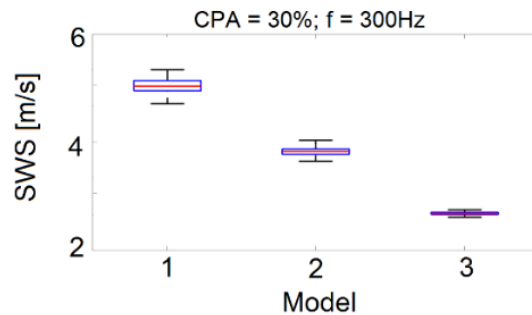

(f)

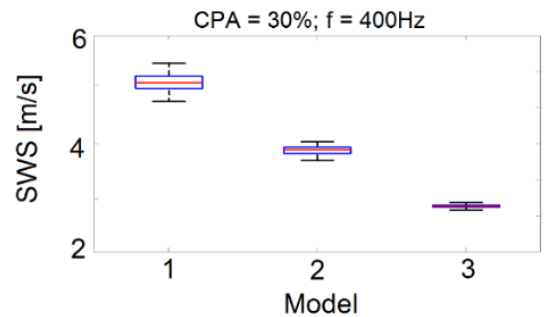

(h)

Figure 5.7 Clustered box plots obtained from mean group SWS values for three tissue models. The top and bottom of the boxes indicate 75 and 25 percentiles, respectively. The line through the middle of each box represents the median. The error bars show the minimum and maximum values.

Recall that there were 48 combinations given different tissue models (Models 1-3), excitation frequencies $(50 \mathrm{~Hz}-400 \mathrm{~Hz}$, increment $50 \mathrm{~Hz})$ and CPAs $(10 \%$ and $30 \%)$. In each 
combination, there were 100 sets of simulated "clinical" group SWS data; each set contained 100 simulated group SWS measurements. In each set, 100 simulated group SWS measurements were fitted to the derived PDF (see Eqn. 1) to obtain the mean group SWS for the data set. Box plots of the mean values of the group SWS (y-axis) are shown in Fig. 5.7a-h for excitation frequencies $(100,200,300$ and $400 \mathrm{~Hz})$. It is easy to see that, under the same excitation frequency and the CPA, the mean group SWS values were different among Models 1-3. Overall, based on the Wilcoxon rank sum tests, these differences shown in Fig. 5.7a-h were all statistically significant $(\mathrm{p}<0.001)$.

\subsection{Discussion}

Results in Figs. 5.4-5.6 also indicated that different mean/median values of shear moduli were obtained when three different microstructure fibrotic liver models were used to perform the shear wave simulations. Such differences were significant. Taking the $30 \%$ CPA and the excitation frequency of $200 \mathrm{~Hz}$ as an example, the median values of the mean group SWS values were around $4.6 \mathrm{~m} / \mathrm{s}, 3.5 \mathrm{~m} / \mathrm{s}$ and $2.6 \mathrm{~m} / \mathrm{s}$ for Model 1, Model 2 and Model 3 , respectively. The relative difference was as high as $43 \%$ between the Model 1 and Model 3. In the clinical literature [120-122], group SWS values were on average about $3 \mathrm{~m} / \mathrm{s}$ and $4.5 \mathrm{~m} / \mathrm{s}$ for METAVIR classifications F2 and F4 tissues, respectively. Consequently, results obtained from Model 1 (see Fig. 5.7) were most consistent with those clinical data [120-122]. However, if the emulated "cross-linking" effect had not been implemented, the group SWS values of Model 1 (Fig. 5.8) would have remained approximately $3 \mathrm{~m} / \mathrm{s}$ for a $30 \%$ CPA.

As also shown in Fig. 5.8, the group SWS increased in Model 1 when the shear 
moduli of those collagen depositions had been varied between $50-400 \mathrm{kPa}$. The combination of emulated "cross-linking" and setting collagen depositions to $400 \mathrm{kPa}$ made the group SWS elevated to approximately $4.2 \mathrm{~m} / \mathrm{s}$ for the CPA of $30 \%$. This number seems consistent with those reported in the clinical literature for late stages of liver fibrosis [120122].

It is also worth noting that, even with the emulated "cross-linking" effect, Models 2 and 3 yielded significantly lower group SWS values (see Fig. 5.7) as compared to data reported in the clinical literature. Intuitively, the histology-based Model 1 containing collagen depositions around liver septa and therefore, the emulated "cross-linking" by a Gaussian Smoothing kernel was most effective. In the current study, the emulated "crosslinking" was assumed to be isotropic as a first approximation, given a paucity of experimental data on this topic.

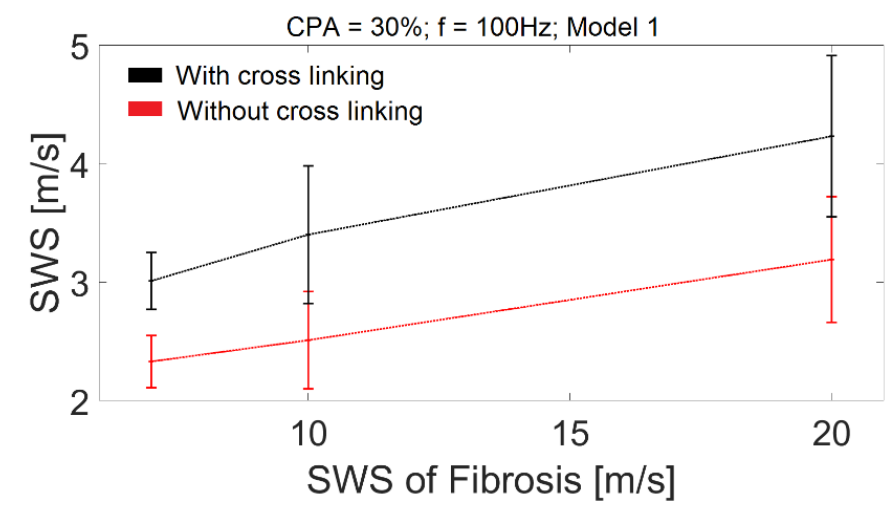

Figure 5.8. A plot showing changes in estimated group SWS value with respect to the stiffness changes of collagenous dispositions. Error bars denote a standard deviation.

Interestingly, the work by Nightingale et al. [127] showed that the phase velocity slope with respect to the excitation frequency increases from $2.92 \mathrm{~m} / \mathrm{s} / \mathrm{kHz}$ for one 
(METAVIR classifications) F1 human case to $4.05-\mathrm{m} / \mathrm{s} / \mathrm{kHz}$ for an F3 (METAVIR classifications) human case. Our results shown in Table 5.3 suggested that, in Model 1, the group SWS change with respect to the excitation frequency was approximately 2.19$\mathrm{m} / \mathrm{s} / \mathrm{kHz}$ in simulated data when the CPA was $30 \%$. The CPAs of $10 \%$ and $30 \%$ correlate to early (METAVIR classifications F1-F2) and late (METAVIR classifications F3-F4) stages of liver fibrosis, respectively [108]. Because we used a single frequency excitation, we were unable to calculate the dispersion slopes following the same method used by Nightingale et al. [127]. Nevertheless, the shear wave dispersion from the elastic (shear) wave scattering appeared to be significant and thus should be further investigated in tissuemimicking materials and biological tissues. Perhaps, a notable contribution offered by our study is to increase the awareness of elastic shear wave scattering and its potential impact on the frequency-dependent shear wave dispersion. In this study, elastic wave scattering is within the Rayleigh Regime.

Current experimental and clinical studies [127-130] implicitly assumed that the source of frequency-dependent shear wave dispersion stemmed from the viscosity by the adoption of the well-known Vigot model. Given the nature of biological tissues, the viscosity is likely a source of wave dispersion and this study should not be interpreted as a challenge to previous studies [127-130]. Instead, we are interested in understanding whether elastic scattering in heterogeneous lossless media could be a source of frequencydependent shear wave dispersion.

To obtain simulated SWS distributions, the linear regression kernel length was 1.2 $\mathrm{mm}$. To verify the sensitivity of the kernel length, our results were reprocessed using a 
smaller kernel length of $0.4 \mathrm{~mm}$. We found that the means of SWS remained to be the same but the variances of SWS were increased by approximately $5-8 \%$.

There are limitations of the current study. First, we used a 2D tissue model along with 2D SW simulations. It is important to note that, in 2D SW simulations, complicated wave propagation phenomena such as mode conversion and wave splitting in heterogeneous and perhaps locally anisotropic media cannot be realistically mimicked. Of note, 2D SW simulations have been selected because computational demands of 3D SW simulations in K-Wave are prohibitively high given our computational resources. Second, as explained before in Section 2, the current setting of SW simulations is close to TE or Magnetic Resonance Elastography. Acoustic radiation force-induced SWE has more complex SW wave fronts and will induce (tissue) excitations with broader frequency components. Work is needed to extend observations reported in this study to acoustic radiation force-induced SWE [1]. Third, we were unable to combine both elastic scattering and viscosity-related dispersion together in this study because, in the open-source KWAVE, frequency-dependent SWS has not been implemented for the individual material phases in a composite. The frequency-dependent absorption in K-WAVE was tested though data were not reported to keep this study concise. Our initial testing showed that attenuation of shear wave amplitude had neglectable impacts on our simulation results if the (shear) wavefronts were trackable.

In the future work, we will further investigate combined effects of elastic scattering and viscosity for acoustic radiation force-induced SWE in an open-source ultrasound elastography simulation platform $[84,131]$. 


\subsection{Conclusions}

In summary, a PDF of the SWS distribution in multi-phase stochastic media was derived by the principle of ME. Given preliminary results presented in this chapter, this derived PDF can perform well for fitting SWS measurements data. Also, the parameters involved in the derived PDF can be related to the mechanical properties of the medium being imaged. This new method does seem to add value and could represent a feasible path to the study uncertainties in SWE due to tissue heterogeneity. This initial feasibility study is encouraging and we believe that the method warrants further studies. Also, we found underlying microstructures affected the estimated group SWS as much as $44 \%$. Intuitively, the histology-based Model 1 containing collagen depositions around liver septa and therefore, the emulated "cross-linking" by a Gaussian Smoothing kernel was most effective. Consequently, results obtained from Model 1 were most consistent with those clinical data. It is important to note that the frequency-dependent shear wave dispersion investigated in this chapter is different as compared to the data available in the literature. In this chapter, we focused on elastic scattering as a potential source of shear wave dispersion. It is easy to see that the presence of inclusions led to some distortions of wave fronts, impacting measurements in the nominal group SWS. This new finding may motivate further studies examining how elastic scattering may contribute to frequencydependent shear wave dispersion in biological tissue. 


\section{Chapter 6. A Two-dimensional (2D) Systems Biology- based Micromechanical Liver Tissue Model: A Simulation Study with Implications for Ultrasound Elastography of Liver Fibrosis 5}

Continuum tissue models are often used to simulate or analyze mechanical properties of tissues being imaged by ultrasound elastography (UE). However, those continuum models may not be biologically realistic. Chapter 6 here was to establish the feasibility of using systems biology to construct biologically relevant tissue models linking tissue structure, composition and architecture to the ultrasound measurements directly. In this feasibility, the first application was designated to model fibrotic liver tissues.

As shown in Fig. 6.1, interactions between the SB based virtual liver tissue model and simulations of compression and shear wave propagation involve several major steps. All steps, along with the use of two open-source simulation platforms, namely a wave simulator (K-WAVE [124]) and an agent-based knowledge simulator (SPARK [132]), are briefly presented below.

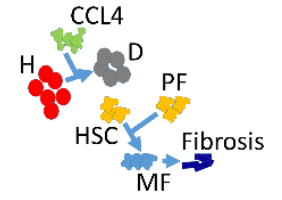
SB Simulation

(a)

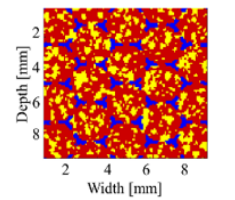

Virtual Liver Tissue

(b)

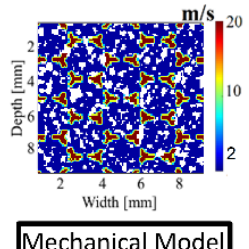

(c)

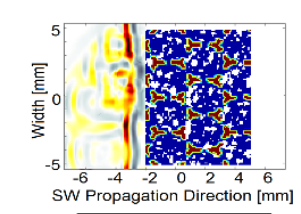

Wave Simulation

(d)

Figure 6.1. Schematic illustrations of the workflow: (a) Systems biology [SB] simulation, (b) virtual liver tissue generated from SB simulation, (c) mechanical model of the virtual liver tissue and (d) wave simulations by K-WAVE. The wave propagates from left to right in (d). In (a), different color represents different agents whose acronyms

\footnotetext{
${ }^{5}$ The material contained in this chapter will be submitted to Ultrasound in Medicine and Biology, 2017.
} 
can be in Figure 2, while red, blue and yellow represent normal liver tissue, fibrosis, and fat, respectively. In (c), the color bar denotes SWS in $\mathrm{m} / \mathrm{s}$.

\subsection{Virtual Liver Tissue Model}

We treated the fibrotic liver tissue as a three-phase random medium where the normal liver parenchyma and the deposits of excessive connective collagen tissues and lipids are the background and inclusions, respectively. Architectural features of those three components were simulated using SB. To do so, we expanded and modified an existing agent-based SB model of liver fibrosis originally developed by others [60] in SPARK. Signaling pathways for liver fibrosis, liver steatosis, and interactions between the liver fibrosis and steatosis are shown in Fig. 6.2. We first describe simulations of liver fibrosis and steatosis as two independent processes and then outline their interactions on knowledge available [133]. More details can be found in $\underline{\text { Appendix E. }}$.

Toxicity based liver fibrosis, due to the injection of carbon tetrachloride $\left(\mathrm{CCL}_{4}\right)$, was induced through four parallel schemes (Fig. 6.2). In the first scheme, liver injuries induced by $\mathrm{CCL}_{4}$ transform normal hepatocytes into dead agents. $\underline{\text { In the second scheme, }}$ once Kupffer cells sense dead hepatocytes, they immediately phagocytize and activate the production of TNF- $\alpha$ (a canonical pro-inflammatory cytokine) and TGF- $\beta 1$ (a canonical anti-inflammatory cytokine). In the third scheme, assuming inadequate clearance of dead hepatocytes occurs, damage-associated molecular pattern (DAMP) molecules such as HMGB1 starts to accumulate. Certainly, the presence of HMGB1 would subsequently recruit and convert more monocytes to active Kupffer cells. In the fourth scheme, agents such as active portal fibroblasts and HSCs transform to myofibroblast agents when they 
detect TNF- $\alpha$. Similarly, when the system detects TGF- $\beta 1$, myofibroblast agents start to proliferate and deposit collagen to existing ECM structure. Of note, in all four abovementioned schemes, interactions among them were probabilistic.

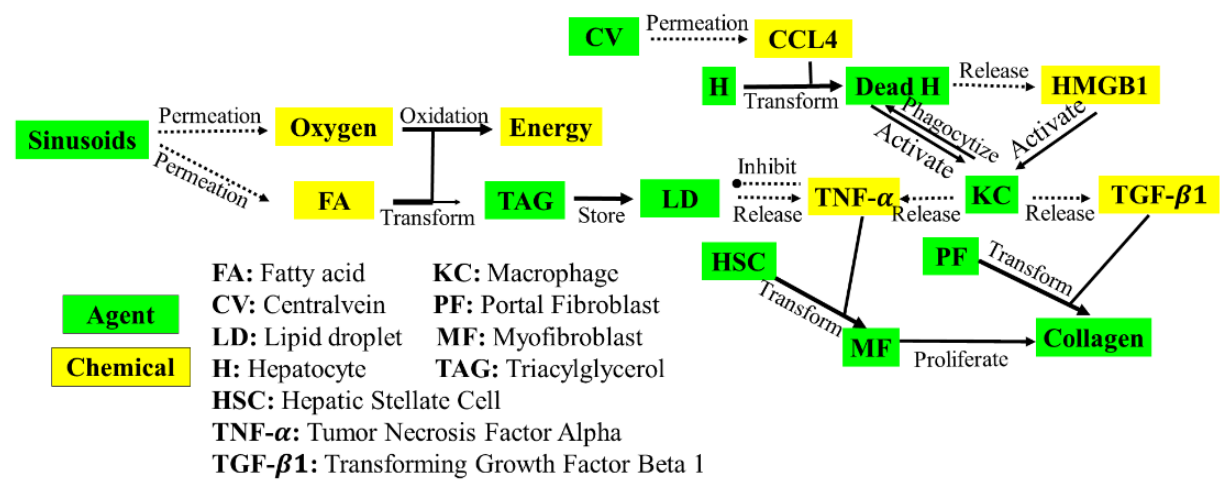

Figure 5.2. A diagram showing signaling pathways related to liver fibrosis and steatosis induced by $\mathrm{CCL}_{4}$ insults induced liver fibrosis and lipid accumulation, respectively.

Now referring to the simulation of liver steatosis, we adopted a theoretical model developed by Schleicher et al. [134]. More specifically, the active scheme (hereafter referred as to the fifth scheme) [134] is that the storage of triacylglycerol (TAG) within hepatocytes leads to cell enlargement, thereby reducing the sinusoids radius and impairing hepatic microcirculation. Because of the compromised microcirculation, oxygen supplies will be reduced and, consequently, lipid oxidation will be further impaired. Eventually, lipid accumulation will occur in the liver (Fig. 6.2). Currently, the dynamic interactions between the liver steatosis and fibrosis are complex and not fully understood. Based on work by Diehl et al. [135], we assumed that the presence of lipid droplets up-regulate TNF$\alpha$. As a multi-functional cytokine, TNF- $\alpha$ is actively involving in energy metabolism, especially lipid homeostasis. This interaction is also illustrated in Fig. 6.2. 


\subsection{Virtual Tissue Model for Simulations of Ultrasound Compressional/Shear Waves}

\subsubsection{Creation of Computing Grid}

The SB-based simulations resulted in random three-phase media (Fig. 6.1b). Since K-WAVE [124] solves wave equations based on a K-space finite difference scheme, a "homogenization/averaging" scheme (Fig. 6.3a-b) [136] was used to convert microscopic compositions of tissues (e.g. cellular components) to represent a discrete finite difference grid (Fig. 6.3b) whose grid size ranged from 50 to 100 micrometers. The 100-micrometer grid spacing (>100 grid points per wavelength) was used for simulations of ultrasound transient elastography (TE) [31] while the compression wave simulations at $2 \mathrm{MHz}$ were performed using a grid size of 50-micrometers (i.e. approximately 15 grid points per wavelength).

To mimic an assumed cross-linking process [123] in fibrotic liver tissues, a Gaussian smoothing kernel was applied onto the initial discretized computing grid (Fig. 6.3b). After applying the smoothing kernel, the virtual tissues were hardened as shown in Fig. 6.3c. In the Gaussian smoothing model, we reduced two parameters (i.e. kernel radius and standard deviation of Gaussian distribution) to one by setting the (spatial) kernel size to $2 \sigma$, where $\sigma$ is the standard deviation of the Gaussian smoothing kernel. $2 \sigma$ approximately covers $95 \%$ of the data arrange. Thus, the only parameter for the Gaussian smoothing kernel was its (spatial) kernel size. It is also important to note that, in the three-

phase model, we stipulated that the Gaussian smoothing kernel does not interfere with the 
presence of fatty tissues because histological evidences suggested that fibrosis typically spans around adipocytes [137].

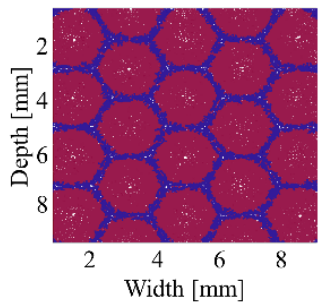

Virtual Liver Tissue

(a)

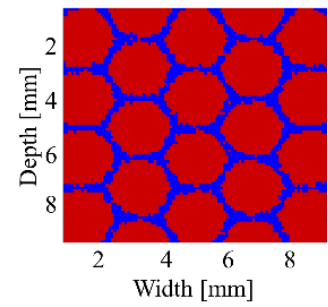

Homogenization

(b)

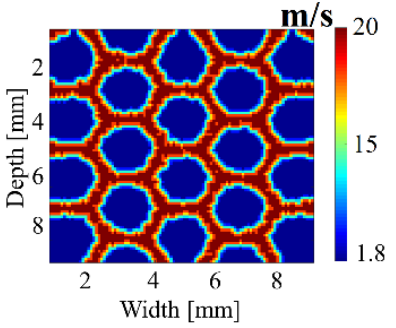

Gaussian Smoothing

(c)

Figure 6.3. An illustration of the process of "Homogenization": (a) the original model generated by SPARK, (b) a converted model by "averaging" from (a) and (c) a micromechanical model after applying the Gaussian smoothing. In (c), the color bar represents SWS in $\mathrm{m} / \mathrm{s}$.

\subsubsection{Virtual Wave Simulations}

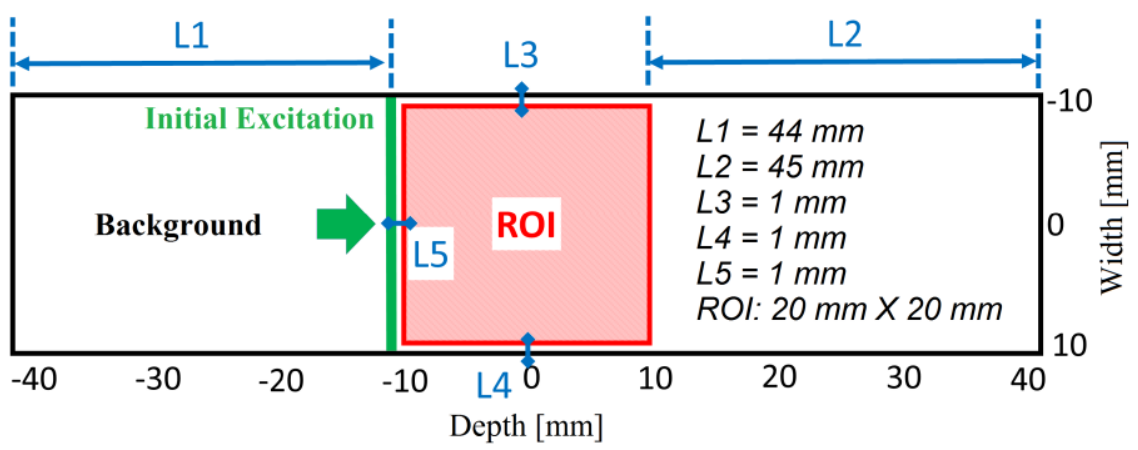

Figure 6.4. An illustration of computing domain used for all K-Wave simulations. A (20 $\mathrm{mm} \times 20 \mathrm{~mm}$ ) region of interest (ROI) containing simulated virtual liver tissues was embedded into a larger $(90 \mathrm{~mm} \times 22 \mathrm{~mm})$ uniform background. The green vertical line indicates the location of initial mechanical vibration, while the green arrow represents the wave propagation direction. For shear and compression wave propagations, the initial excitations are vertical and parallel to the wave propagation direction, respectively. 
A uniform $90 \mathrm{~mm}$ (along the wave propagation direction) $\times 22 \mathrm{~mm}$ computing domain was used to generate wave speed data, as shown in Fig. 6.4. A $20 \mathrm{~mm} \times 20 \mathrm{~mm}$ central rectilinear region of interest (ROI) containing the proposed virtual liver tissues (Fig. 6.3c) was embedded into the computing domain. The wave propagation was performed by K-WAVE.

For each model, one transient cycle of plane shear or compressional wave excitation at $100 \mathrm{~Hz}$ or $2 \mathrm{MHz}$, was applied to a set of locations close to the left edge of the ROI (see the green line in Fig. 6.4) to initiate the shear/compressional wave propagation. Both frequencies are consistent with those used in a clinical setting $[1,138]$. Particularly, one cycle of transient plane shear wave excitation is close to TE [31]. Periodic boundary conditions have been appropriately configured to avoid reflection on the boundaries. Details of the simulation setting can be found in our previous paper [139]. Simulation parameters (Table 6.1) were adopted from the literature [90, 109, 110, 126].

Table 6.1 A list of properties of the virtual liver tissues used in the K-WAVE simulations

\begin{tabular}{cccc}
\hline \hline & $\begin{array}{c}\text { Normal Liver } \\
\text { Tissue }\end{array}$ & Fibrotic Tissue & Fatty Lipid \\
\hline SWS $(\mathrm{m} / \mathrm{s})$ & 1.8 & 20 & 1.3 \\
SOS $(\mathrm{m} / \mathrm{s})$ & 1540 & 1800 & 1450 \\
Mass Density $\left(\mathrm{kg} / \mathrm{m}^{3}\right)$ & 1000 & 1100 & 1000 \\
\hline \hline
\end{tabular}

As shown in Table 6.1, normal liver shear modulus was set to be around $3.24 \mathrm{kPa}$ (equivalent to the SWS of $1.8 \mathrm{~m} / \mathrm{s}$ ) [109]. The shear modulus of the fatty tissues in Table 6.1 was similar to those of abdominal fat [126]. The shear modulus value of fibrotic liver tissues was elevated to be around $400 \mathrm{kPa}$ (equivalent to SWS of $20 \mathrm{~m} / \mathrm{s}$ ) [110]. Because, 
to our knowledge, there is no mechanical testing data available for fibrotic (collagenous) liver tissues at the spatial scale of 50-100 micrometers, the micro-packet of fibrotic tissues was chosen to be close to the shear modulus values of arterial tissues.

\subsubsection{Compositions of Virtual Fibrotic Livers}

The METAVIR scores clinically [105] classify the liver tissue into five stages: F0 - F4. F0 denotes normal liver tissues, and F1-F4 represent levels of liver fibrosis. Stages F1 and F2 were considered as early stages of liver fibrosis, and stages F3 and F4 were regarded as the late stages of liver fibrosis.

The collagen proportionate area (CPA) ratios are typically reported in conjunction with METAVIR classifications. Because detailed statistical analyses of CPA ratios were not available, we assumed that the CPA ratio follows a Gaussian distribution and subsequently derived its mean and standard deviation based on available data [108, 140] for each fibrosis stage, as shown in Table 6.2.

Table 6.2 Derived CPA values for 4 METAVIR stages (F1 to F4).

\begin{tabular}{ccccccccc}
\hline \hline \multirow{2}{*}{$\begin{array}{c}\text { METAVIR } \\
\text { stage }\end{array}$} & \multicolumn{2}{c}{ F1 } & \multicolumn{2}{c}{ F2 } & \multicolumn{2}{c}{ F3 } & \multicolumn{2}{c}{ F4 } \\
& Mean & STD & Mean & STD & Mean & STD & Mean & STD \\
\cline { 2 - 10 } CPA $(\%)$ & 4 & 1.7 & 9 & 5.5 & 13 & 7 & 19 & 10 \\
\hline \hline
\end{tabular}

The area fraction of each component and two-point correlation length $(\mathrm{CL})$ were calculated from all microscopic tissue distributions (Fig. 6.3c and d). Specifically, the twopoint CL was based on the two-point correlation function [141] used for the characterization of multiphase materials. Basically, the CL of $r$ represents a distance from 
which the probability of finding two points in the same (material) phase separated by a distance $r$ drops to a stable but low plateau (e.g. 0.2).

As shown in Table 6.3, to simulate one (METAVIR) fibrosis stage, three different CPA ratios were first used, and then simulation results were reweighted when the final simulation results were calculated. The weighting (see Table 6.3) was to ensure that the weighted CPA distribution under the fibrosis stage followed the assumed Gaussian distribution as shown in Table 6.2. Hence, 9 different realizations were used for each simulated liver fibrosis stage. Especially, each realization can generate 2160 data points of wave speed (i.e. SWS and SOS). Each CPA can generate $6480(2160 \times 3)$ data points. Then, we randomly select data points from each CPA based on weights shown in Table 6.3 to generate the final data points. To show how the presence of liver steatosis can affect the ultrasound measurements, moderate simulated liver steatosis (i.e. a fat area fraction of $50 \%$ [133]) was simultaneously simulated along with liver fibrosis following the signaling pathways described in Fig. 6.2, if necessary.

Table 6.3 A list of simulation configurations used in K-WAVE simulations. The weights listed were calculated so that the weighted CPA distribution of each fibrosis stage followed its respective Gaussian distribution (Table 6.2).

\begin{tabular}{cccccc}
\hline \hline $\begin{array}{c}\text { METAVIR } \\
\text { stage }\end{array}$ & $\begin{array}{c}\text { CPA } \\
(\%)\end{array}$ & $\begin{array}{c}\text { CL } \\
(\mathrm{mm})\end{array}$ & Realizations & $\begin{array}{c}\text { Total } \\
\text { Data Points }\end{array}$ & Weight \\
\hline \multirow{3}{*}{ F1 } & 3 & 0.6 & 3 & 6480 & $50.0 \%$ \\
& 5 & 0.9 & 3 & 6480 & $40.0 \%$ \\
& 8 & 0.9 & 3 & 6480 & $10.0 \%$ \\
\cline { 2 - 6 } F2 & 3 & 0.6 & 3 & 6480 & $18.4 \%$ \\
& 10 & 1.2 & 3 & 6480 & $52.2 \%$ \\
F3 & 3 & 1.3 & 3 & 6480 & $29.4 \%$ \\
\cline { 2 - 6 } & 13 & 0.6 & 3 & 6480 & $19.2 \%$ \\
& 1.3 & 3 & 6480 & $53.9 \%$ \\
& & & & &
\end{tabular}




\begin{tabular}{cccccc} 
& 21 & 1.4 & 3 & 6480 & $26.9 \%$ \\
\cline { 2 - 6 } F4 & 14 & 1.3 & 3 & 6480 & $33.3 \%$ \\
& 19 & 1.3 & 3 & 6480 & $38.1 \%$ \\
& 27 & 1.5 & 3 & 6480 & $28.6 \%$ \\
\hline \hline
\end{tabular}

\subsection{Validations Using Available Clinical Data}

The clinical SWS data (Table 6.4) were converted from the clinically-measured liver stiffness values reported in the literature using Eqn. (5.1):

$$
V_{s}=\sqrt{\frac{G}{\rho}}
$$

where $V_{S}, G$ and $\rho$ are SWS, shear modulus and, mass density, respectively.

Because of the raw SWS data from 3 peer-reviewed, (see Table 5.4) studies were not available, computer-synthesized SWS data derived from those three studies were generated as follows. Random SWS velocity data for each study were first generated by assuming that the SWS measurements in each study followed a Gaussian distribution. Then, synthesized SWS data from three studies were assembled such that the contribution of each clinical study was proportional to the number of patients available for the study. For instance, there were 2808 "computer-synthesized" SWS measurements for F1; among them, 151, 2435 and 222 were from Studies 1 to 3, respectively.

Table 6.4 Liver stiffness (Mean \pm one standard deviation) summarized from 3 published studies (hereafter referred as to Studies 1 to 3 ).

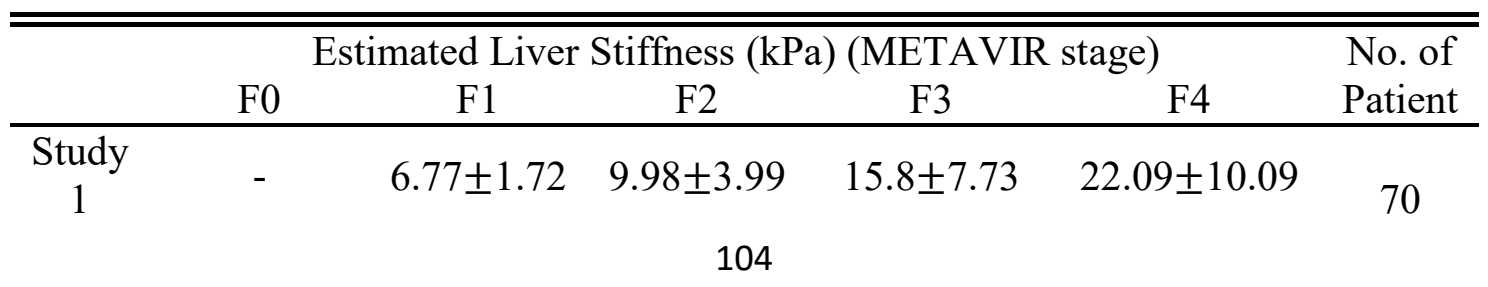




\begin{tabular}{ccccccc}
\hline $\begin{array}{c}{[120]} \\
\text { Study } \\
2\end{array}$ & $4.26 \pm 0.55$ & $5.51 \pm 1.5$ & $7.52 \pm 1.75$ & $10.44 \pm 3.48$ & $33.32 \pm 15.81$ & 1138 \\
{$[122]$} & & & & & & \\
Study & & & & & & \\
3 & - & $5.8 \pm 1.8$ & $11.3 \pm 6.8$ & $11.8 \pm 6$ & $23.4 \pm 16.5$ & 103 \\
{$[121]$} & & & & & & \\
\hline \hline
\end{tabular}

The clinical SOS data (Table 6.5) were obtained from a study by Boozari et al. [138] Boozari et al. used a commercial scanner (Zonare Medical Systems, Inc, Mountain View, CA, USA) equipped with a curvilinear array transducer (C5-2).

Table 6.5 SOS measurements reported in fibrotic livers [138].

\begin{tabular}{ccc}
\hline \hline METAVIR stage & F0-F3 & F4 \\
\hline SOS $(\mathrm{m} / \mathrm{s})$ & $1575 \pm 21$ & $1594 \pm 18$ \\
\hline \hline
\end{tabular}

\subsection{Parameters Involving in Systems Biology Simulations}

The above-mentioned SB simulations using SPARK consist of multiple sequential steps. In each step, all chemical and biological agents are updated. Major parameters are listed in Table 6.6 and those parameters can be largely divided into two categories: (1) parameters related to life cycle and (2) parameters defining mobility. More specifically, the life cycle was defined as the life span of chemical and biological agents and its unit was the simulation step. The mobility of biological and chemical agents was slightly different. The mobility of a biological agent denoted a distance traveled by the agent between two adjacent simulation steps and its unit was $\mathrm{mm} / \mathrm{step}$. The mobility of a chemical agent was a surrogate of the diffusion rate. Our rectilinear simulation domain was conventionally 
divided into squares. When a square was completely filled (100\%) with a chemical agent A at the $i^{\text {th }}$ simulation step, the mobility (or the diffusion rate) of A was the percentage occupancy of the agent $\mathrm{A}$ in the square's eight (8) immediate neighbors at the $(i+1)^{\text {th }}$ simulation step. Major parameters shown in Table 6.6 were inputs to the SB simulations, while collagen and lipid depositions were outputs.

Table 6.6. A list of selected parameters varied for sensitivity analysis of SB simulations using the stepwise regression method.

\begin{tabular}{|c|c|}
\hline Parameter (unit) & Value Range \\
\hline $\mathrm{CCL}_{4}$ Diffusion Rate (/step) & $\begin{array}{c}50 \%-90 \% \text { with an increment of } \\
5 \%\end{array}$ \\
\hline HMGB1 Diffusion Rate (/step) & $\begin{array}{c}10 \%-70 \% \text { with an increment of } \\
5 \%\end{array}$ \\
\hline TNF- $\alpha$ Diffusion Rate (/step) & $\begin{array}{c}10 \%-50 \% \text { with an increment of } \\
5 \%\end{array}$ \\
\hline TGF- $\beta 1$ Diffusion Rate (/step) & $\begin{array}{c}10 \%-50 \% \text { with an increment of } \\
5 \%\end{array}$ \\
\hline Fat Diffusion Rate (/step) & $\begin{array}{c}10 \%-50 \% \text { with an increment of } \\
5 \%\end{array}$ \\
\hline Fat Inhibit Probability & $\begin{array}{c}10 \%-50 \% \text { with an increment of } \\
5 \%\end{array}$ \\
\hline Stellate Move Speed (mm/step) & $\begin{array}{c}0.1-0.5 \text { with an increment of } \\
0.05\end{array}$ \\
\hline Hepatocyte Move Speed (mm/step) & $\begin{array}{c}0.1-0.5 \text { with an increment of } \\
0.05\end{array}$ \\
\hline Macrophage Move Speed (mm/step) & $\begin{array}{c}0.1-0.5 \text { with an increment of } \\
0.05\end{array}$ \\
\hline Hepatocyte Life Cycle (step) & $10-90$ with an increment of 10 \\
\hline
\end{tabular}

Stepwise regression [142] was used to determine influences of input parameters. Particularly, the stepwisefit function in Matlab (Mathworks, MA USA) added or removed relevant input parameters (see Table 6.6) based on their statistical significance in a multilinear regression fashion. A p-value of 0.05 was considered as statistically significant. Since 
the proposed SB simulations were stochastic in nature, for each combination of parameters used in the above-mentioned stepwise regression process, 5 realizations were performed and averaged outputs were reported in the Results Section.

\subsection{A Summary of Cases Simulated}

To test the performance of the proposed virtual liver tissue model, nine (9) different cases were simulated as summarized in Table 6.7.

Table 6.7. A summary of simulation conditions.

\begin{tabular}{|c|c|c|}
\hline $\begin{array}{l}\text { Case } \\
\text { No. }\end{array}$ & Purpose of Simulations & Simulation Conditions \\
\hline 1 & \multirow{4}{*}{$\begin{array}{l}\text { Qualitative Comparisons } \\
\text { to Histological } \\
\text { Characteristics }\end{array}$} & $\begin{array}{l}\text { Severe fibrosis without cirrhosis; simulation } \\
\text { steps }=200 ; \mathrm{CPA}=16 \% ;\end{array}$ \\
\hline 2 & & $\begin{array}{l}\text { Severe cirrhosis; simulation steps }=400 ; \mathrm{CPA}= \\
25 \% ;\end{array}$ \\
\hline 3 & & $\begin{array}{l}\text { Fatty lipid accumulation in the centrilobular } \\
\text { region; simulation steps }=430 ; \mathrm{CPA}=31 \% \text {; Fat } \\
=24 \% \text {; }\end{array}$ \\
\hline 4 & & $\begin{array}{l}\text { Distributed fat throughout the acinus; } \\
\text { simulation steps }=80 ; \mathrm{CPA}=13 \% \text {; Fat }=36 \% \text {; }\end{array}$ \\
\hline 5 & \multirow{2}{*}{$\begin{array}{l}\text { Parameters Analysis of } \\
\text { Systems Biology } \\
\text { Simulations }\end{array}$} & $\begin{array}{l}\text { Collagen deposition; simulation steps }=200 \text {; } \\
\text { Other parameters listed in Table } 6 \text {; }\end{array}$ \\
\hline 6 & & $\begin{array}{l}\text { Lipid deposition; simulation steps }=200 \text {; Other } \\
\text { parameters are given in Table } 6 ;\end{array}$ \\
\hline 7 & \multirow{2}{*}{$\begin{array}{l}\text { Effects of Fat Content and } \\
\text { Gaussian Kernel Size }\end{array}$} & $\begin{array}{l}\text { Fibrosis without steatosis at four different CPAs } \\
(13 \%, 15 \%, 21 \% \text { and } 26 \%) \text { in combinations } \\
\text { with different Gaussian kernel sizes; Other } \\
\text { parameters are given in Table } 3 \text {; }\end{array}$ \\
\hline 8 & & $\begin{array}{l}\text { Fibrosis with steatosis }(50 \%) \text { at two different } \\
\text { CPAs }(21 \% \text { and } 26 \%) \text { with different Gaussian } \\
\text { kernel sizes; Other parameters are given in } \\
\text { Table 3; }\end{array}$ \\
\hline 9 & $\begin{array}{l}\text { Comparisons to SWS and } \\
\text { SOS Measurements }\end{array}$ & $\begin{array}{l}\text { Fibrotic tissues (F1-F4) without steatosis; Other } \\
\text { details are listed in Table 3; F1 with a Gaussian } \\
\text { kernel sizes were } 0.1 \mathrm{~mm}, 0.2 \mathrm{~mm}, 0.2 \mathrm{~mm} \text { and } \\
0.4 \mathrm{~mm} \text { for F1-F4, respectively. }\end{array}$ \\
\hline
\end{tabular}




\subsection{Data Analysis}

A lateral time-to-peak (TTP) algorithm [44] was adopted to estimate wave speeds (i.e. both SWS and SOS) along the respective wave propagation directions. The processing kernel length was $0.4 \mathrm{~mm}$. To verify the kernel length sensitivity for the simulated wave speed, our results were reprocessed using a processing kernel length of $1.2 \mathrm{~mm}$. We found that the means of wave speed remained to be the same but the variances were typically reduced by approximately 5\%. Then, a direction filter [103] was applied to the simulated wave data to remove some wave interferences. Finally, the simulated shear/compressional wave speed data can be obtained. More details regarding the calculation of wave speed using simulated data can be found in our prior work [139].

\subsection{Qualitative Comparisons to Histological Characteristics (Cases 1-4)}

Fig. 6.5a (Case 1) shows an example of severe fibrosis without cirrhosis. The histology slide showing growing fibrosis along the septa (see the top image in Fig. 6.5a) was obtained from a rat experiment, in which intraperitoneal administration of $\mathrm{CCl}_{4}$ lasted for 8 weeks [143]. Fig. 6.5b (Case 2) depicts an example of severe cirrhosis (F4) in a human subject [144].

Figs. 6.6a (Case 3) and 6.6b (Case 4) show the fat accumulation in the centrilobular region and distributed fatty lipids throughout the acinus, respectively. Particularly, Case 3 had histologic features of liver fibrosis in non-alcoholic steatohepatitis (NASH) and the relevant histological slide (see the top image in Fig. 6.6a) demonstrates that macrovesicular steatosis and fibrosis were near the central vein of the hepatic lobule [145]. Case 4 (see the histological slide in Fig. 6.6b) contained classic histologic findings in the alcoholic liver 
disease, in which diffuse macrovesicular steatosis was associated with perisinusoidal fibrosis and lobular necroinflammatory activity [146]. Overall, histological features visualized from SB-simulated liver fibrosis with/without steatosis were qualitatively similar to those displayed in actual histological slides.

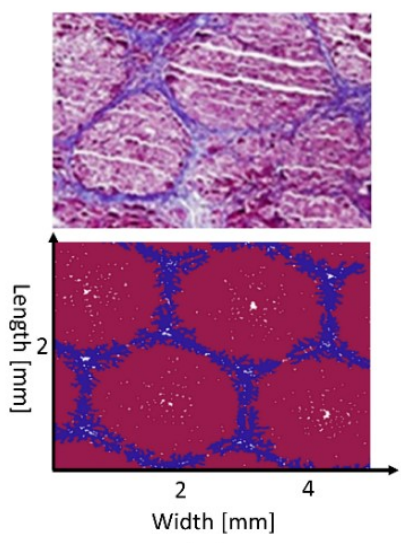

(a)

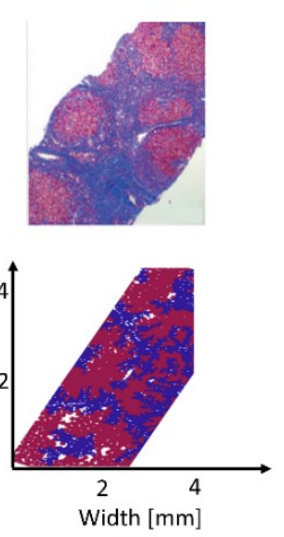

(b)

Figure 6.5. Examples showing comparisons between histology and simulated virtual liver tissues without steatosis: (a) Severe fibrosis without cirrhosis (Case 1; equivalent to F3) [143] and (b) Severe cirrhosis (Case 2; equivalent to F4) [144]. Histology slides and SB-simulated liver tissues are listed in the first and second rows, respectively. The trichrome stain was used.

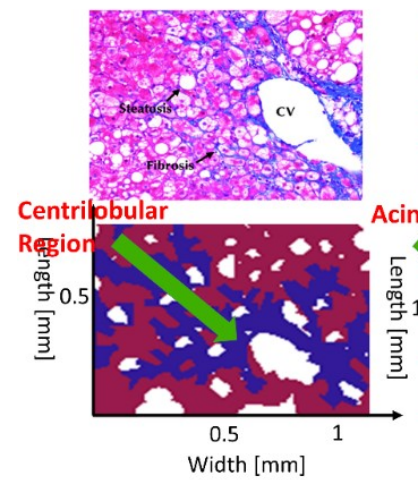

(a)

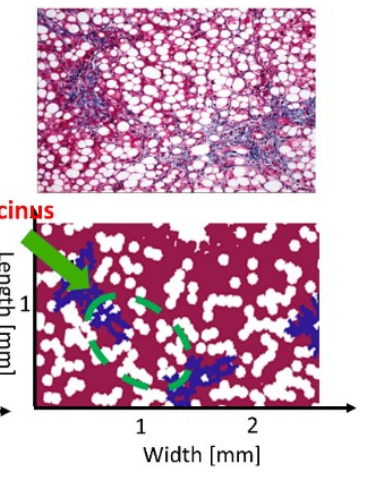

(b)

Figure 6.6. Examples showing comparisons between histology and SB-simulated liver tissues with steatosis: (a) Fatty lipid accumulation in the centrilobular region (Case 3) 
[145] and (b) Distributed fat throughout the acinus (Case 4) [146]. Histology slides and SB-simulated liver tissues are listed in the first and second rows, respectively. The trichrome stain was used. In (a), the green arrow represents the centrilobular region. In (b), the green ellipse represents acinus.

\subsection{Parameters Analysis Using Stepwise Regression (Cases 5-6)}

Recall that the collagen and lipid contents were statistically analyzed by the stepwise regression method [142]. Two general scenarios were considered: (1) the collagen (Table 6.8) and lipid (Table 6.9) depositions were studied independently; (2) the dynamics between the liver fibrosis and steatosis was investigated together (Table 6.10) though the output of interest was still the collagen content (see Table 6.10), given the fact our primary focus is on fibrotic liver tissues.

As summarized in Table 6.8, if only the liver fibrosis was simulated through SB simulations (Fig. 6.2), only the p-values of $\mathrm{CCL}_{4}$ and $\mathrm{HMGB}_{1}$ diffusion rate were below 0.05. This observation indicates that only these two agents, $\mathrm{CCL}_{4}$ and HMGB1, affected the collagen deposition in a statistically significant manner. Similarly, results in Table 6.9 indicate that the $\mathrm{p}$-values of fat diffusion rate and fat inhibit probability were below 0.05 , meaning that these two parameters can significantly influence the fat deposition. How the diffusion rates of $\mathrm{CCL}_{4}$ and $\mathrm{HMGB} 1$ quantitatively influenced the CPA is displayed in Fig. 6.7a (Case 5), while the influences of the diffusion rate of fat and the death probability of lipid cells to the fat deposition are depicted in Fig. 6.7b (Case 6). 


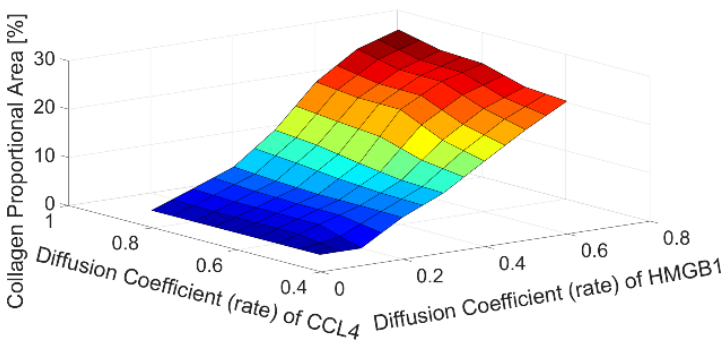

(a)

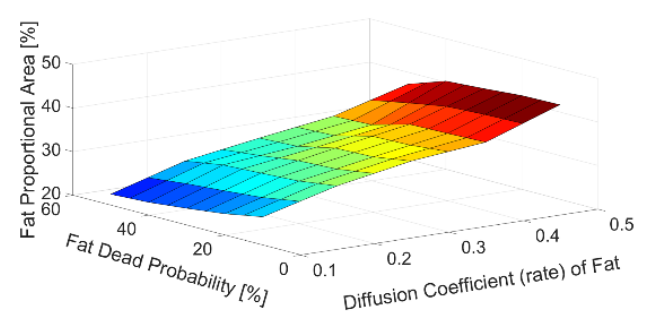

(b)

Figure 6.7. Plots of (a) the collagen deposition with the respect of the $\mathrm{CCL}_{4}$ diffusion rate and the HMGB1 diffusion rate (Case 5) and (b) the lipid deposition with the respect of the death probability of lipid cells and fat diffusion rate (Case 6).

Table 6.8. P-values of parameters used during the SB simulations of the progression of liver fibrosis without steatosis. P-values were obtained by the stepwise regression.

\begin{tabular}{ccc}
\hline \hline Inputs & Output & p-value \\
\hline CCL4 Diffusion Rate & & $1.7266 \mathrm{e}-10$ \\
HMGB1 Diffusion Rate & & $1.7266 \mathrm{e}-10$ \\
TNF- $\alpha$ Diffusion Rate & & 0.6298 \\
TGF- $\beta$ 1 Diffusion Rate & Collagen Deposition & 0.7836 \\
Stellate Move Speed & & 0.9816 \\
Hepatocyte Move Speed & & 0.9894 \\
Hepatocyte Life Cycle & 0.6489 \\
\hline \hline
\end{tabular}

When the simulations of liver fibrosis and steatosis were combined, we found that the collagen deposition process was almost independently associated with the liver steatosis, as indicated by the p-values in Table 6.10.

Table 6.9. P-values of parameters used during the SB simulations of the progression of liver steatosis. P-values were obtained by the stepwise regression.

\begin{tabular}{ccc}
\hline \hline Inputs & Output & p-value \\
\hline Fat Diffusion Rate & & $1.6322 \mathrm{e}-10$ \\
Fat Inhibit Probability & Lipid Deposition & $1.6328 \mathrm{e}-10$ \\
TNF- $\alpha$ Diffusion Rate & & 0.7851
\end{tabular}


TGF- $\beta 1$ Diffusion Rate

0.8131

Macrophage Move Speed

0.9382

HMGB1- $\beta$ Diffusion Rate

Table 6.10. P-values of parameters used during the SB simulations of the progression of combined liver fibrosis and steatosis. P-values were obtained by the stepwise regression.

\begin{tabular}{|c|c|c|}
\hline Inputs & Output & p-value \\
\hline CCL4 Diffusion Rate & \multirow{10}{*}{ Collagen Deposition } & $1.6573 \mathrm{e}-10$ \\
\hline HMGB1- $\beta$ Diffusion Rate & & $1.6587 \mathrm{e}-10$ \\
\hline TNF- $\alpha$ Diffusion Rate & & 0.7185 \\
\hline TGF- $\beta 1$ Diffusion Rate & & 0.8327 \\
\hline Stellate Move Speed & & 0.8894 \\
\hline Hepatocyte Move Speed & & 0.9869 \\
\hline Hepatocyte Life Cycle & & 0.7489 \\
\hline Macrophage Move Speed & & 0.9576 \\
\hline Fat Diffusion Rate & & 0.8465 \\
\hline Fat Inhibit Probability & & 0.6486 \\
\hline
\end{tabular}

\subsection{Comparisons to Wave Speed Measurements (Cases 7-9)}

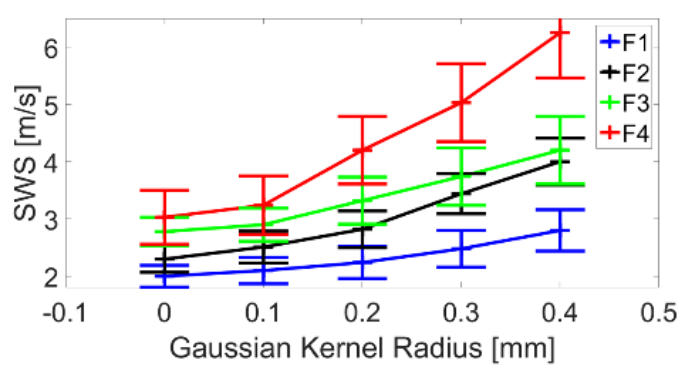

(a)

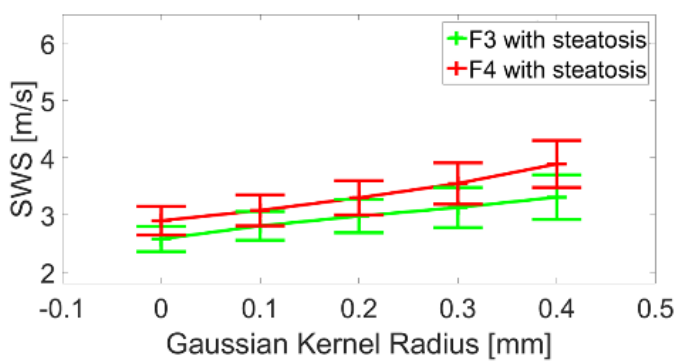

(b)

Figure 6.8. Plots showing SWS values influenced by (a) the Gaussian kernel size and (b) the presence of simulated lipid accumulation. In (a), the CPA values were $13 \%, 15 \%$, $21 \%$ and $26 \%$ for $\mathrm{F} 1-\mathrm{F} 4$, respectively (Case 7 ). In (b), roughly $50 \%$ fatty lipid accumulation was simulated both for F3 (CPA of 21\%) and F4 (CPA of 26\%) stages (case 8). In (a) and (b), the error bar represents one standard deviation. 
Fig. 6.8a (Case 7) shows that, with the increase of CPA, the estimated SWS value increased. It is worth noting that, without the simulation of collagen cross-linking, the estimated SWS could only be elevated to approximately $4.5 \mathrm{~m} / \mathrm{s}$, even when the CPA reached 50\%. To match the elevated SWS values from stage F1 (approximately $2.5 \mathrm{~m} / \mathrm{s}$ ) to F4 (approximately $6 \mathrm{~m} / \mathrm{s}$ ) reported in the literature [120-122], we found that the kernel sizes of $0.1 \mathrm{~mm}, 0.2 \mathrm{~mm}, 0.2 \mathrm{~mm}$ and $0.4 \mathrm{~mm}$ were most appropriate for $\mathrm{F} 1$ to $\mathrm{F} 4$, respectively. Consequently, we fixed those kernel sizes for subsequent wave simulations.

Interestingly, SWS values did not differ significantly in simulated fibrotic tissues (F3 and F4) in the presence of significant (50\%) steatosis, as shown in Fig. 6.8b (Case 8). Simulated representative micromechanical models (Case 8) are displayed in Fig. 6.9 and their related parameters are summarized in Table 6.11. 

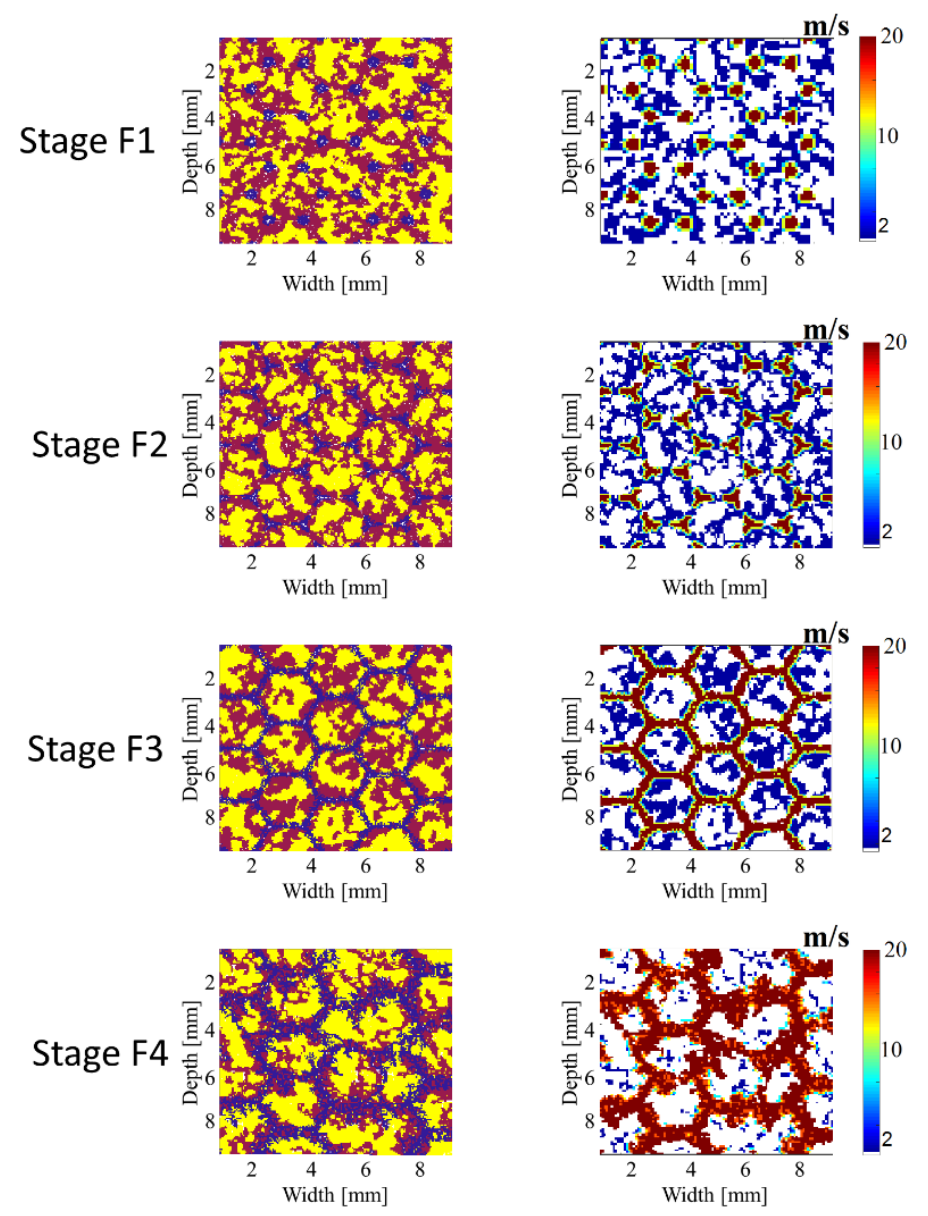

(a)

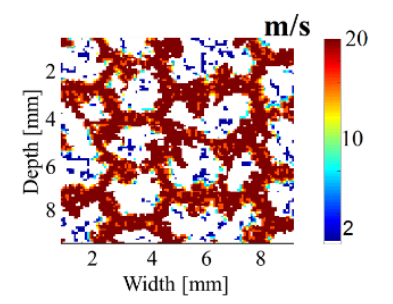

(b)

Figure 6.9. Four representative realizations of fibrotic liver tissues (i.e. F1-F4) with steatosis. Images in the (a) first and (b) second columns represent simulated histological characteristics and corresponding SWS distributions, respectively. Overall, the red, yellow and blue represent normal tissue, fat, and fibrosis, respectively, except that the white color in column (b) indicates the fat content for better visualization. In column (b), the color bar indicates SWS $(\mathrm{m} / \mathrm{s})$.

Table 6.11 A list of resultant tissue compositions of simulated fibrotic liver tissues illustrated in Figures 9a-b.

\begin{tabular}{ccccc}
\hline \hline & Fibrosis $(\%)$ & Fat $(\%)$ & $\begin{array}{c}\text { Normal Tissue } \\
(\%)\end{array}$ & $\begin{array}{c}\text { Kernel Size } \\
(\mathrm{mm})\end{array}$ \\
\hline F1 & 7.8 & 50.0 & 42.2 & 0.1
\end{tabular}




\begin{tabular}{ccccc} 
F2 & 9.6 & 50.0 & 40.4 & 0.2 \\
F3 & 23.3 & 50.0 & 26.7 & 0.2 \\
F4 & 34.5 & 50.0 & 15.5 & 0.4 \\
\hline \hline
\end{tabular}

Results simulated under the Case 9 are displayed in Fig. 6.10. In Fig. 6.10a, we found that the estimated SWS values from K-WAVE simulations increased with the advance of liver fibrosis from F1-F4. Also, the virtual SWS estimates from the K-WAVE simulated data largely overlapped with data available in 3 peer-reviewed studies [120-122]. In Fig. 6.10b, the probability density functions (PDFs) of the virtual SWS estimates are displayed together with PDFs generated from the computer-synthesized clinical data (subsection 3 of the Materials Section). Four PDFs of virtual SWS data were in good agreement with PDFs of "computer-synthesized" clinical SWS data. The overlaps among 4 pairs of PFDs all exceeded 90\%. In Fig. 6.10c, the estimated SOS values also largely overlapped the range of the SOS values clinically measured.

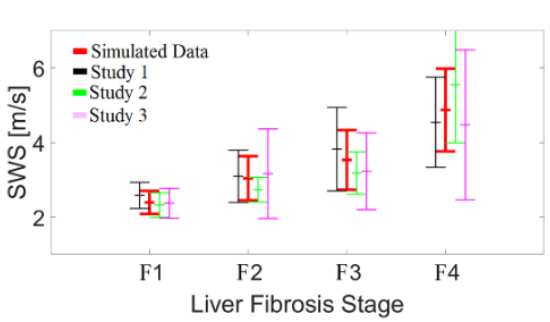

(a)

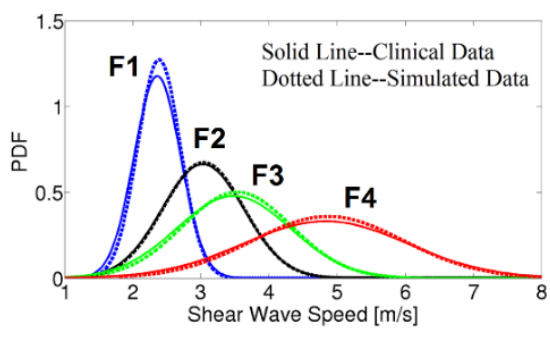

(b)

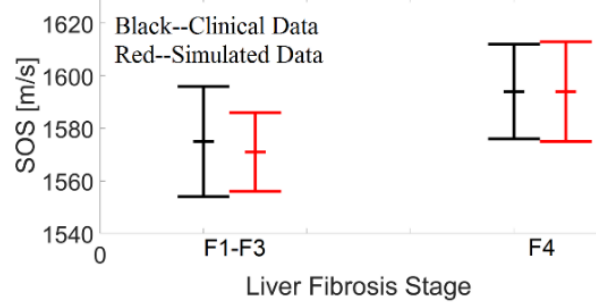

(c) 
Figure 6.10. Plots comparing virtual simulation data with clinically measured data for Case 9: (a) SWS values, (b) PDFs of SWS distributions, and (c) SOS values. In (a) and (c), the error bar represents one standard deviation.

\subsection{Discussion}

One of our contributions is the inclusion of liver steatosis in systems biology-based simulations. The simulation of $\mathrm{CCL}_{4}$ induced liver injuries was inherited from the work of Dutta-Moscato et al. and they have already compared their work to the histological results in a rat model [60]. In this study, we further extended the comparison to the combination of liver steatosis and fibrosis using human pathology. Our preliminary results (Figs. 6.5 and 6.6) indicated that the systems biology-simulated collagen and fat depositions were qualitatively similar to histological characteristics. This is mainly due to the fact that the $\mathrm{CCL}_{4}$ model resembles the histology of human liver fibrosis well [147]. More quantitative comparisons to human pathology under various conditions are needed to further validate the proposed signaling pathways (see Fig. 6.2).

Another contribution is to use the proposed virtual tissue model for ultrasoundbased tissue characterization. To our knowledge, this is the first biology-based micromechanical model designed for ultrasound tissue characterization. Although the virtual liver tissue model is simplistic, the preliminary results are encouraging. Our results suggested that the results from the K-WAVE simulations were able to reproduce SWS and SOS measurements reported in the literature (Figs. 6.10a-c). It is interesting to note that, in order to match SWS values reported in the clinical literature, the Gaussian kernel size had to be increased from $0.1 \mathrm{~mm}$ for F1 to $0.4 \mathrm{~mm}$ for F4. Physically, this is an indication 
of increasing collagen cross-linking with the progression of liver fibrosis. This finding seemed consistent with the biology literature $[148,149]$. Furthermore, results shown in Fig. 6.8b were consistent with a clinical study where SWE may fail to detect fibrosis in significant steatosis background [34].

Results in Table 6.10 indicated that the interactions between the simulated liver fibrosis and steatosis were not strong. This observation is actually comparable to findings reported in the clinical literature [150]. However, more work is needed because the interactions between the liver fibrosis and the steatosis are complicated and currently not fully understood. For instance, the effects of TNF- $\alpha$ on lipid metabolism alone have at least 5 different pathways [151]. Our future plan is to implement more knowledge-based rules related to liver fibrosis and lipid metabolism into the proposed framework in order to further explore this line of research.

Overall, the proposed research has expanded the paradigm of virtual imaging simulation [84, 152]. Currently, virtual imaging simulations utilize computer-aided engineering software (i.e. geometry modeling and numerical modeling of image physics) to evaluate novel imaging methods without making a physical/hardware prototype. By the inclusion of the systems biology, it is possible that we can continuously simulate imaging of pathological changes due to clinical interventions. In the future, applications could include the investigation of the feasibility of monitoring of drug treatment of liver fibrosis or chemotherapy of breast cancers.

There are limitations. First, the virtual tissue model was purely elastic as the first approximation. Liver tissues are indeed viscoelastic and therefore, SWS and SOS 
measurements may be frequency-dependent [129]. Second, rigorous validations using an animal model will enable us to tune rules related to the systems biology simulations and conversions to mechanical properties. Now the feasibility has been successfully tested and both limitations will be addressed in a follow-up study.

\subsection{Conclusion}

In this study, we proposed a systems biology-inspired approach directly linking the tissue microstructures, compositions and architectures of fibrotic livers to related ultrasound measurements such as SWS and SOS. We demonstrated that those virtual fibrotic liver tissues can reflect spatial characteristics of relevant histology. Their mechanical characteristics and wave measurements were in good agreement with data reported in the literature. 


\section{Chapter 7. Summary and Future Work}

\subsection{Summary}

To improve the confidence in UE measurements or image interpretation for breast lesion differentiation and staging liver fibrosis, my thesis developed a computational simulation platform integrating open-source software packages with novel complex numerical tissue models to better to simulate SE and acoustic radiation force-based SWE within the heterogeneous tissue.

The proposed simulation platform is innovative. It is the first time that complex, heterogeneous but known tissue media was adapted into an advanced UE simulation platform. The gaps among the open-source software packages were filled, which can act like a pipeline. To show the capability and innovation of the proposed simulation platform, a complex numerical breast model was built in chapter 2. Then, clinical ultrasound techniques for breast cancer differentiation were tested on the proposed breast model: chapter 2 (i.e. strain elastography) and chapter 3 (i.e. shear wave elastography).

To improve the understanding of staging liver fibrotic tissue in UE, intrinsic variations of SWS in the liver fibrotic tissue were statistically analyzed using a stochastic tissue model in chapter 4. Also, group SWS dispersion caused by elastic scattering was analyzed in chapter 5. It is the first time that a probability-based assessment of SWS was applied to SWE data analysis. The theoretical analysis methods described in chapters 4 and 5 can be extended to the SWS data obtained from the proposed simulation platform. 
Finally, a "biologically relevant" liver tissue model was built in chapter 6 , using a System Biology method, to relate UE measurements to the underlying structures of the liver fibrosis tissue with/without steatosis. Compared with the simple tissue-mimicking models and numerical models used in the elastography literature, it is the first time that "biologically relevant" soft tissue models were used in UE simulations. This method described in chapter 6 can be extended to the proposed simulation platform to increase the multi-scale property of the complex numerical model.

\subsection{Future Work}

In the future, clinical applications of staging liver fibrotic tissue will be simulated by the proposed computational platform. To increase the reality and heterogeneity of the numerical liver tissue model, System Biology methods will be used to generate additional "biologically relevant" small structures in the model. The SWS data simulated from the proposed simulation platform will be analyzed statistically. 


\section{References}

[1] T. Shiina, K. R. Nightingale, M. L. Palmeri, T. J. Hall, J. C. Bamber, R. G. Barr, et al., "Wfumb Guidelines and Recommendations for Clinical Use of Ultrasound Elastography: Part 1: Basic Principles and Terminology," Ultrasound in Medicine and Biology, vol. 41, pp. 1126-1147, May 2015.

[2] J. Bamber, D. Cosgrove, C. F. Dietrich, J. Fromageau, J. Bojunga, F. Calliada, et al., "EFSUMB Guidelines and Recommendations on the Clinical Use of Ultrasound Elastography. Part 1: Basic Principles and Technology," Ultraschall in Der Medizin, vol. 34, pp. 169-184, Apr 2013.

[3] K. Nightingale, "Acoustic Radiation Force Impulse (ARFI) Imaging: a Review," Curr Med Imaging Rev, vol. 7, pp. 328-339, Nov 12011.

[4] J. L. Gennisson, T. Deffieux, M. Fink, and M. Tanter, "Ultrasound elastography: principles and techniques," Diagn Interv Imaging, vol. 94, pp. 487-95, May 2013.

[5] D. Cosgrove, F. Piscaglia, J. Bamber, J. Bojunga, J. M. Correas, O. H. Gilja, et al., "EFSUMB Guidelines and Recommendations on the Clinical Use of Ultrasound Elastography. Part 2: Clinical Applications," Ultraschall in Der Medizin, vol. 34, pp. 238-253, Jun 2013.

[6] ACS, "Breast Cancer Facts \& Figures 2015-2016," American Cancer Society, 2016.

[7] K. C. Oeffinger, E. T. H. Fontham, R. Etzioni, A. Herzig, J. S. Michaelson, Y. C. T. Shih, et al., "Breast Cancer Screening for Women at Average Risk 2015 Guideline Update From the American Cancer Society," Jama-Journal of the American Medical Association, vol. 314, pp. 1599-1614, Oct 202015.

[8] L. Tabar, B. Vitak, T. H. Chen, A. M. Yen, A. Cohen, T. Tot, et al., "Swedish two-county trial: impact of mammographic screening on breast cancer mortality during 3 decades," Radiology, vol. 260, pp. 658-63, Sep 2011.

[9] W. A. Berg, L. Gutierrez, M. S. NessAiver, W. B. Carter, M. Bhargavan, R. S. Lewis, et al., "Diagnostic accuracy of mammography, clinical examination, US, and MR imaging in preoperative assessment of breast cancer," Radiology, vol. 233, pp. 830-849, Dec 2004.

[10] P. T. Huynh, A. M. Jarolimek, and S. Daye, "The false-negative mammogram," Radiographics, vol. 18, pp. 1137-1154, Sep-Oct 1998.

[11] J. Ophir, I. Cespedes, H. Ponnekanti, Y. Yazdi, and X. Li, "Elastography: a quantitative method for imaging the elasticity of biological tissues," Ultrasonic Imaging, vol. 13, pp. 111-34, 1991.

[12] T. J. Hall, Y. N. Zhu, and C. S. Spalding, "In vivo real-time freehand palpation imaging," Ultrasound in Medicine and Biology, vol. 29, pp. 427-435, 2003.

[13] M. Odonnell, A. R. Skovoroda, B. M. Shapo, and S. Y. Emelianov, "Internal Displacement and Strain Imaging Using Ultrasonic Speckle Tracking," Ieee Transactions on Ultrasonics Ferroelectrics and Frequency Control, vol. 41, pp. 314-325, May 1994.

[14] E. S. Burnside, T. J. Hall, A. M. Sommer, G. K. Hesley, G. A. Sisney, W. E. Svensson, et al., "Differentiating benign from malignant solid breast masses with US strain imaging," Radiology, vol. 245, pp. 401-410, Nov 2007. 
[15] X. Gong, Q. H. Xu, Z. L. Xu, P. Xiong, W. L. Yan, and Y. Z. Chen, "Real-time elastography for the differentiation of benign and malignant breast lesions: a meta-analysis," Breast Cancer Research and Treatment, vol. 130, pp. 11-18, Nov 2011.

[16] M. L. Palmeri and K. R. Nightingale, "Acoustic radiation force-based elasticity imaging methods," Interface Focus, vol. 1, pp. 553-64, Aug 62011.

[17] B. Liu, Y. Zheng, G. Huang, M. Lin, Q. Shan, Y. Lu, et al., "Breast Lesions: Quantitative Diagnosis Using Ultrasound Shear Wave Elastography\&\#x2014;A Systematic Review and Meta-Analysis," Ultrasound in Medicine and Biology, vol. 42, pp. 835-847, 2016.

[18] H. Zhao, P. F. Song, M. W. Urban, R. R. Kinnick, M. Yin, J. F. Greenleaf, et al., "Bias Observed in Time-of-Flight Shear Wave Speed Measurements Using Radiation Force of a Focused Ultrasound Beam," Ultrasound in Medicine and Biology, vol. 37, pp. 1884-1892, Nov 2011.

[19] T. J. Hall, A. Milkowski, E. Garra, P. Carson, M. Palmeri, K. Nightingale, et al., "RSNA/QIBA: Shear wave speed as a biomarker for liver fibrosis staging," in 2013 IEEE International Ultrasonics Symposium (IUS), 2013, pp. 397-400.

[20] A. Birbrair, T. Zhang, D. C. Files, S. Mannava, T. Smith, Z. M. Wang, et al., "Type-1 pericytes accumulate after tissue injury and produce collagen in an organ-dependent manner," Stem Cell Research \& Therapy, vol. 5, Nov 62014.

[21] R. G. Barr, G. Ferraioli, M. L. Palmeri, Z. D. Goodman, G. Garcia-Tsao, J. Rubin, et al., "Elastography Assessment of Liver Fibrosis: Society of Radiologists in Ultrasound Consensus Conference Statement," Radiology, vol. 276, pp. 845-61, Sep 2015.

[22] P. Mofrad, M. J. Contos, M. Haque, C. Sargeant, R. A. Fisher, V. A. Luketic, et al., "Clinical and histologic spectrum of nonalcoholic fatty liver disease associated with normal ALT values," Hepatology, vol. 37, pp. 1286-92, Jun 2003.

[23] J. Parkes, I. N. Guha, P. Roderick, and W. Rosenberg, "Performance of serum marker panels for liver fibrosis in chronic hepatitis C," J Hepatol, vol. 44, pp. 462-74, Mar 2006.

[24] M. Friedrich-Rust, W. Rosenberg, J. Parkes, E. Herrmann, S. Zeuzem, and C. Sarrazin, "Comparison of ELF, FibroTest and FibroScan for the non-invasive assessment of liver fibrosis," BMC Gastroenterol, vol. 10, p. 103, 2010.

[25] E. Carey and W. D. Carey, "Noninvasive tests for liver disease, fibrosis, and cirrhosis: Is liver biopsy obsolete?," Cleve Clin J Med, vol. 77, pp. 519-27, Aug 2010.

[26] A. P. Sarvazyan, O. V. Rudenko, S. D. Swanson, J. B. Fowlkes, and S. Y. Emelianov, "Shear wave elasticity imaging: a new ultrasonic technology of medical diagnostics," Ultrasound Med Biol, vol. 24, pp. 1419-35, Nov 1998.

[27] K. Nightingale, S. McAleavey, and G. Trahey, "Shear-wave generation using acoustic radiation force: in vivo and ex vivo results," Ultrasound Med Biol, vol. 29, pp. 1715-23, Dec 2003.

[28] J. Bercoff, M. Tanter, and M. Fink, "Supersonic shear imaging: a new technique for soft tissue elasticity mapping," IEEE Trans Ultrason Ferroelectr Freq Control, vol. 51, pp. 396-409, Apr 2004. 
[29] L. Sandrin, B. Fourquet, J. M. Hasquenoph, S. Yon, C. Fournier, F. Mal, et al., "Transient elastography: a new noninvasive method for assessment of hepatic fibrosis," Ultrasound Med Biol, vol. 29, pp. 1705-13, Dec 2003.

[30] G. Ferraioli, C. Filice, L. Castera, B. I. Choi, I. Sporea, S. R. Wilson, et al., "Wfumb Guidelines and Recommendations for Clinical Use of Ultrasound Elastography: Part 3: Liver," Ultrasound in Medicine and Biology, vol. 41, pp. 1161-1179, May 2015.

[31] G. Ferraioli, P. Parekh, A. B. Levitov, and C. Filice, "Shear wave elastography for evaluation of liver fibrosis," J Ultrasound Med, vol. 33, pp. 197-203, Feb 2014.

[32] F. J. Castera L, Bernard P H, Carvalho F, Allaix D, Merrouche W, Couzigou P and de Ledinghen $\mathrm{V}$, "Pitfalls of liver stiffness measurement: a 5 -year prospective study of 13,369 examinations," Hepatology, pp. 828-35, 2010.

[33] I. Goldschmidt, C. Streckenbach, C. Dingemann, E. D. Pfister, A. di Nanni, A. Zapf, et al., "Application and limitations of transient liver elastography in children," J Pediatr Gastroenterol Nutr, vol. 57, pp. 109-13, Jul 2013.

[34] A. Wieckowska and A. E. Feldstein, "Diagnosis of nonalcoholic fatty liver disease: invasive versus noninvasive," Semin Liver Dis, vol. 28, pp. 386-95, Nov 2008.

[35] D. C. Rockey and D. M. Bissell, "Noninvasive measures of liver fibrosis," Hepatology, vol. 43, pp. S113-20, Feb 2006.

[36] A. M. Timothy J Hall, Brian Garra, Paul Carson, Mark Palmeri, Kathy Nightingale, Ted Lynch, Abdullah Alturki, Michael Andre, Stephane Audiere, Jeffery Bamber, Richard Barr, Jeremy Bercoff, Jessica Bercoff, Miguel Bernal, Javier Brum, Huan Wee Chan, Shigao Chen, Claude Cohen-Bacrie, Mathieu Couade, Allison Daniels, Ryan DeWall, Jonathan Dillman, Richard Ehman, SF Franchi-Abella, Jeremie Fromageau, Jean-Luc Gennisson, Jean Pierre Henry, Nikolas Ivancevich, Jan Kalin, Sarah Kohn, Jennifer Kugel, Ken Lee, NL Liu, Thanasis Loupas, Joan Mazernik, Stephen McAleavey, Veronique Miette, Stephen Metz, BM Morel, Thomas Nelson, Eric Nordberg, Jennifer Oudry, Monali Padwal, Ned Rouze, Anthony Samir, Laurent Sandrin, Janet Schaccitti, Cedric Schmitt, Vijay Shamdasani, Pehngfei Song, Pamela Switalski, Michael Wang, Keith Wear, Hua Xie, Heng Zhao, "RSNA/QIBA: Shear wave speed as a biomarker for liver fibrosis staging," Ultrasonics Symposium (IUS), 2013 IEEE International2013 (unpublished). 2013.

[37] R. Collier, "Rapidly rising clinical trial costs worry researchers," CMAJ, vol. 180, pp. 277-8, Feb 32009.

[38] E. L. Madsen, M. A. Hobson, H. R. Shi, T. Varghese, and G. R. Frank, "Stability of heterogeneous elastography phantoms made from oil dispersions in aqueous gels," Ultrasound in Medicine and Biology, vol. 32, pp. 261-270, Feb 2006.

[39] M. A. Hobson, E. L. Madsen, G. R. Frank, J. Jiang, H. Shi, T. J. Hall, et al., "Anthropomorphic phantoms for assessment of strain imaging methods involving saline-infused sonohysterography," Ultrasound Med Biol, vol. 34, pp. 1622-37, Oct 2008. 
[40] I. M. de Carvalho, R. L. Q. Basto, and A. F. C. Infantosi, "Breast Ultrasound Imaging Phantom to Mimic Malign Lesion Characteristics," Physics Procedia, vol. 3, pp. 421-426, 2010.

[41] F. S. Azar, D. N. Metaxas, and M. D. Schnall, "A deformable finite element model of the breast for predicting mechanical deformations under external perturbations," Acad Radiol, vol. 8, pp. 965-75, Oct 2001.

[42] A. Samani, J. Bishop, M. J. Yaffe, and D. B. Plewes, "Biomechanical 3-D finite element modeling of the human breast using MRI data," IEEE Trans Med Imaging, vol. 20, pp. 271-9, Apr 2001.

[43] C. M. Hsu, M. L. Palmeri, W. P. Segars, A. I. Veress, and J. T. Dobbins, 3rd, "Generation of a suite of 3D computer-generated breast phantoms from a limited set of human subject data," Med Phys, vol. 40, p. 043703, Apr 2013.

[44] M. L. Palmeri, M. H. Wang, J. J. Dahl, K. D. Frinkley, and K. R. Nightingale, "Quantifying hepatic shear modulus in vivo using acoustic radiation force," Ultrasound in Medicine and Biology, vol. 34, pp. 546-558, Apr 2008.

[45] D. W. Park, "3D Ultrasound Shear Wave Imaging Simulation of Heterogeneous Tissue," Journal of Electrical Engineering \& Technology, vol. 11, pp. 1486-1491, Sep 2016.

[46] J. Q. Mo, H. Xu, B. Qiang, H. Giambini, R. Kinnick, K. N. An, et al., "Bias of shear wave elasticity measurements in thin layer samples and a simple correction strategy," Springerplus, vol. 5, Aug 122016.

[47] A. Samani, J. Bishop, M. J. Yaffe, and D. B. Plewes, "Biomechanical 3-D finite element modeling of the human breast using MRI data," Ieee Transactions on Medical Imaging, vol. 20, pp. 271-279, Apr 2001.

[48] F. Kallel, C. D. Prihoda, and J. Ophir, "Contrast-transfer efficiency for continuously varying tissue moduli: simulation and phantom validation," Ultrasound Med Biol, vol. 27, pp. 1115-25, Aug 2001.

[49] M. H. Wang, M. L. Palmeri, N. C. Rouze, K. R. Nightingale, and M. A. Hobson, "Investigating the effects of viscosity on focused, impulsive, acoustic radiation force induced shear wave morphology," in 2008 IEEE Ultrasonics Symposium, 2008, pp. 647-650.

[50] M. L. Palmeri, N. C. Rouze, M. H. Wang, X. Ding, and K. R. Nightingale, "Quantifying the impact of shear wavelength and kernel size on shear wave speed estimation," in 2010 IEEE International Ultrasonics Symposium, 2010, pp. 13-16.

[51] K. H. Lee, B. A. Szajewski, Z. Hah, K. J. Parker, and A. M. Maniatty, "Modeling shear waves through a viscoelastic medium induced by acoustic radiation force," International Journal for Numerical Methods in Biomedical Engineering, vol. 28, pp. 678-696, Jun-Jul 2012.

[52] A. Caenen, D. Shcherbakova, B. Verhegghe, C. Papadacci, M. Pernot, P. Segers, et al., "A versatile and experimentally validated finite element model to assess the accuracy of shear wave elastography in a bounded viscoelastic medium," IEEE Trans Ultrason Ferroelectr Freq Control, vol. 62, pp. 439-50, Mar 2015.

[53] D. W. Park, "Ultrasound Shear Wave Simulation of Breast Tumor Using Nonlinear Tissue Elasticity," Computational and Mathematical Methods in Medicine, 2016. 
[54] S. L. Lipman, N. C. Rouze, M. L. Palmeri, and K. R. Nightingale, "Evaluating the Improvement in Shear Wave Speed Image Quality Using Multidimensional Directional Filters in the Presence of Reflection Artifacts," Ieee Transactions on Ultrasonics Ferroelectrics and Frequency Control, vol. 63, pp. 1049-1063, Aug 2016.

[55] J. Zhao, F. Zhai, J. Cheng, Q. He, J. Luo, X. Yang, et al., "Evaluating the Significance of Viscoelasticity in Diagnosing Early-Stage Liver Fibrosis with Transient Elastography," PLoS One, vol. 12, p. e0170073, 2017.

[56] J. A. Jensen, "A model for the propagation and scattering of ultrasound in tissue," $J$ Acoust Soc Am, vol. 89, pp. 182-90, Jan 1991.

[57] W. Schroeder and K. Martin, The Visualization Toolkit: An Object-Oriented Approach to 3-D Graphics, 4th ed. ed. Colombia, 2006.

[58] S. A. Maas, B. J. Ellis, G. A. Ateshian, and J. A. Weiss, "FEBio: finite elements for biomechanics," J Biomech Eng, vol. 134, p. 011005, Jan 2012.

[59] H. Si and K. Gartner, "3D boundary recovery by constrained Delaunay tetrahedralization," International Journal for Numerical Methods in Engineering, vol. 85, pp. 1341-1364, Mar 182011.

[60] J. Dutta-Moscato, A. Solovyev, Q. Mi, T. Nishikawa, A. Soto-Gutierrez, I. J. Fox, et al., "A Multiscale Agent-Based in silico Model of Liver Fibrosis Progression," Front Bioeng Biotechnol, vol. 2, p. 18, 2014.

[61] S. A. Maas, B. J. Ellis, G. A. Ateshian, and J. A. Weiss, "FEBio: Finite Elements for Biomechanics," Journal of Biomechanical Engineering, vol. 134, pp. 01100510, 2012.

[62] J. A. Jensen, "A model for the propagation and scattering of ultrasound in tissue," The Journal of the Acoustical Society of America, vol. 89, pp. 182-190, 1991.

[63] W. Schroeder, K. Martin, and B. Lorensen, The visualization toolkit, 2nd ed. Upper Saddle River, NJ: Prentice Hall PTR, 1998.

[64] National_Library_of_Medicine. The Visible Human Project Getting the Data [Online].

[65] ScanIP, "+FE, +NURBS and +CAD Tutorial Guide," Version 5.1 ed., 2001.

[66] M. Piccinelli, A. Veneziani, D. A. Steinman, A. Remuzzi, and L. Antiga, "A framework for geometric analysis of vascular structures: application to cerebral aneurysms," IEEE Trans Med Imaging, vol. 28, pp. 1141-55, Aug 2009.

[67] D. Mahr, "THREE-DIMENSIONAL, IN-SILICO BREAST PHANTOM FOR MULTIMODALITY IMAGE SIMULATIONS," Master, Bioengineering, University of Illinois at Urbana-Champaign, Urbana-Champaign, 2009.

[68] D. T. Ramsay, J. C. Kent, R. A. Hartmann, and P. E. Hartmann, "Anatomy of the lactating human breast redefined with ultrasound imaging," Journal of Anatomy, vol. 206, pp. 525-534, Jun 2005.

[69] M. J. Aftosmis, M. J. Berger, and J. E. Melton, "Robust and Efficient Cartesian Mesh Generation for Component-Based Geometry," presented at the 35th AIAA Aerospace Sciences Meeting, Reno NV, 1997.

[70] F. A. Duck, Physical properties of tissue : a comprehensive reference book / Francis A. Duck. London San Diego: Academic, 1990. 
[71] S. Goenezen, J. F. Dord, Z. Sink, P. E. Barbone, J. Jiang, T. J. Hall, et al., "Linear and nonlinear elastic modulus imaging: an application to breast cancer diagnosis," IEEE Trans Med Imaging, vol. 31, pp. 1628-37, Aug 2012.

[72] A. A. Oberai, N. H. Gokhale, S. Goenezen, P. E. Barbone, T. J. Hall, A. M. Sommer, et al., "Linear and nonlinear elasticity imaging of soft tissue in vivo: demonstration of feasibility," Phys Med Biol, vol. 54, pp. 1191-207, Mar 72009.

[73] D. R. Veronda and R. A. Westmann, "Mechanical characterization of skin-Finite deformations," J Biomech, vol. 3, pp. 111-124, 1970.

[74] Y. C. Fung, Biomechanics : mechanical properties of living tissues, 2nd ed. New York: Springer-Verlag, 1993.

[75] J. Jiang and T. J. Hall, "A fast hybrid algorithm combining regularized motion tracking and predictive search for reducing the occurrence of large displacement errors," IEEE Trans Ultrason Ferroelectr Freq Control, vol. 58, pp. 730-6, Apr 2011.

[76] F. Kallel and J. Ophir, "A least-squares strain estimator for elastography," Ultrasonic Imaging, vol. 19, pp. 195-208, 1997.

[77] J. Jiang, T. J. Hall, and A. M. Sommer, "A novel performance descriptor for ultrasonic strain imaging: a preliminary study," IEEE Trans Ultrason Ferroelectr Freq Control, vol. 53, pp. 1088-102, Jun 2006.

[78] Y. Qiu, M. Sridhar, J. K. Tsou, K. K. Lindfors, and M. F. Insana, "Ultrasonic viscoelasticity imaging of nonpalpable breast tumors: preliminary results," Acad Radiol, vol. 15, pp. 1526-33, Dec 2008.

[79] M. Bayer, T. J. Hall, L. P. Neves, and A. A. Carneiro, "Two-dimensional simulations of displacement accumulation incorporating shear strain," Ultrason Imaging, vol. 36, pp. 55-73, Jan 2014.

[80] J. A. Jensen, "A Model for the Propagation and Scattering of Ultrasound in Tissue," Journal of the Acoustical Society of America, vol. 89, pp. 182-190, Jan 1991.

[81] S. A. Maas, B. J. Ellis, G. A. Ateshian, and J. A. Weiss, "FEBio: Finite Elements for Biomechanics," Journal of Biomechanical Engineering-Transactions of the Asme, vol. 134, Jan 2012.

[82] J. F. Jiang and T. J. Hall, "A coupled subsample displacement estimation method for ultrasound-based strain elastography," Physics in Medicine and Biology, vol. 60, pp. 8347-8364, Nov 72015.

[83] T. Deffieux, J. L. Gennisson, J. Bercoff, and M. Tanter, "On the effects of reflected waves in transient shear wave elastography," IEEE Trans Ultrason Ferroelectr Freq Control, vol. 58, pp. 2032-5, Oct 2011.

[84] Y. Wang, E. Helminen, and J. F. Jiang, "Building a virtual simulation platform for quasistatic breast ultrasound elastography using open source software: A preliminary investigation," Medical Physics, vol. 42, pp. 5453-5466, Sep 2015.

[85] J. Bercoff, Tanter, M, Fink, M., "Supersonic shear imaging: a new technique for soft tissue elasticity mapping," IEEE Trans Ultrason Ferroelectr Freq Control, vol. 51, pp. 396-409, Apr 2004.

[86] Y. C. Fung, Biomechanics : mechanical properties of living tissues, 2nd ed. New York: Springer-Verlag, 1993. 
[87] T. A. Krouskop, T. M. Wheeler, F. Kallel, B. S. Garra, and T. Hall, "Elastic moduli of breast and prostate tissues under compression," Ultrasonic Imaging, vol. 20, pp. 260-274, Oct 1998.

[88] Z. G. Wang, Y. Liu, G. Wang, and L. Z. Sun, "Elastography method for reconstruction of nonlinear breast tissue properties," Int J Biomed Imaging, vol. 2009, p. 406854, 2009.

[89] A. C. Sharma, M. S. Soo, G. E. Trahey, and K. R. Nightingale, "Acoustic radiation force impulse imaging of in vivo breast masses," in Ultrasonics Symposium, 2004 IEEE, 2004, pp. 728-731 Vol.1.

[90] F. A. Duck, Physical properties of tissue : a comprehensive reference book. CA, USA: Academic Press: San Diego, 1990.

[91] J. McLaughlin and D. Renzi, "Shear wave speed recovery in transient elastography and supersonic imaging using propagating fronts," Inverse Problems, vol. 22, pp. 681-706, Apr 2006.

[92] F. Kallel, C. D. Prihoda, and J. Ophir, "Contrast-transfer efficiency for continuously varying tissue moduli: Simulation and phantom validation," Ultrasound in Medicine and Biology, vol. 27, pp. 1115-1125, Aug 2001.

[93] B. Peng, Y. Wang, W. Yang, T. Varghese, and J. Jiang, "Relative Elastic Modulus Imaging Using Sector Ultrasound Data for Abdominal Applications: An Evaluation of Strategies and Feasibility," IEEE Transactions on Ultrasonics, Ferroelectrics, and Frequency Control, vol. 63, pp. 1432-1440, 2016.

[94] P. N. T. Wells and H. D. Liang, "Medical ultrasound: imaging of soft tissue strain and elasticity," Journal of the Royal Society Interface, vol. 8, pp. 1521-1549, Nov 72011.

[95] J. R. Doherty, G. E. Trahey, K. R. Nightingale, and M. L. Palmeri, "Acoustic radiation force elasticity imaging in diagnostic ultrasound," IEEE Trans Ultrason Ferroelectr Freq Control, vol. 60, pp. 685-701, Apr 2013.

[96] E. T. Jaynes, "Information theory and statistical mechanics," Phys. Rev., vol. 106, pp. 620-630, 1957.

[97] D. P. Bertsekas, Nonlinear programming. Belmont, Mass.: Athena Scientific, 1995.

[98] G. Casella and R. L. Berger, Statistical inference. Pacific Grove, Calif:: Brooks/Cole Pub. Co., 1990.

[99] V. S. Riaben'kiı̌ and S. V. Tsynkov, A theoretical introduction to numerical analysis. Boca Raton, FL: Chapman \& Hall/CRC, 2007.

[100] H. Akaike, "Information theory and an extension of the maximum likelihood principle," 2nd International Symposium on Information Theory, 1973.

[101] D. F. Findley, "Counterexamples to Parsimony and Bic," Annals of the Institute of Statistical Mathematics, vol. 43, pp. 505-514, Sep 1991.

[102] B. C. B. Treeby, and J. Jaros, "K-Wave User Manual," 2012.

[103] T. Deffieux, J. L. Gennisson, J. Bercoff, and M. Tanter, "On the Effects of Reflected Waves in Transient Shear Wave Elastography," Ieee Transactions on Ultrasonics Ferroelectrics and Frequency Control, vol. 58, pp. 2032-2035, Oct 2011. 
[104] R. A. Standish, E. Cholongitas, A. Dhillon, A. K. Burroughs, and A. P. Dhillon, "An appraisal of the histopathological assessment of liver fibrosis," Gut, vol. 55, pp. 569-78, Apr 2006.

[105] P. Bedossa and T. Poynard, "An algorithm for the grading of activity in chronic hepatitis C. The METAVIR Cooperative Study Group," Hepatology, vol. 24, pp. 289-93, Aug 1996.

[106] A. Stenvall, E. Larsson, S. Strand, and B. Jonsson, "A small-scale dosimetry model of the liver tissue," European Journal of Nuclear Medicine and Molecular Imaging, vol. 39, pp. S199-S199, Oct 2012.

[107] G. S. a. K. Zalata, Ishak versus METAVIR: Terminology, Convertibility and Correlation with Laboratory Changes in Chronic Hepatitis C, 2011.

[108] Y. Huang, W. B. de Boer, L. A. Adams, G. MacQuillan, E. Rossi, P. Rigby, et al., "Image analysis of liver collagen using sirius red is more accurate and correlates better with serum fibrosis markers than trichrome," Liver International, vol. 33, pp. 1249-1256, Sep 2013.

[109] B. K. Kim, S. U. Kim, G. H. Choi, W. K. Han, M. S. Park, E. H. Kim, et al., "'Normal" liver stiffness values differ between men and women: A prospective study for healthy living liver and kidney donors in a native Korean population," Journal of Gastroenterology and Hepatology, vol. 27, pp. 781-788, Apr 2012.

[110] T. Khamdaeng, J. Luo, J. Vappou, P. Terdtoon, and E. E. Konofagou, "Arterial stiffness identification of the human carotid artery using the stress-strain relationship in vivo," Ultrasonics, vol. 52, pp. 402-411, Mar 2012.

[111] Z.-M. H. Seeram Ramakrishna, Ganesh V Kumar, Andrew W Batchelor, Joerg Mayer, An Introduction to Biocomposites: Imperial College Press, 2004.

[112] S. Torquato, Random heterogeneous materials : microstructure and macroscopic properties. New York: Springer, 2002.

[113] A. H. Bowker and G. J. Lieberman, Engineering statistics, 2d ed. Englewood Cliffs, N.J.,: Prentice-Hall, 1972.

[114] I. Sporea, S. Bota, O. Gradinaru-Taşcău, R. Sirli, A. Popescu, and A. Jurchiş, "Which are the cut-off values of 2D-Shear Wave Elastography (2D-SWE) liver stiffness measurements predicting different stages of liver fibrosis, considering Transient Elastography (TE) as the reference method?," European Journal of Radiology, vol. 83, pp. e118-22, 2014.

[115] W. Verlinden, S. Bourgeois, P. Gigase, C. Thienpont, L. Vonghia, T. Vanwolleghem, et al., "Liver Fibrosis Evaluation Using Real-time Shear Wave Elastography in Hepatitis C-Monoinfected and Human Immunodeficiency Virus/Hepatitis C-Coinfected Patients," J Ultrasound Med, vol. 35, pp. 1299-308, Jun 2016.

[116] P. Lewicki, "Long Time Evolution of Wavefronts in Random Media," SIAM Journal on Applied Mathematics, vol. Vol. 54, 1994.

[117] X. C. Yi Zheng, Aiping Yao, Haoming Lin, Yuanyuan Shen, Ying Zhu, Minhua $\mathrm{Lu}$, Tianfu Wang and Siping Chen, Shear Wave Propagation in Soft Tissue and Ultrasound Vibrometry, Wave Propagation Theories and Applications, 2013.

[118] A. K. A. Vinay Kumar, Jon C. Aster, Robbins \& Cotran Pathologic Basis of Disease, 9 edition ed.: Saunders, 1974. 
[119] J. W. Feng, C. F. Li, S. Cen, and D. R. J. Owen, "Statistical reconstruction of twophase random media," Computers \& Structures, vol. 137, pp. 78-92, Jun 2014.

[120] J. Y. Jeong, T. Y. Kim, J. H. Sohn, Y. Kim, W. K. Jeong, Y. H. Oh, et al., "Real time shear wave elastography in chronic liver diseases: accuracy for predicting liver fibrosis, in comparison with serum markers," World J Gastroenterol, vol. 20, pp. 13920-9, Oct 142014.

[121] J. K. Kang, J. Y. Cheong, S. W. Cho, J. H. Cho, J. S. Park, Y. B. Kim, et al., "[Liver stiffness measurement for the diagnosis of hepatic fibrosis in patients with chronic viral hepatitis]," Korean J Hepatol, vol. 13, pp. 521-9, Dec 2007.

[122] M. Lupsor-Platon, R. Badea, H. Stefanescu, Z. Sparchez, A. Serban, and A. Maniu, "The diagnosis performance of ultrasonic transient elastography for noninvasive assessment of liver fibrosis in 1138 chronic hepatitis $\mathrm{C}$ patients," 2011.

[123] R. Bataller and D. A. Brenner, "Hepatic stellate cells as a target for the treatment of liver fibrosis," Seminars in Liver Disease, vol. 21, pp. 437-451, Aug 2001.

[124] B. E. Treeby, J. Jaros, D. Rohrbach, and B. T. Cox, "Modelling Elastic Wave Propagation Using the k-Wave MATLAB Toolbox," IEEE International Ultrasonics Symposium Proceedings, pp. 146-149, 2014.

[125] Y. Wang, M. Wang, and J. Jiang, "An analysis of intrinsic variations of lowfrequency shear wave speed in a stochastic tissue model: the first application for staging liver fibrosis," Physics in Medicine and Biology, vol. 62, p. 1149, 2017.

[126] K. Nightingale, S. McAleavey, and G. Trahey, "Shear-wave generation using acoustic radiation force: In vivo and ex vivo results," Ultrasound in Medicine and Biology, vol. 29, pp. 1715-1723, Dec 2003.

[127] K. R. Nightingale, N. C. Rouze, S. J. Rosenzweig, M. H. Wang, M. F. Abdelmalek, C. D. Guy, et al., "Derivation and analysis of viscoelastic properties in human liver: impact of frequency on fibrosis and steatosis staging," IEEE Transactions on Ultrasonics, Ferroelectrics, and Frequency Control, vol. 62, pp. 165-175, 2015.

[128] S. Chen, W. Sanchez, M. R. Callstrom, B. Gorman, J. T. Lewis, S. O. Sanderson, et al., "Assessment of Liver Viscoelasticity by Using Shear Waves Induced by Ultrasound Radiation Force," Radiology, vol. 266, pp. 964-970, 2013.

[129] T. Deffieux, J. L. Gennisson, L. Bousquet, M. Corouge, S. Cosconea, D. Amroun, et al., "Investigating liver stiffness and viscosity for fibrosis, steatosis and activity staging using shear wave elastography," Journal of Hepatology, vol. 62, pp. 317324, Feb 2015.

[130] C. T. Barry, Z. Hah, A. Partin, R. A. Mooney, K.-H. Chuang, A. Augustine, et al., "Mouse Liver Dispersion for the Diagnosis of Early-Stage Fatty Liver Disease: A 70-Sample Study," Ultrasound in Medicine \& Biology, vol. 40, pp. 704-713, 4// 2014.

[131] Y. Wang, B. Peng, and J. Jiang, "Building an Open-source Simulation Platform of Acoustic Radiation Force-based Breast Elastography," Physics in Medicine and Biology, vol. In Press, p. XX, 2017.

[132] A. Solovyev, M. Mikheev, L. Zhou, J. Dutta-Moscato, C. Ziraldo, G. An, et al., "SPARK: A Framework for Multi-Scale Agent-Based Biomedical Modeling," 
International journal of agent technologies and systems, vol. 2, p. 10.4018/jats.2010070102, 2010.

[133] S. G. Hubscher, "Histological assessment of non-alcoholic fatty liver disease," Histopathology, vol. 49, pp. 450-65, Nov 2006.

[134] J. Schleicher, R. Guthke, U. Dahmen, O. Dirsch, H. G. Holzhuetter, and S. Schuster, "A theoretical study of lipid accumulation in the liver-implications for nonalcoholic fatty liver disease," Biochim Biophys Acta, vol. 1841, pp. 62-9, Jan 2014.

[135] A. M. Diehl, Z. P. Li, H. Z. Lin, and S. Q. Yang, "Cytokines and the pathogenesis of non-alcoholic steatohepatitis," Gut, vol. 54, pp. 303-6, Feb 2005.

[136] G. A. Pavliotis and A. Stuart, Multiscale Methods: Averaging and Homogenization vol. 53. Springer-Verlag New York., 2008.

[137] A. Divoux, J. Tordjman, D. Lacasa, N. Veyrie, D. Hugol, A. Aissat, et al., "Fibrosis in Human Adipose Tissue: Composition, Distribution, and Link With Lipid Metabolism and Fat Mass Loss," Diabetes, vol. 59, pp. 2817-2825, 2010.

[138] B. Boozari, A. Potthoff, I. Mederacke, A. Hahn, A. Reising, K. Rifai, et al., "Evaluation of sound speed for detection of liver fibrosis: prospective comparison with transient dynamic elastography and histology," J Ultrasound Med, vol. 29, pp. 1581-8, Nov 2010.

[139] Y. Wang, M. Wang, and J. J. Jiang, "An Analysis of Intrinsic Variations of Lowfrequency Shear Wave Speed in A Stochastic Tissue Model: The First Application for Staging Liver Fibrosis," Physics in Medicine and Biology, 2016.

[140] S. H. Chen, C. Y. Peng, I. P. Chiang, H. C. Lai, C. J. Lee, W. P. Su, et al., "Comparison of collagen proportionate areas in liver fibrosis quantification between chronic hepatitis B and C," Medicine, vol. 95, Aug 2016.

[141] A. P. Roberts, "Statistical reconstruction of three-dimensional porous media from two-dimensional images," Physical Review E, vol. 56, pp. 3203-3212, Sep 1997.

[142] E. Nunez, E. W. Steyerberg, and J. Nunez, "[Regression modeling strategies]," Rev Esp Cardiol, vol. 64, pp. 501-7, Jun 2011.

[143] Jong Yoon Bahk, Zhengfu Piao, Jae Hun Jung, and H. Han, "Treatment of the end Stage Liver Cirrhosis by Human Umbilical Cord Blood Stem Cells: Preliminary Results," 2011.

[144] S. K. Sarin and A. Choudhury, "Acute-on-chronic liver failure: terminology, mechanisms and management," Nat Rev Gastroenterol Hepatol, vol. 13, pp. 13149, Mar 2016.

[145] L. A. Adams, P. Angulo, and K. D. Lindor, "Nonalcoholic fatty liver disease," CMAJ, vol. 172, pp. 899-905, Mar 292005.

[146] K. D. Fairbanks, "Alcoholic Liver Disease," Online CME, 2012.

[147] C. Liedtke, T. Luedde, T. Sauerbruch, D. Scholten, K. Streetz, F. Tacke, et al., "Experimental liver fibrosis research: update on animal models, legal issues and translational aspects," Fibrogenesis Tissue Repair, vol. 6, p. 19, Oct 012013.

[148] S. Ricard-Blum, S. Bresson-Hadni, D. A. Vuitton, G. Ville, and J. A. Grimaud, "Hydroxypyridinium collagen cross-links in human liver fibrosis: study of alveolar echinococcosis," Hepatology, vol. 15, pp. 599-602, Apr 1992. 
[149] S. Ghatak, E. V. Maytin, J. A. Mack, V. C. Hascall, I. Atanelishvili, R. Moreno Rodriguez, et al., "Roles of Proteoglycans and Glycosaminoglycans in Wound Healing and Fibrosis," Int J Cell Biol, vol. 2015, p. 834893, 2015.

[150] L. Rubbia-Brandt, P. Fabris, S. Paganin, G. Leandro, P. J. Male, E. Giostra, et al., "Steatosis affects chronic hepatitis C progression in a genotype specific way," Gut, vol. 53, pp. 406-12, Mar 2004.

[151] X. Chen, K. Xun, L. Chen, and Y. Wang, "TNF- $\alpha$, a potent lipid metabolism regulator," Cell Biochemistry and Function, vol. 27, pp. 407-416, 2009.

[152] T. Glatard, C. Lartizien, B. Gibaud, R. F. da Silva, G. Forestier, F. Cervenansky, et al., "A virtual imaging platform for multi-modality medical image simulation," IEEE Trans Med Imaging, vol. 32, pp. 110-8, Jan 2013.

[153] L. Antiga, B. Ene-Iordache, and A. Remuzzi, "Computational geometry for patient-specific reconstruction and meshing of blood vessels from MR and CT angiography," IEEE Trans Med Imaging, vol. 22, pp. 674-84, May 2003.

[154] T. A. Krouskop, T. M. Wheeler, F. Kallel, and T. Hall, "The elastic moduli of breast and prostate tissues under compression," Ultrasonic Imaging, vol. 20, pp. 260-274, 1998.

[155] P. S. Wellman, E. P. Dalton, D. Krag, K. A. Kern, and R. D. Howe, "Tactile imaging of breast masses: first clinical report," Arch Surg, vol. 136, pp. 204-8, Feb 2001.

[156] R. J. Lapeer, P. D. Gasson, and V. Karri, "A hyperelastic finite-element model of human skin for interactive real-time surgical simulation," IEEE Trans Biomed Eng, vol. 58, pp. 1013-22, Apr 2011.

[157] M. A. Puso and J. A. Weiss, "Finite element implementation of anisotropic quasilinear viscoelasticity using a discrete spectrum approximation," J Biomech Eng, vol. 120, pp. 62-70, Feb 1998.

[158] A. I. Beltzer and T. Sato, "Probability distribution of wave velocity in heterogeneous media due to random phase configuration," Wave Motion, vol. 38, pp. 221-227, Sep 2003.

[159] B. A. Ogunnaike, Random Phenomena: Fundamentals of Probability and Statistics for Engineers. CRC Press: Taylor \& Francis, 2009.

[160] D. J. C. MacKay, Information theory, inference, and learning algorithms. Cambridge, UK ; New York: Cambridge University Press, 2003.

[161] T. M. Cover and J. A. Thomas, Elements of information theory. New York: Wiley, 1991.

[162] J. J. Bradley E. Treeby, Daniel Rohrbach, B. T. Cox, "Modelling Elastic Wave Propagation Using the k-Wave MATLAB Toolbox," IEEE International Ultrasonics Symposium Proceedings, 2014.

[163] Q. H. Liu, "Large-scale simulations of electromagnetic and acoustic measurements using the pseudospectral time-domain (PSTD) algorithm," Ieee Transactions on Geoscience and Remote Sensing, vol. 37, pp. 917-926, Mar 1999.

[164] M. Caputo, J. M. Carcione, and F. Cavallini, "Wave simulation in biologic media based on the Kelvin-Voigt fractional-derivative stress-strain relation," Ultrasound Med Biol, vol. 37, pp. 996-1004, Jun 2011. 
[165] J. K. Reddy and M. S. Rao, "Lipid metabolism and liver inflammation. II. Fatty liver disease and fatty acid oxidation," Am J Physiol Gastrointest Liver Physiol, vol. 290, pp. G852-8, May 2006. 


\section{Appendix A: Geometry Creation}

A description of the creation of randomly distributed structures is provided below for the sake of completeness. These steps were implemented in VTK using standard C++ language. Python scripts derived from Vascular Modeling ToolKit (VMTK) [153] were used to call $\mathrm{C}++$ programs to execute. The detailed procedures related to creating milk ducts including lobes are shown below as a three-step process.

Step 1: Creation of random milk duct

Starting from two random points (i.e. the first line segment of a random curve) within the fibro-glandular region, subsequent points were generated by adding more line segments to continue this random curve. Subsequent directions of this curve were determined by a random vector. This random vector largely followed the direction of the two most recent points on the curve, with a small added fraction (e.g. 20\%) of randomness. All points were tested to ensure the curve had been constrained within the fibro-glandular region.

If a pre-determined length of the random tube was reached or the random curve had to leave the fibro-glandular region, the process of generating random points would stop. The random curve was the skeleton for the simulated milk duct. The diameter of the tube can spatially vary as specified by the user. Finally, a milk duct structure was linked together smoothly by VTK using vtkTubeFilter. An example of the simulated milk duct is given in Fig. A1-(a) below. 


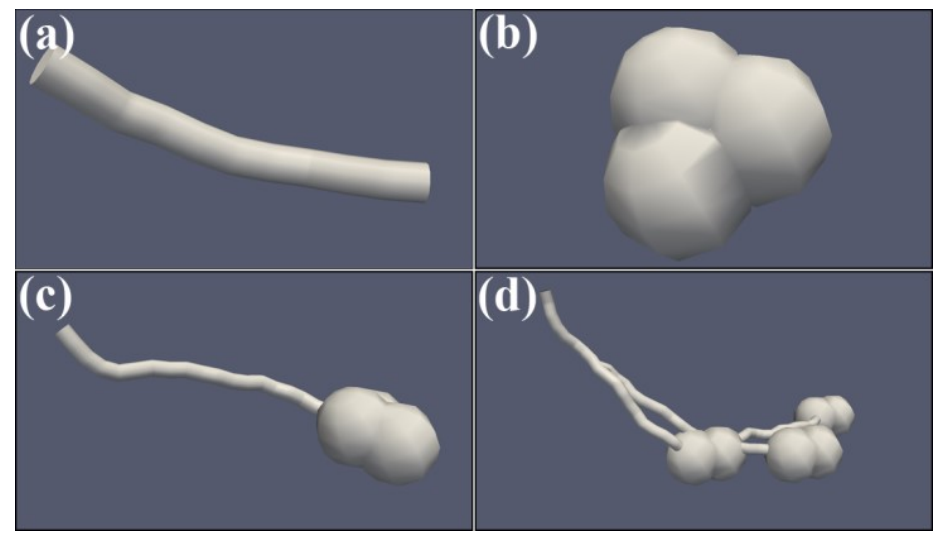

Figure A1. An example of generating random milk duct and lobe structure using VTK: (a) A milk duct represented by a random tube; (b) a lobe structure represented by a cluster of overlapping spheres; (c) one milk duct connected with a lobe; and (d) several simulated branches of milk ducts and lobes.

\section{Step 2: Creation of simulated lobes}

A simulated lobe was represented by a cluster of overlapping random spheres as shown in Fig. A1b. Typically, a simulated lobe consisted of 3-5 random spheres and occupied 4-9 $\mathrm{mm}^{3}$ in space. Of note, overlapping random spheres were stitched together using Union Operations under VTK.

\section{Step 3: Integration of branches}

Two random points on the random curve (as described in Step 1) could be selected as the starting point to create bi-furcated milk duct structures. Once those two new starting points were selected, the process of generating one new milk duct was the same as what described in Step 1. The new branch of the milk duct was connected to the previously generated milk duct through the same Union operation under VTK. Similarly, all simulated lobes were connected to milk ducts through the same Union operation (see Fig. A1c). Finally, all these branches were linked together to create a tree-like structure to represent milk duct and lobe structure (see Fig. A1d). 
Creation of the small adipose compartments (elongated random ellipsoids) within the fibro-glandular region was similar to what was described in Step 2 for the generation of simulated breast lobes, except that the small fat compartments were represented as a small cluster of random ellipsoids. Similarly, generating Cooper's ligaments was similar to what was described in Step 1 above. 


\section{Appendix B: Tissue Mechanical Models Used for QUE Simulations}

For FE-simulations of QUE, a hyper-elasticity model, Veronda-Westmann (V-W) model [73], was adopted. In the V-W model, the strain energy function is given as follows,

$$
W=C_{1}\left[e^{\left(C_{2}\left(I_{1}-3\right)\right)}-1\right]-\frac{C_{1} C_{2}}{2}\left(I_{2}-3\right)+U(J)
$$

where $C_{1}$ and $C_{2}$ are, respectively, the first and second Veronda-Westmann coefficients, $I_{1}$ and $I_{2}$ are, respectively, the first and second invariant of the right Cauchy-Green tensor. Incompressibility requires the volumetric strain function $\mathrm{U}(\mathrm{J})=0$.

Uniaxial stress-strain curves of glandular tissue, fat, skin, fibroadenoma and invasive ductal carcinoma were fit to the $\mathrm{V}-\mathrm{W}$ model. Those stress-strain curves were derived based on previously published results. [154-156] To our knowledge stress-strain curve of Cooper's ligament is not available in the literature. Material properties of the Cooper's ligament were derived from other ligament data in the literature.

Table B1. Parameters associated with the V-W model

\begin{tabular}{llll}
\hline \hline Tissue Type & $C_{1}(\mathbf{k P a})$ & $C_{2}$ \\
\hline Fat & 3 & 5 & \\
$\begin{array}{l}\text { Fibro-glandular } \\
\text { tissue }\end{array}$ & 4 & 6.5 & \\
$\begin{array}{l}\text { Cooper's } \\
\text { ligament }\end{array}$ & 25 & 7 \\
Milk duct & 3 & 2 \\
Skin & 300 & 5 & \\
Benign lesion & 30 & 7
\end{tabular}


In the time-dependent QUE application, viscoelasticity of soft tissues was taken into consideration by a quasi-linear viscoelastic material model[74] implemented in FEBio[61]. In the quasi-linear viscoelastic model, a relaxation function $G(t)$ is used[157] as follows,

$$
G(t)=1+\sum_{i=1}^{N} \gamma_{i} \exp \left(-t / \tau_{i}\right)
$$

where $\tau_{i}$ are one value representing relaxation time, and $\gamma_{i}$ is one coefficient. In this study, $\mathrm{N}$ was set to 1 and Eqn. (B2) became the classic Kevin-Voigt model. Viscoelastic parameters used in the time-dependent QUE application are shown in Table B2 below.

Table B2. Viscoelastic coefficients and relaxation times used in the FEA simulations

\begin{tabular}{llll}
\hline Component & $\begin{array}{l}\text { Viscoelastic coefficient } \\
\left(\gamma_{1}\right)\end{array}$ & $\begin{array}{l}\text { Relaxation } \\
\text { time of Case 1 } \\
\left(\tau_{\mathbf{1}}\right) \text { Unit: } \\
\text { second }\end{array}$ & $\begin{array}{l}\text { Relaxation } \\
\text { time of Case 2 } \\
\left(\tau_{\mathbf{1}}\right) \text { Unit: } \\
\text { second }\end{array}$ \\
\hline Fat & 0.95 & 1 & 1 \\
$\begin{array}{l}\text { Fibro-glandular } \\
\text { tissue }\end{array}$ & 0.95 & 1.2 & 3 \\
$\begin{array}{l}\text { Cooper's } \\
\text { ligament }\end{array}$ & 0.95 & 1.5 & 1.5 \\
$\begin{array}{l}\text { Milk duct } \\
\text { Skin }\end{array}$ & 0.95 & 1 & 1 \\
Lesion & 0.95 & 1 & 1 \\
\hline \hline
\end{tabular}




\section{Appendix C: Derivation of PDF for SWS Distribution in Random Multiphase Media}

Let's consider an elastic heterogeneous medium, which consists of multiple isotropic phases with elastic bulk modulus $\left(K_{i}\right)$, shear modulus $\left(G_{i}\right)$, mass density $\left(\rho_{i}\right)$, and the volume concentration $\left(c_{i}, \sum c_{i}=1\right)$, respectively. The subscript $i$ denotes those phase indices, respectively. For simplicity, the multi-phase medium is treated as effectively isotropic and macroscopically homogeneous, which is characterized by effective bulk modulus $(K)$, effective shear modulus $(G)$ and effective density $\left(\rho, \rho=\sum \rho_{i} c_{i}\right)$ [158]. Without the loss of generality, a set of physical properties $J=\left(K_{i}, G_{i}, \rho_{i}, c_{i}\right)$ is defined for this random multi-phase medium. In this derivation, both $K$ and $G$ are random variables.

The macroscopic SWS $\left(V_{S}\right)$ of this elastic random medium can be related to the effective shear modulus $G$ by [94],

$$
V_{S}=\sqrt{\frac{G}{\rho}}
$$

where $\rho$ is effective density.

Recall that both $G$ and $V_{s}$ are random variables. We define $F\left(V_{S}\right)$ as a cumulative distribution function (CDF) of $V_{s}$. By definition, $F\left(V_{s}\right)$ can be written as follows [159],

$$
F\left(V_{s}\right)=F\left\{v \geq V_{s}\right\}=F\left\{\sqrt{\frac{G}{\rho}} \geq V_{s}\right\}=F\left\{G \geq \rho \cdot V_{s}^{2}\right\}=\int_{0}^{\rho \cdot V_{s}^{2}} p(G) d_{G}
$$

where $p(G)$ is the probability density function (PDF) of effective shear modulus $G$. Hence, the PDF of $V_{s}$ can be written as,

$$
p\left(V_{S}\right)=\frac{\partial F\left(V_{S}\right)}{\partial v}=\frac{\partial \int_{0}^{\rho \cdot V_{S}^{2}} p(G) d_{G}}{\partial v}=p\left(\rho \cdot V_{s}^{2}\right) \cdot 2 \rho V_{S}
$$

Now we can establish a link between $p\left(V_{s}\right)$ and $p(G)$ as follows, 


$$
p\left(V_{s}\right)=p(G) 2 V_{s} \rho
$$

The Jaynes' principle of maximum entropy states that the information entropy $(H)$ is given by [96],

$$
H=-\int_{-\infty}^{+\infty} p(G) \ln \frac{p(G)}{m(G)} d G
$$

where $m(G)$ is an initial distribution of data and is typically assigned according to a prior understanding of data $G$. The Jaynes' entropy $H$ can reach maximum if $p(G)$ can reflect the data distribution in the best possible way. Consequently, maximization of $H$ leads to an optimized $p(G)$ that best describes the given data $G$.

$m(G)$ was assigned to be a uniform distribution in the current study so that no bias was injected. Consequently, Eqn. (C5) is equivalent to the classical Shannon's entropy given by

$$
H=-\int_{-\infty}^{+\infty} p(G) \ln p(G) d G
$$

Now we maximize $H$ that are subjected to three constraints, i.e. the zero ${ }^{\text {th }}$, first and second order moments as follows,

$$
\begin{aligned}
& \int_{-\infty}^{+\infty} p(G) d G=1 \\
& \int_{-\infty}^{+\infty} p(G) G d G=G_{E} \text { and } \\
& \int_{-\infty}^{+\infty} p(G)\left(G-G_{E}\right)^{2} d G=G_{D}
\end{aligned}
$$

To maximize H, the class Lagrange Multiplier method [97] was used and the Lagrange function was constructed as follows,

$$
\begin{aligned}
& L(p, \lambda)=-\int_{-\infty}^{+\infty} p(G) \ln p(G) d G+\lambda_{0}( \\
& \int_{-\infty}^{+\infty} p(G) d G-
\end{aligned}
$$




$$
\begin{aligned}
& 1)+\lambda_{1}\left(\int_{-\infty}^{+\infty} p(G) G d G-G_{E}\right) \\
& +\lambda_{2}\left(\int_{-\infty}^{+\infty} p(G)\left(G-G_{E}\right)^{2} d G-G_{D}\right)
\end{aligned}
$$

where $\lambda_{0}, \lambda_{1}$ and $\lambda_{2}$ are Lagrange multipliers.

$\mathrm{L}(\mathrm{p}, \lambda)$ reaches the maximum when $\frac{\partial L(p, L)}{\partial p(G)}=0$. The derivative of $L(p, \lambda)$ with respect to $p(G)$ can be expressed as $[160,161]$

$$
\frac{\partial L(p, L)}{\partial p(G)}=-\ln p(G)+\lambda_{0}+\lambda_{1}+\lambda_{2}\left(G-G_{E}\right)^{2}
$$

Solving Eqn. (A9), we have

$$
p(G)=e^{\lambda_{0}+\lambda_{1} G+\lambda_{2}\left(G-G_{E}\right)^{2}}
$$

Substituting Eqn. (C10) into Eqn. (C7-a), we obtain following equations

$$
\int_{-\infty}^{+\infty} e^{\lambda_{0}+\lambda_{1} G+\lambda_{2}\left(G-G_{E}\right)^{2}} d G=1
$$

which provides

$$
e^{\lambda_{0}} \sqrt{\frac{\pi}{-\lambda_{2}}} \int_{-\infty}^{+\infty} e^{\lambda_{1} G} f(G) d G=1
$$

where $f(G)$ is the Gaussian distribution with mean $G_{E}$ and variance $\sigma^{2}$ with $\sigma=\sqrt{\frac{-1}{2 \lambda_{2}}}$

According to the moment-generating function of a Gaussian random variable, Eqn. (C11-b) can be rewritten as follows,

$$
e^{\lambda_{0}} \sqrt{\frac{\pi}{-\lambda_{2}}} e^{G_{E} \lambda_{1}+\frac{\sigma^{2} \lambda_{1}^{2}}{2}}=1
$$

Similarly, replacing $p(G)$ in Eqn. (C7-b) with Eqn. (C10), we can also obtain following equation

$$
\int_{-\infty}^{+\infty} e^{\lambda_{0}+\lambda_{1} G+\lambda_{2}\left(G-G_{E}\right)^{2}} G d G=G_{E}
$$


Some algebra shows that Eqn. (C13-a) becomes

$$
e^{\lambda_{0}+\lambda_{2} G_{E}{ }^{2}-\lambda_{2}\left(G_{E}-\frac{\lambda_{1}}{2 \lambda_{2}}\right)^{2}} \sqrt{2 \pi} \sigma E(G)=G_{E}
$$

where $E(G)=G_{E}-\frac{\lambda_{1}}{2 \lambda_{2}}$. By using Eqn. (C13-b) over Eqn. (C12), we can obtain

$$
\frac{e^{\lambda_{0}+\lambda_{2} G_{E}{ }^{2}-\lambda_{2}\left(G_{E}-\frac{\lambda_{1}}{2 \lambda_{2}}\right)^{2}} \sqrt{2 \pi} \sigma E(G)}{e^{\lambda_{0}} \sqrt{\frac{\pi}{-\lambda_{2}}} e^{G_{E} \lambda_{1}+\frac{\sigma^{2} \lambda_{1}{ }^{2}}{2}}}=\frac{G_{E}}{1}
$$

By simplifying Eqn. (C14-a), we obtain

$$
\begin{aligned}
& G_{E}-\frac{\lambda_{1}}{2 \lambda_{2}}=G_{E}, \text { which gives } \\
& \lambda_{1}=0
\end{aligned}
$$

By plugging $\lambda_{1}=0$ into Eqn. (C11-b), we obtain $\lambda_{0}=\ln \sqrt{\frac{\lambda_{2}}{-\pi}}$.

Replacing $p(G)$ in Eqn. (C7-c) with Eqn. (C10), we obtain following equations,

$$
e^{\lambda_{0}} \int_{-\infty}^{+\infty} \omega^{2} e^{\lambda_{2} \omega^{2}} d \omega=G_{D}, \omega=G-G_{E}
$$

which shows that $\lambda_{2}=\frac{-1}{2 G_{D}}$

Thus, Eqn. (C10) becomes a form of the Gaussian distribution given by

$$
p(G)=\frac{1}{\sqrt{2 \pi G_{D}}} e^{\frac{-\left(G-G_{E}\right)^{2}}{2 G_{D}}}
$$

where $\lambda_{0}=\ln \frac{1}{\sqrt{2 \pi G_{D}}}, \lambda_{1}=0$, and $\lambda_{2}=\frac{-1}{2 G_{D}}$.

Now, we can substitute Eqn. (C1) and (C13) into Eqn. (C4). The final form of the PDF of the SWS $p\left(V_{s}\right)$ is given as follows,

$$
p\left(V_{S}\right)=\frac{1}{\sqrt{2 \pi G_{D}}} e^{\frac{-\left(V_{S}^{2} \rho-G_{E}\right)^{2}}{2 G_{D}}} 2 \rho V_{S}
$$




\section{Appendix D: A Brief Description of K-Wave Simulations}

The elastic shear wave propagation was simulated using an open-source wave simulator K-Wave [162]. In a simple form, the elastic wave propagation in an isotropic linearly elastic medium is governed by two coupled first-order partial differential equations (PDEs) [162]:

$$
\begin{aligned}
& \frac{\partial \sigma_{i j}}{\partial t}=\lambda \delta_{i j} \frac{\partial v_{k}}{\partial x_{k}}+\mu\left(\frac{\partial v_{i}}{\partial x_{j}}+\frac{\partial v_{j}}{\partial x_{i}}\right) \\
& \frac{\partial v_{i}}{\partial t}=\frac{1}{\rho_{0}} \frac{\partial \sigma_{i j}}{\partial x_{j}}
\end{aligned}
$$

where $v, x, \sigma, t$ and $\rho_{0}, \delta_{i j}$ are the particle velocity, the coordinate system, stress tensor, time, mass density and Kronecker delta, respectively. In Eqn. (D1), $\lambda$ and $\mu$ are the Lame parameters. All subscripts follow Einstein notation in this Appendix.

In this study, Eqns. (D1-D2) were numerically solved by Fourier pseudo-spectral method $[163,164]$ in K-WAVE on a $2 \mathrm{D}$ rectilinear grid $\left[0, L_{x}\right] \times\left[0, L_{y}\right]$. A graphical illustration of the computing domain is given in Figure B1. A transient elastography-like excitation was applied onto a vertical line $x_{1}=L_{x e}$ (see Figure B1) as an interior boundary condition,

$$
\sigma_{2}\left(L_{x e}, x_{2}, t\right)=\left\{\begin{array}{c}
k \sin 2 \pi f t, 0 \leq x_{2} \leq L_{y}, 0 \leq t \leq \frac{1}{f} \\
0,0 \leq x_{2} \leq L_{y}, t \geq \frac{1}{f}
\end{array}\right.
$$

where $f$ is the excitation frequency, $k$ is an arbitrary constant and $t$ is the time. Basically, this interior boundary states that one cycle of a perturbation $\left(\sigma_{2}\right)$ is prescribed near the ROI to induce plane shear wave propagating along the $x_{1}$ direction. 
The well-known Dirichlet periodic boundary condition was applied onto four exterior edges (see Lines 1-4 in Fig. D1) to create a "mirroring" structure around all four edges. Loosely speaking, under this periodic boundary condition, the SW wave-front can re-appear at Line 4 when the SW wave-front passed Line 2. Mathematically, this exterior periodic boundary condition can be written as follows:

$$
\begin{aligned}
& \sigma_{i j}\left(0, x_{2}, t\right)=\sigma_{i j}\left(L_{x}, x_{2}, t\right), 0 \leq x_{2} \leq L_{y} \\
& \sigma_{i j}\left(x_{1}, 0, t\right)=\sigma\left(x_{1}, L_{y}, t\right), 0 \leq x_{1} \leq L_{x} \\
& v_{i}\left(0, x_{2}, t\right)=v_{i}\left(L_{x}, x_{2}, t\right), 0 \leq x_{2} \leq L_{y} \\
& v_{i}\left(x_{1}, 0, t\right)=v_{i}\left(x_{1}, L_{y}, t\right), 0 \leq x_{1} \leq L_{x}
\end{aligned}
$$

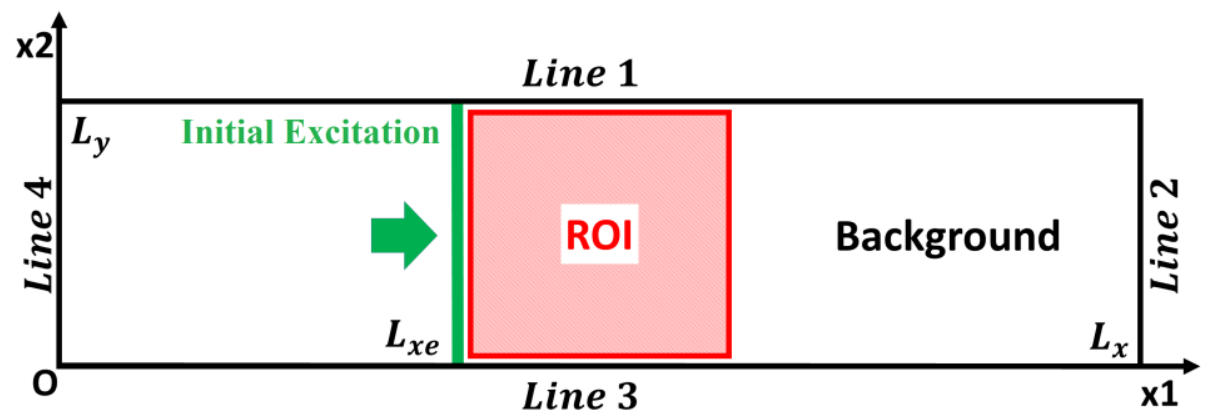

Figure D1. A graphical illustration of the boundary conditions defined for K-Wave plane SW simulation presented in the Materials and Methods Section. The computing area is defined on a rectilinear grid $\left[0, L_{x}\right] \times\left[0, L_{y}\right]$. The green arrow indicates the SW propagation direction.

In this study, the above-mentioned periodic boundary condition was used, as opposed to the perfectly matched layer (PML) for two reasons. First, the PML boundary condition resulted in apparent constraints (i.e. absorption) around the top and bottom boundaries (particularly, Lines 1 and 3 in Figure D1). That would be a violation of the plane shear wave assumption. Second, the absorption of the PML in K-WAVE was mainly 
designed for high-frequency compression waves (in $\mathrm{MHz}$ ) and therefore, was not well fitted for low-frequency $(50-200 \mathrm{~Hz}) \mathrm{SW}$ in terms of its effect of wave absorption. 


\section{Appendix E: A Description of Systems Biology Simulations}

As shown in Fig. E1, a brief step-by-step description of the proposed systems biology (SB) simulations using SPARK is provided below for the sake of completeness. The relevant codes can be downloaded from Github (https://github.com/jjiang-mtu).

Figure E1. A flowchart illustrating the execution of systems biology simulations.

\section{Initialization}

At the beginning, agents, system variables, and simulation space were set to initial values as suggested from the literature (see the source codes). At the initialization step (Fig. E2a), hepatocytes were arranged in lobules and each lobule was modeled as a hexagon. In the current setting, each edge of those hexagons was approximately $1 \mathrm{~mm}$ based on pathology [106]; each septum, forming an edge of a lobule, was connected by portal triads. Major anatomical features included rules (i.e. five major schemes described in the 
Materials Section) and parameters related to chemical and biological agents were also initialized in this step.

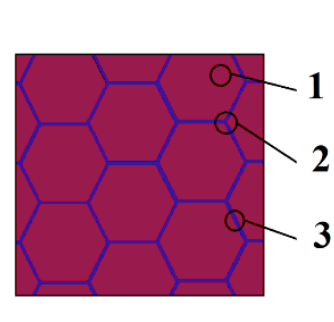

(a)

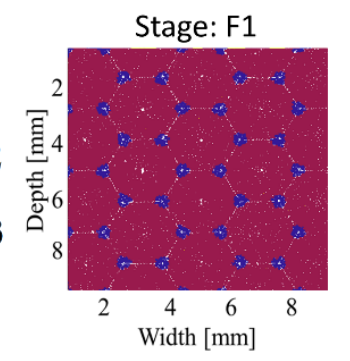

(b)

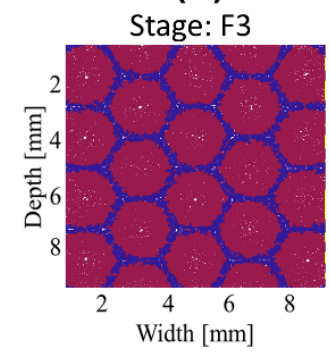

(d)

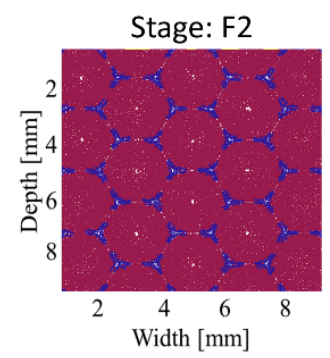

(c)

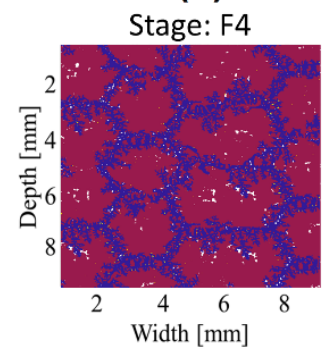

(e)

Figure E2. Schematic illustrations of the systems biology-based tissue model: (a) Initial setup; (b) Stage F1 with 7.8\% collagenous tissue; (c) Stage F2 with $9.6 \%$ collagenous tissue; (d) Stage F3 with $23.3 \%$ collagenous tissues; and (e) Stage F4 with $34.5 \%$ collagenous tissues. In (a)-(e), the red is normal liver tissue and the blue is collagenous connective tissue. F1 to F4 were referred as to the METAVIR classifications [105].

\section{Execution of Systems Biology Simulations}

To simplify the description, we first assumed the liver fibrosis would be simulated alone. Based on the first four major schemes described in Fig. 5.2, liver injuries triggered depositions of collagen after the $\mathrm{CCL}_{4}$ had started to diffuse through the simulation space. Consequently, in the early stages, the collagen was deposited around portal tracts with extensions into adjacent peri-portal regions along septa, as shown in Fig. E2b-c and those collagen depositions looked like isolated islands (Fig. E2b-c). As the liver fibrosis progressed deaths of hepatocytes and activation of fibroblast gradually took place across 
the simulation space (i.e. a term defined in SPARK) and, fibrous expansions of most portal areas caused portal-to-portal bridging or portal-to-central bridging (Zalata, 2011), as shown in Fig. E2d-e. In short, at the late stages of the liver fibrosis, deposited collagen "islands" (see Fig. E2b-c) were connected by septa and the overall architectural appearance looked more like a complex web (see Fig. E2e). Overall, the proposed SB simulation resulted in a stochastic tissue model capturing both the increasing presence of collagen content and spatial characteristics of collagen depositions, as shown in Fig. E2b-e.

If the simulations of liver fibrosis and steatosis were executed together, in addition to the depositions of collagen content, lipids were simultaneously accumulated and the deposition of lipids was relatively distributed over the entire simulation space. Two examples are provided in Fig. E3. In Fig. E3a, the collagen was deposited around portal tracts at the beginning stage. The liver cells were filled with multiple lipid droplets. The hepatocytes look like small fat vacuoles. In the late stage (Fig. E3b), the size of the vacuoles increases and distributed over the entire space [165].

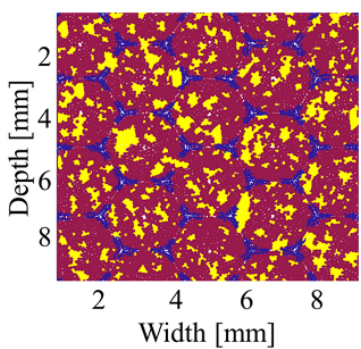

(a)

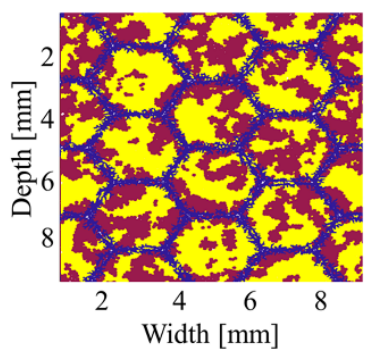

(b)

Figure E3. Histological characteristics of combined liver steatosis and fibrosis simulated by SPARK. The red, yellow and blue colors represent normal tissue, fat, and fibrosis, respectively. In (a), the CPA and the fat fractions were $7.8 \%$ and $22 \%$, respectively, while in (b), the CPA and fat fraction were $23.3 \%$ and $50 \%$, respectively. 


\section{Appendix F: Copyright Permission of Content in Chapter 2}

Content in Chapter 2 is reprinted from the following paper. A copy of the granted permission for reproduction is shown below.

1. Y. Wang, E. Helminen, and J. F. Jiang, "Building a virtual simulation platform for quasistatic breast ultrasound elastography using open source software: A preliminary investigation," Medical Physics, vol. 42, pp. 5453-5466, Sep 2015.

JOHN WILEY AND SONS LICENSE

TERMS AND CONDITIONS

Apr 05, 2017

This Agreement between yu wang ("You") and John Wiley and Sons ("John Wiley and Sons") consists of your license details and the terms and conditions provided by John Wiley and Sons and Copyright Clearance Center.

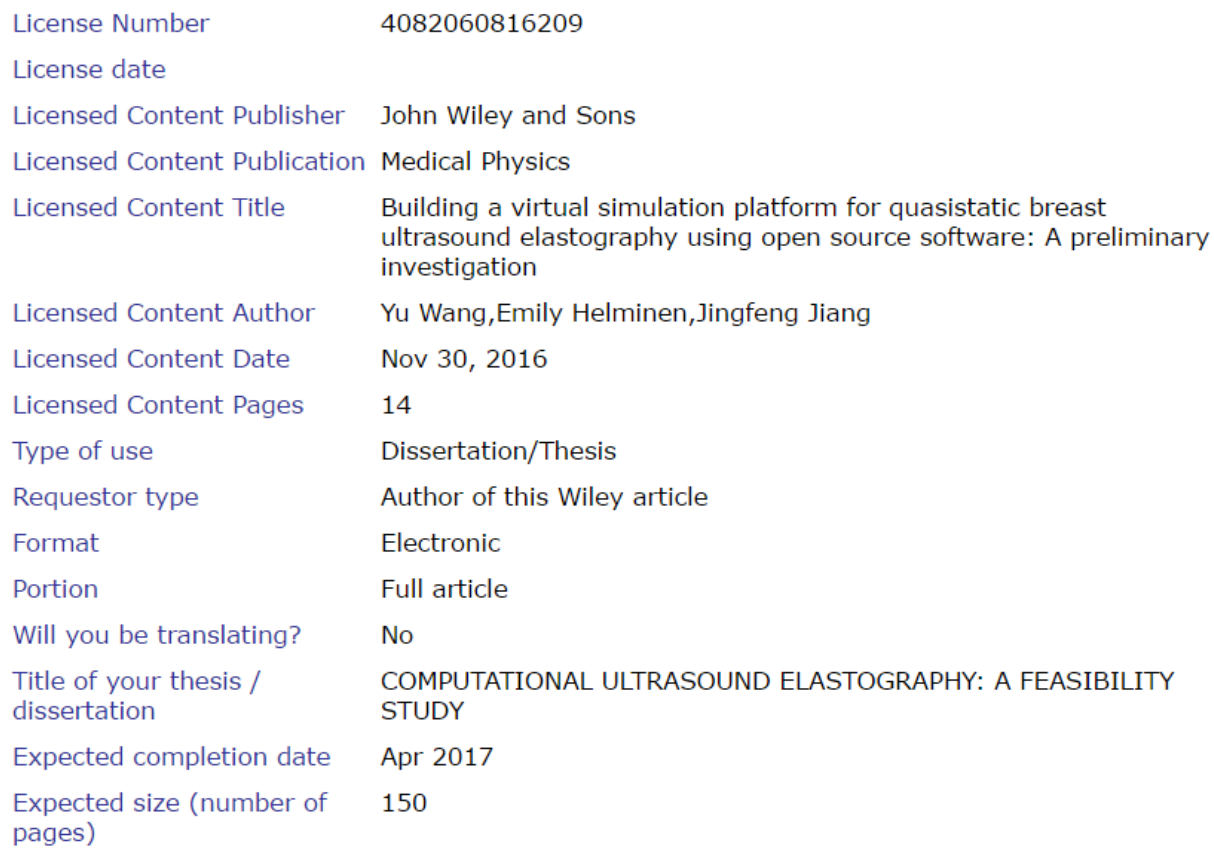




\section{Appendix G: Copyright Permission of Content in Chapter 3 and 4}

Content in Chapter 3 and 4 are reprinted from the following papers. A copy of the granted permission for reproduction is shown below.

1. Y. Wang, B. Peng, and J. Jiang, "Building an open-source simulation platform of acoustic radiation force-based breast elastography," Phys Med Biol, vol. 62, pp. 19491968, Mar 072017.

2. Y. Wang, M. Wang, and J. J. Jiang, "An Analysis of Intrinsic Variations of Lowfrequency Shear Wave Speed in A Stochastic Tissue Model: The First Application for Staging Liver Fibrosis," Physics in Medicine and Biology, 2016.

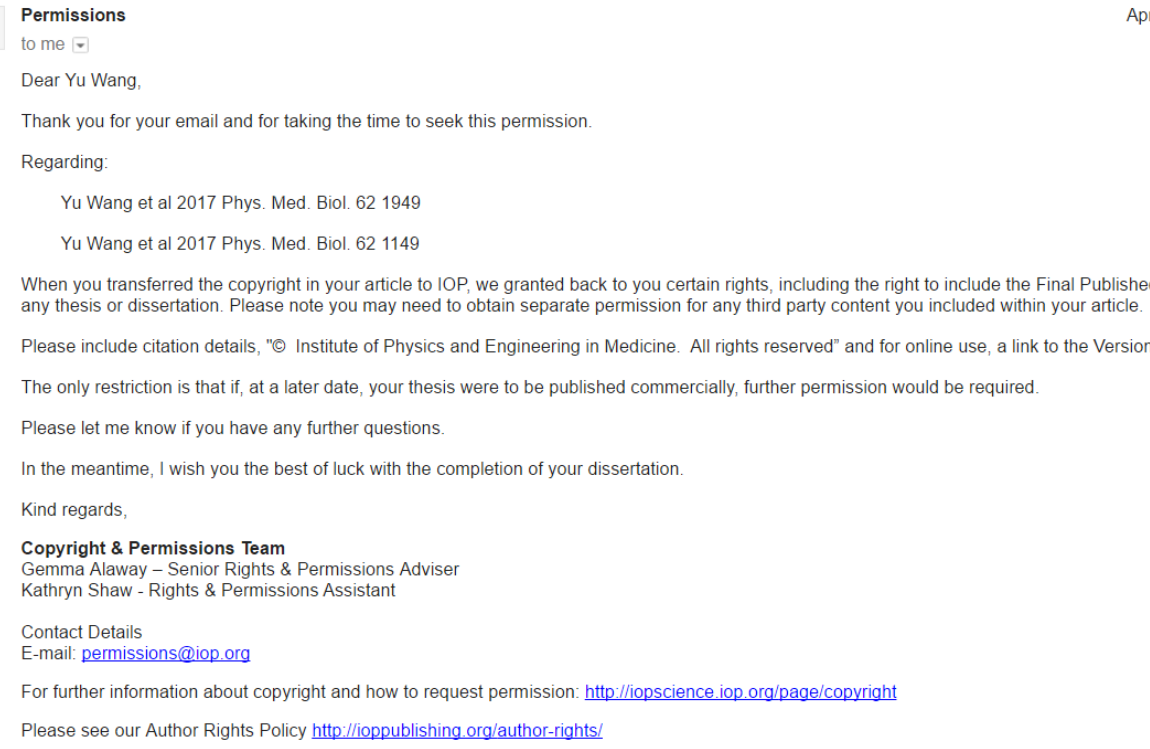

